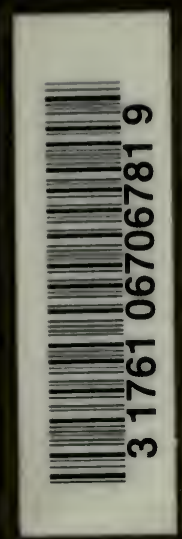

UWV of TORONTO LIBFARY 



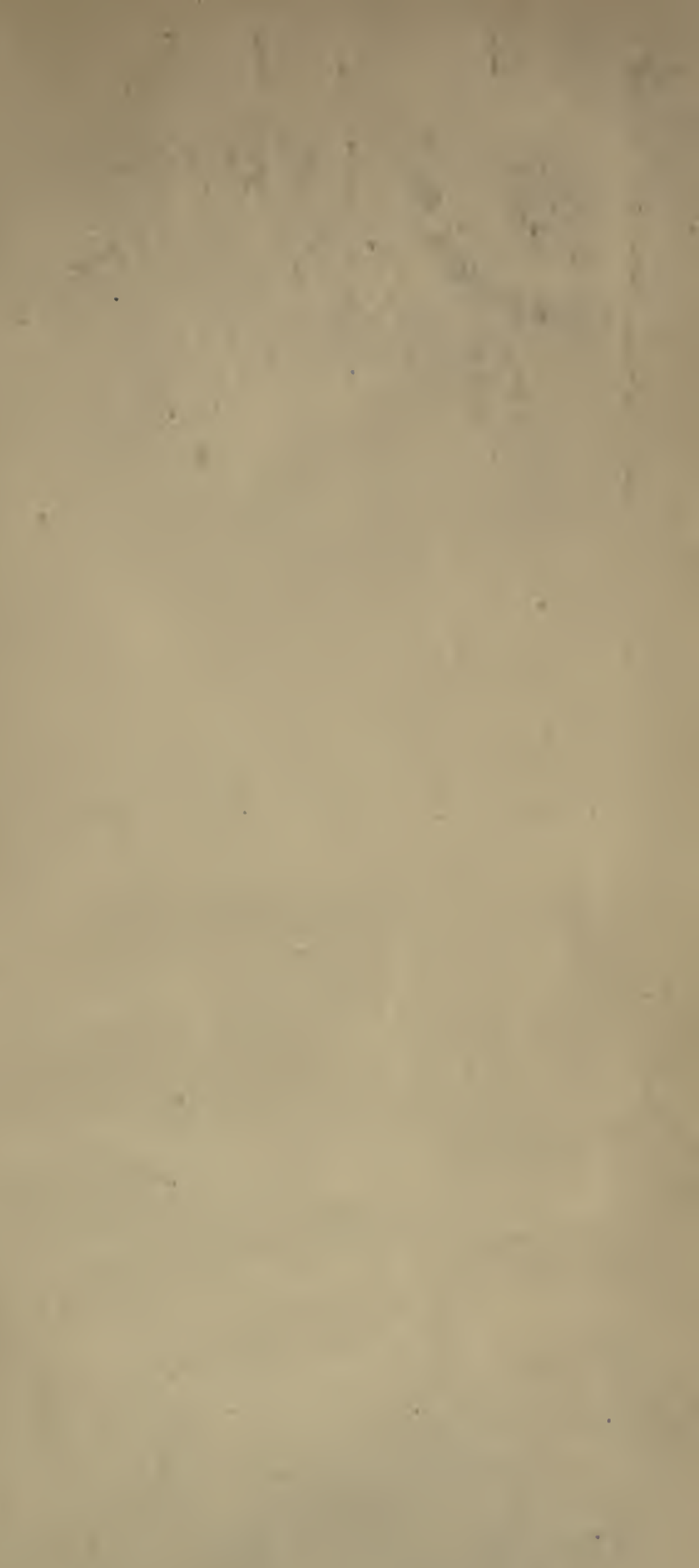


Digitized by the Internet Archive in 2007 with funding from Microsoft Corporation 
.

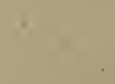

a

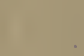

$y$

1

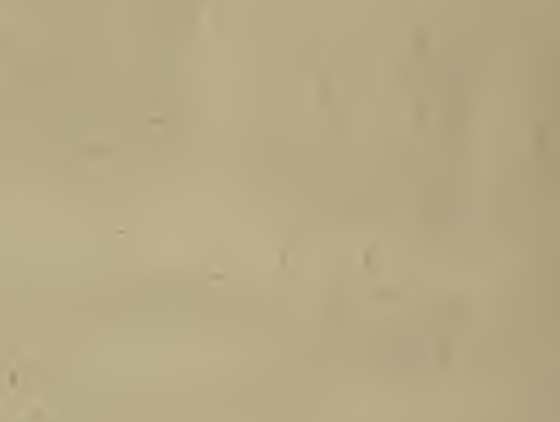

7

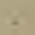




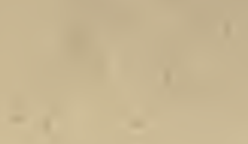




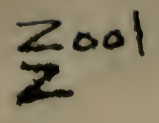

\section{REVISED LIST I}

OF THE:

\section{VERTEBRATED ANIMALS}

NOW OR LATELY LIVING

IN THE GARDENS

OF THE

\section{ZOOLOGICAL SOCIE'TY OF LONDON.}

\section{SUPPLEMENT,}

CONTAINING ADDITIONS RECEIVED IN 1872, 1873, AND 18T4.

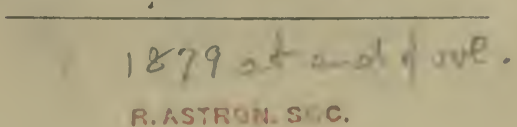

PRINTED FOR THE SOCIETY, ANI) SOLD AT THEIR HOUSE IN HANOVER SQUARE.

$$
\text { L O N D ON : }
$$

MESSRS. LONGMANS, GREEN, READER, AND JYEK PATERNOSTER ROW.

1875.

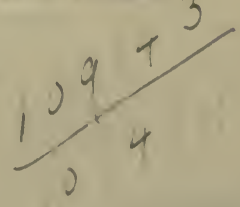




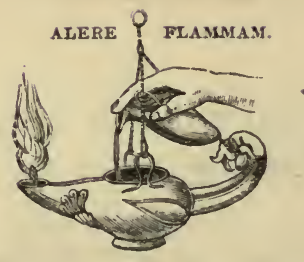

PRINTED BY TAYLOR AND FRANCIS, RED LION COURT, FLEET STREET. 


\section{PREFACE.}

Tuis Supplement contains the names of the animals reccived by the Zoological Society of London during the past three years which belong to species not included in the 'Revised List' published in 1872. It also gives the date of arrival and the mode of acquisition of each individual of such specics. The additional spccimens of spccics already inserted in the former list are not catalogued.

The subjoined is an approximatc account of the number of specics belonging to the five classes of Vertebrated Animals exhibited in the Society's Gardens since 1860-that is, during the past 14 years :-

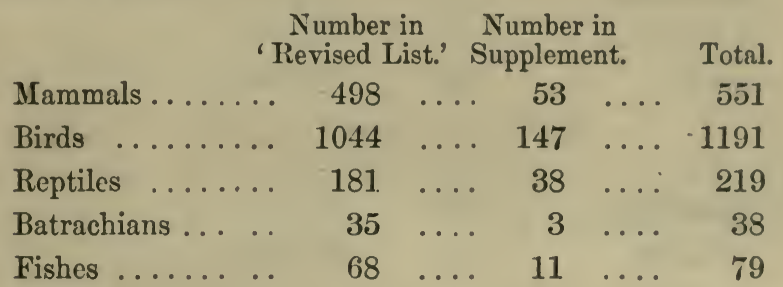

11 Hanover Square, W.

P. L. S.

July 28 th, 1875 . 


\section{CONTENTS.}

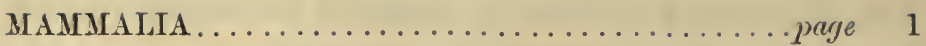

Quadrumana....page $1 \mid$ Rodentia ........... 6

Lemures ........ 3 Urigulata .......... 8

Chiroptera ...... 3 Edentata ......... 11

Insectivora ......4 4 Marsupialia........ 11

Carnivora........4 4

AVES.............................. 12

Passeres........ 12 | Columbs .......... 30

Cypseli ........20 20 Gallinæ ...........31

Pici ......... 20

Coccyges $\ldots \ldots 21$

Psittaci ........ 22

Accipitres ......2 26

Steganopodes..... 28

Herodiones .....2 28

Anseres ........29

Alectorides .......... 33

Grallæ ............. 34

Gaviæ ............. 35

Pygopodes ......... 36

Impennes.......... 36

Crspturi ........... 37

Struthiones......... 38

REPTILIA . . . . . . . . . . . . . . 39

Testudinata .... 39 Sauria ........... 42

Crocodilia .....4 42 Ophidia .........44

BATRACHIA .................... 47

PISCES ......................... 48 


\title{
LIST OF ANIMALS.
}

\author{
Class MAMMALIA.
}

Order QUADRUMANA.

\author{
Family CERCOPITHECIDÆ.
}

Genus Semnopithecus.

7 a. Semnopithecus obscurus, Martin. Dusky Monkey. Hab. Malacca.

a. Purchased, Jan. 26, 1874.

b. Purchased, Oct. 10, 1874.

Genus Colobus.

10a. Colobus bicolor (Wesmael). White-thighed Colobus. $H a b$. West Africa.

a. Purchased, March 28, 1873.

\section{Genus Cercopithecus.}

23 a. Cercopithecus campbelli, Waterh. Campbell's Monkey. $H a b$. West Coast of Africa.

a. Female. Presented by Mrs. E. Ives, June 15, 1872.

b. Purchased, Dec. 15,1874 . See P.Z.S. 1875 , p. 1. 
Genus Macacus.

39 a. Macacus rheso-similis, Sclater. Rhesus-like Monkcy. Hab. East Indies.

a. Nale. Purchased, Feb. 15, 1872. See P.Z.S. 1872, p. 495, pl. xxv., and P.Z.S. 1875 , p. 418.

46 a. Macacus rufescens, Anderson. Reddish Macaque. Hab. East Indies.

a. Male. Purchased, Feb. 14, 1872. See P.Z.S. 1872, p. 495, pl. $x \times r v$.

46 b. Macacus brunneus, Anderson. Brown Monkey. Hab. Burmah.

a. Male. Presented by Mr. Oscar Fraser, Feb. 14, 1872. Specimen figured, P.Z.S. 1872, p. 203, pl. xr.

b. Female. Presented by Lieut. Burne, Feb. 14, 1872. See P.Z.S. 1872 , p. 494.

c. Female. Purchased, Feb. 14, 1872. See P.Z.S. 1872, p. 494, and P.Z.S. 1874 , p. 652.

\section{Family CE1BIDAE.}

\section{Genus Mrcetes.}

68 u. Mycetes fuscus, Kuhl. Brown Howler. Hab. Brazil.

a. Male. Purchased, Nor. 8, 1872.

\section{Genus SaImaris.}

78 a. Saimarisœrstedi(Reinh.). Red-backed SquirrelMonkey. $H a b$. Central America.

a. Purchased, April 29, 1873. From Veragua. See P.Z.S. 1873, p. 434.

b. Female. Presented by W. F. Kelly, Esq., Sept. 5, 1874. From Guatemala. See P.Z.S. 1874, p. 495. 


\section{Family H HPALID A.}

\section{Genus Hapale.}

87 a. Hapale melanura (Geoffr.). Black-tailed Marmoset. Hab. South America.

a. Male. Purchased, Nov. 9, 1874. See P. Z. S. 1875, p. 419 , pl. L.

\section{Order LEMURES.}

Family LEMURID $Æ$.

Genus Lemur.

98 a. Lemur nigrifrons, Geoffr. Black-fronted Lemur. Hab. Madagascar.

a. Purchased, Aug. 5, 1874.

\section{Order CHIROP'TERA.}

Family PTEROPODIDA.

Genus Pteropus.

116 a. Pteropus formosus, Sclater. Formosan Fruit-Bat. Hab. Formosa.

$a, b$. Presented by the Rer. Mr. Ritchie, Jan. 9, 1873. See P.Z.S. 1873, p. 193, pl. xxir.

\section{Family VESPERTILIONID AE.}

\section{Genus Vespertilio.}

118 a. Vespertilio nattereri, Kuhl. Natterer's Bat. $H a b$. British Isles.

a, b. Presented by Lord Lilford, F.Z.S.. July 19, 1872. See P. Z. S. 1872, p. 790. 


\section{Family NOCTILIONID E.}

\section{Genus Taphozous.}

118 b. Tuphozous nudiventer (Rüpp.). Naked-bellied TombBat.

Hab. Africa.

a. Presented by Mr. W. Jamrach, Jan. 20, 1873. See P.Z.S. 1873 , p. 194.

\section{Order INSECTIVORA.}

Family ERINACEID A.

Genus Erinaceus.

120 a. Erinaceus collaris, Gray. Collared Hedgehog. Hab. N.W. India.

a-d. Presented by A. Anderson, Esq., F.Z.S., May 17, 1873. See P. Z. S. 1873 , p. 518.

\section{Order CARNIVORA.}

Family FELID $Æ$.

Genus Felis.

132 a. Felis servalina, Ogilby. Servaline Cat. $H a b$. West Africa.

a. Presented by Spencer Shield, Esq., Sept. 11, 1874. From Kinsembo, near Ambriz, Congo, West Coast of Africa. See P.Z.S. 1874, p. 495, pl. Lxm.

136 a. Felis planiceps, Vig. \& Horsf. Red Tiger Cat. Hab. Malacca.

a. Purchased, Dec. 14, 1872. See P.Z. S. 1873, p. 2.

136 b. Felis aurata, Temm. Rusty Tiger Cat.

Hab. Gold Coast, W. Africa.

a. Female. Presented by C. S. Salmon, Esq., Feb. 11, 1873.

See P. Z. S. 1873, p. 311. 


\section{Family VIVERRIDA:}

\section{Genus Viverricula.}

153 a. Viverricula schlegeli, Pollen. Schlegel's Civet. Hab. Comoro Islands.

a. Presented by C. E. Beusher, Esq., Oct. 28, 1872. From Johanna, Comoro Islands. See P.Z. S. 1872, p. 862.

\section{Family CANIDA.}

\section{Genus Canis.}

194a. Canis fulvicaudus, Lund. Red-tailed Fox. Hab. South America.

a. Purchased, Jan. 2, 1874 .

194 b. Canis fulvipes, Martin. Red-footed Fox. Hab. South America.

a. Presented by F. Grant, Esq., Oct. 9, 1874. From Pernambuco.

\section{Genus Nyctereutes.}

197 a. Nyctereutes procynides, Gray. Raccoon-like Dog. Hab. Amoorland.

a. Purchased, May 21, 1874. See P.Z.S. 1S74, p. 323, pl. L.

Family MUSTELID

\section{Genus Helictis.}

212 a. Helictis subaurantiaca, Swinhoe. Orange-bellied Helictis.

\section{Hab. China.}

a. Purchased, Nov, 26, 1874 . See P.Z.S. 1874, p. 666. 


\title{
Genus Meles.
}

214 a. Meles leptorhynchus, A. Milne-Edwards. Sharp-nosed Badger.

$H a b$. China.

a. Presented by R. Swinhoe, lisq., F.Z.S., Nov. 13, 1873. See P.Z. S. 1873, p. 762 .

\section{Order RODEN'TIA.}

\author{
Family SCIURIDÆ.
}

\section{Genus Sciurus.}

$242 a$. Sciurus griseopectus, Blyth. Grey-breasted Squirrel. Hab. China.

a. Male. Presented by R. Swinhoe, Esq., F.Z.S., Nov. 9, 1872. See P.Z. S. 1872 , F. 818.

244a. Sciurus tennenti, Layard. Tennent's Squirrel. Hab. Ceylon.

a. Male; b. Female. Presented by Hugh Nevill, Esq., July $9,1873$.

244. b. Sciurus macrurus, Erxl. Grizzled Hill-Squirrel. $H a b$. India.

a. Purchased, Dec. 4, 1873.

244 c. Sciurus macruroides, Hodgson. Black Hill-Squirrel. Hab. East Inảies.

a. Purchased, Nov. 11, 1873.

b. Purchased, Dec. 4, 1873.

247 a. Sciurus tristriatus, Waterh. Three-striped Squirrel. Hab. Ceylon.

a, b. Females. Presented by Capt. Forster, March 16, 1874. 
217 b. Sciurus erythropus, F. Cuv. White-banded Squirrel. Hab. West Africa. a, b. Presented by James R. Philpot, Esq., Nov. 21, 187\%.

Genus Tamias.

258 u. Tamias striatus (Limm.). Chipping Squirrel.

Hab. North America.

a, b. Purchased, Aug. 29, 1872.

\section{Genus Pteromys.}

258 b. Pteromys magnificus, Hodgs. Red-bellied Flying Squirrel.

Hab. Himalayas.

a. Purchased, April 11, 1872. See P.Z.S. 1872, p. 635, pl. L.

258 c. Pteromys leucogenys, Temm. White-cheeked Flying Squirrel.

Hab. Japan.

a. Female. Presented by Abel A. J. Gower, Esq., April 15, 1874. See P.Z.S. 1874, p. 247.

\section{Genus Sciuropterus.}

259 a. Sciuropterus fimbriatus, Gray. Grey Flying Squirrel. $H a b$. Northern India.

a. Presented by Capt. the Hon. G. Napier, Dec. 11, 1872.

\section{Genus Spermophilus.}

260 a. Spermophilus mongolicus, A. Milne-Edwards. Mongolian Souslik.

Hab. China.

a, b. Presented by R. Swinhoe, Es(1., F.Z.S., Nov. 13, 1873.

See P.Z. S. 1873, p. 762. 


\section{Family HYSTRICIDÆ.}

\section{Genus Dasyprocta.}

301 a. Dasyprocta antillensis, Sclater. West-Indian Agouti. Hab. St. Lucia, West Indies.

$a, b$. Presented by Neville Holland, Esq., Nov. 24, 1874. See P.Z.S. 1874, p. 666, pl. xxxxir.

301 b. Dasyprocta mexicana, De Saussure. Mexican Agouti. Hab. Mexico.

a. Presented by H. A. Marckmann de Lichtabbell, Esq., Feb. 19, 1874.

\section{Order UNGULA'IA.}

Suborder PERISSODACTYLA.

Family RHINOCEROTIDA.

Genus Rhinoceros.

315 a. Rhinoceros sondaicus, Cuv. Javan Rhinoceros. Hab. Java.

a. Male. Purchased, March 7, 1874. See P.Z.S. 1874, p. 182, pl. xxvIII.

315 b. Rhinoceros lasiotis, Sclater. Hairy-eared Rhinoceros. Hab. Chittagong.

a. Female. Purchased, Feb. 14, 1872. See P.Z.S. 1872. pp. 185, 493, pl. xxmI., et p. 790 .

315 c. Rhinoceros sumatrensis, Cuvier. Sumatran Rhinoceros. Hab. Malacca.

a. Female. Purchased, Aug. 21, 1872. See P.Z.S. 1872, p. 790, pl. LxviI. 


\section{Family BOVID Á.}

\section{Genus Gazella.}

366 a. Gazella muscatensis, Brooke. Muscat Gazelle.

Hab. Muscat.

a. Male. Presented by Major C. B. Evan Smith, Aug. 15, 1872. See P. Z.S. 1874, p. 141, pl. xxir.

b. Female. Deposited, Sept. 26, 1873.

c. Male. Presented by J. H. Bainbridge, Esq., May 26, 1874.

366 b. Gazella arabica, Hempr. et Ehr. Arabian Gazelle. Hab. Arabia.

a. Male; b. Female. Presented by Mrs. Benecke, July 25, 1874. From Aden. See P.Z.S. 1874, p. 494.

\section{Genus Oryx.}

397 a. Oryx beatrix, Gray. Beatrix Antelope.

Hab. Arabia.

a. Deposited, March 26, 1872. See P.Z. S. 1872, p. 603.

397 b. Oryx beisa (Rüpp.). Beisa Antelope. Hab. N.E. Africa.

a. Male. Presented by Admiral Arthur Cumming, May 28, 1874. See P.Z. S. 1874 , p. 323.

Family CERVIDÆ.

Genus Cervus.

435 a. Cervus virginianus, Gm. Virginian Deer. Hab. North America.

a. Male. Purchased, Aug. 27, 1861.

$b, c$. Male and Female. Receired in exchange, Feb. 19, 1873.

d. Female. Presented hy Capt. Bennett, May 17, 1873.

e. Male. Born in the Menagerie, May 21, 1873.

f. Male. Presented by N. M. Bateson, Esq., March 15, 1874.

\%. Male. Born in the Menagerie, June 27, 1874.

h. Female. Presented by Capt. E. N. Cobbett, Nov. 27, 1874. 
436 a. Cervus rufus, F. Cuv. Red Brocket.

Hab. Brazil.

a. Male; b. Female. Purchased, Mas 24, 1872. From Maranham.

c. Female. Received in exchange, June 29, 1874.

d. Female. Purchased, July 7, 1874.

438 a. Cervus campestris, F. Cuv. Pampas Deer.

Hab. South America.

a. Male; b. Female. Purchased, Dec. 23, 1874. From the Argentine Republic. See P.Z.S. 1875, p. 1.

\section{Genus Cervulus.}

439 a. Cervulus muntjac, Zimm. Indian Muntjac. Hab. India.

a. Male. Purchased, Nov. 6, 1874. From Saigon. Sec P.Z. S. 1874, p. 665.

440 a. Cervulus micrurus, Scl. Short-tailed Muntjac.

Hab. Ningpo, N. China.

a. Female. Purchased, Feb. 27, 1874. Sce P.Z.S. 1975, p. 421.

b. Male. Purchased, May 29, 1874 . See P.Z.S. 1875, p. 421, pl. LI. fig. 1 .

440 b. Cervulus sclateri, Swinhoe. Sclater's Muntjac. $H a b$. China.

$a, b$. Males. Purchased, May 29, 1874. See P. Z.S. 18 ‘t, p. 40 , pl. virr.

\section{Genus Hydropotes.}

441 a. Hydropotes inermis, Swinhoe. Chinese Water-Deer. Hab. China.

a. Male. Presented by R. Swinhoe, Esq., F.Z.S., May 8, 1873. See P.Z.S. 1873, p. 517.

b. Purchased, Jan. 1, 1874. See P.Z. S. 1874, p. 110.

c. Male. Deposited, Feb. 27, 1874. 


\section{Order EDEN'TA'TA.}

Family DASYPODIDA.

\section{Grenus Xenurus.}

4.53 a. Xenurus unicinctus (Linn.). Broad-banded Armadilo. Hab. Brazil.

a. Purchased, April 8, 1873. Sce P. Z. S. 1873, p. 434.

\section{Order MARSUPIALIA.}

\section{Family PHALANGISTIDA.}

\section{Genus Dromicia.}

470 a. Dromicia nana (Geoffr.). Dormouse Phalanger. Hab. Tasmania.

๘. Purchased, May 30, 1873.

b. Presented by W. F. Stratford, Esq., June 18, 1873.

\section{Family MACROPODIDAE.}

\section{Genus Dorcopsis.}

488 a. Dorcopsis luctuosa (D’Albertis). Mourning Kangaroo. $H a b$. South of New Guinea.

a. Female. Deposited, April 17, 1874. See P.Z. S. 1874, p. 247 , pl. xuII., and 1875 , p. 48. 


\section{Class AVES.}

\section{Order PASSERES.}

Family TURDID瓜.

Genus Turdus.

5 b. Turdus rufiventris, Vieill. Red-bellied Thrush. Hab. Buenos Ayres.

a. Purchased, Oct. 30, 1873.

Genus Srlvia.

$16 a$. Sylvia cinerea, Lath. Common Whitethroat. Hab. British Isles.

a. Purchased, May 3, 1872.

16 b. Sylvia atricapilla, Linn. Black-cap Warbler. Hab. Europe.

a. Presented by Lieut. J. L. Hearne, R.N., April 9, 1874.

Family PARIDE.

Genus Sitra.

33a. Sitta europea, Linn. White-bellied Nuthatch. $H a b$. N. Europe.

a. Purchased, Oct. 14, 1874.

\section{Genus Parus.}

$33 b$. Parus caruleus, Linn. Blue Tit.

Hab. British Isles.

$a, b$. Received in exchange, Jan. 4, 1872. 
33 c. P'urus cyanus, Pallas. Azure Titmouse.

Hab. N. Europe.

a. Purchased, Oct. 14, 1874.

33 d. Parus palustris, Linn. Marsh-Tit.

Hab. British Isles.

a. Nale; b. Female. Purchased, Jan. 31, 1872.

33 e. Parus major, Linn. Great Tit.

$H a b$. British Isles.

$a, b$. Receired in exchange, Jan. $4,1872$.

$33 f$. Parus ater, Linn. Cole Tit.

Hab. British Islands.

a-c. Purchased, May 5, 1873.

\section{Family CRATEROPODID A.}

\section{Genus Garrulax.}

39 b. Garrulax perspicillatus (Gm.). Masked Jay Thrush. $H a b$. China.

$a, b$. Purchased, July 22, 1874.

39 c. Garrulax picticollis, Swinhoe. Collared Jay Thrush. $H a b$. China.

a. Purchased, Oct. 30, 1873. See P. Z. S. 1873, p. 761.

\section{Genus Struthidea.}

$40 a$. Struthidea cinerea, Gould. Grey Struthidea. Hab. Australia.

$a, b$. Received in exchange, March 9, 1872. See P.Z.S. 1872, p. 603.

c. Purchased, Nor. 22, 1872 . 


\section{Family PYCNONOTIDA.}

\section{Genus Pycnonotus.}

43 a. Pycnonotus hamorrhous (Gm.). Red-vented Bulbul. Hab. India.

a. Purchased, May 9, 1872.

b. Purchased, April 25, 1873.

c. Deposited, June 26, 1874.

\section{Genus Phyllornis.}

44a. Phyllornis aurifrons, Temm. Malabar Grecn Bulbul. Hab. India.

a. Purchased, Jan. 12, 1874.

\section{Family NECTARINIID A.}

\section{Genus Zosterops.}

47 a. Zosterops lateralis (Lath.). Lateral White-cye. Hab. New Zealand. $a-f$. Presented by Mr. Bills, Ang. 31, 1872.

\section{Family TANAGRIDA.}

Genus Euphonia.

54a. Euphonia crassirostris, Sclater. Thick-billed Violet Tanager.

Hab. Central America.

a. Presented by Mr. Davidson, June 28, 1872.

\section{Genus Calliste.}

56 a. Calliste brasiliensis (Linn.). Blue-and-Black Tanager. Hab. Brazil. a-f. Purchased, Oct. 20, 1873. 
s(i b. Calliste melanonota (Vicill.). Black - slıouldered 'Tanager.

II $\iota b$. Brazil.

a. Purchased, Oct. 20, 1873.

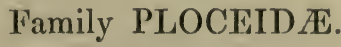

\section{Genus Pytelia.}

79 a. Pytelia phoenicoptera, Sw. Crimson-winged Waxbill. Hab. West Africa.

a. Purchased, Jan. 12, 1874.

b-d. Purchased, May 23, 1874.

\section{Genus Amadina.}

81 a. Amadina bicolor, Fraser. Cape-Palmas Finch. Hab. West Africa.

a, b. Purchased, April 3, 1872.

\section{Family FRINGILLID $A$.}

\section{Genus Paroaria.}

123 a. Paroaria capitata (Lafr. et D’Orb.). Yellow-billed Cardinal.

Hab. Chili.

a, b. Purchased, Nov. 11, 1873.

\section{Genus Passe R.}

139 a. Passer petronella (Bp.). Yellow-throated Sparrow. Hab. Africa.

a-cl. Purchased, Feb. 13, 1872.

139 b. Passer alario (Linn.). Alario Sparrow.

$H a b$. South Africa.

a, b. Purchased, Jan. 2, 1874. 


\section{Genus Srcalis.}

151 a. Sycalis luteola (Sparrm.). Yellowish Finch.

$H a b$. Brazil.

$a, b$. Purchased, July 11, 1873.

Genus Serinus.

$156 a$. Serinus canicollis (Sw.). Grey-necked Serin Finch. Hab. Cape of Good Hope.

a. Received in exchange, July 8, 1872.

\section{Genus Loxia.}

164a. Loxia pityopsittacus, Bechst. Parrot Crossbill. Hab. Europe.

a. Purchased, Dec. 9, 1873.

\section{Genus Eмвенiza.}

173 a. Emberiza chrysophrys (Pall.). Yellow-browed Buntıng. Hab. Japan.

a. Purchased, April 25, 1873. See P.Z. S. 1873, p. 434.

Genus Euspiza.

175 a. Euspiza rutila (Pall.). Red-backed Bunting. $H a b$. Japan.

a. Purchased, April 25, 1873. See P.Z. S. 1873, p. 434.

175 b. Euspiza americana, Gm. American Bunting. $H a b$. America.

a. Purchased, June 4, 1873.

175 c. Euspiza aureola (Pall.). Yellow-breasted Bunting. Hab. Japan.

a. Male. Received in exchange, Nov. 13, 1873. $b-e$. Purchased, Oct. 14, 1874. 


\section{Genus Melophus.}

175 d. Melophus melanicterus (Gm.). Crested Black Buntinğ. $H a b$. India.

a, b. Purchased, April 25, 1873.

Genus Zonotrichis.

176 a. Zonotrichia albicollis (Gm.). Whitc-throated SongSparrow.

Hab. North Amerieà.

$a, b$. Purchased, Feb. 16, 1872.

176 b. Zonotrichia pileata (Bodd.). Pileated Song-Sparrow. Hab. North Ameriea.

a. Received in exchange, July 8, 1872.

Genus Pipılo.

177 a. Pipilo erythrophthalmus (Linn.). Red-eyed GroundFinch.

Hab. North America.

a. Purchased, Feb. 16, 1872.

Family ALAUDID Æ.

Genus Alauda.

179 a. Alauda gulgula (Franklin). Indian Sky-Lark. Hab. India.

a. Purchased, June 18, 1872.

182 a. Alauda cristata (Linn.). Crested Lark. $H a b$. India.

a. Male; b. Female. Purchased, June 18, 1872. 


\section{Genus Mirapra.}

182 b. Mirafra affinis (Jerdon). Madras Bush-Lark. $H a b$. India.

a. Purchased, June 18, 1872.

\section{Genus Pyrrhulauda.}

184a. Pyrrhulauda verticalis (Smith). White-headed Bullfinch Lark.

$H a b$. South Africa.

a-c. Purchased, Sept. 26, 1873.

\section{Family ICTERIDA.}

\section{Genus Dolichonyx.}

189 a. Dolichonyx oryzivora (Linn.). American Rice-bird. Hab. North America. $a, b$. Purchased, June 14, 1873.

\section{Genus Xanthosomus.}

189 b. Xanthosomus flavus (Gm.). Yellow Trupial. Hab. Buenos Ayres. $a, b$. Purchased, Oct. 30, 1873.

\section{Genus Cassicus.}

192 a. Cassicus hamorrhous (Linn.). Red-rumped Hang-nest. $H a b$. South America.

a. Purchased, Sept. 13, 1873.

\section{Genus Icterus.}

195 a. Icterus vulgaris (Daud.). Common Hang-nest. Hab. South America.

a. Purchased, May 1, 1871.

$b-d$. Purchased, April 29, 1873.

e. Deposited, July 16, 1874. 
195 b. Icterus spurius (Limn.). Orehard Hang-nest.

Hab. North America.

a-d. P'urchased, July 25, 1873.

\section{Genus Aphobus.}

199 a. Aphobus chopi, Vieill. Chopi Starling.

Hab. Brazil.

a. Purchased, Jan. 11, 1872.

b. Purchased, May 14, 1872.

\section{Family STURNIDE.}

Genus Acridotineres.

209 a. Acridotheres mahrattensis (Sykes). Southern Brown Mynah.

$H a b$. Southern India.

a. Purchased, May 14, 1872. See Blyth, Ibis, 1872, p. 79.

b. Purchased, Sept. 26, 1873.

\section{Family CORVID瓜.}

\section{Genus Corvus.}

221 a. Corvus culminatus, Sykes. Large-billed Crow. Hab. India.

a. Presented by W. Dunn, Esq., May 28, 1873.

\section{Genus Cyanopolius.}

229 a. Cyanopolius cyaneus (Pall.). Blue Magpic. $H a b$. China.

a. Presented by the Rev. A. W. Petre, Oct. 28, 1873.

\section{Genus Cyanocorax.}

234a. Cyanocorax incas (Bodd.). Peruvian Blue Jay. Hab. Columbia. $a, b$. Purchased, June 17, 1872. 


\section{Genus Dendrocitta.}

239 a. Dendrocitta sinensis (Lath.). Chinese Tree-Pie. $H a b$. China.

a. Presented by R. Swinhoe, Esq., F.Z.S., Nov. 9, 1872. See P.Z. S. 1872 , p. 862.

Family DENDROCOLAPTID瓜.

Genus Furnarius.

255 a. Furnarius rufus (Gm.). Red Oven-bird. Hab. Buenos Ayres.

a. Purchased, Oct. 30, 1873.

\section{Order CYPSELI.}

\section{Family CAPRIMULGID瓜.}

\section{Genus Caprimulgus.}

256 a. Caprimulgus europaus, Linn. Common Nightjar. Hab. Europe.

$a, b$. Deposited, Aug. 9, 1872.

c. Presented by Mr. J. W. Slack, June 9, 1873.

\section{Order PICI.}

Family PICID瓜.

\section{Genus Picus.}

257 a. Picus minor, Linn. Lesser Spotted Woodpecker. Hab. British Isles.

a. Purchased, Sept. 2, 1872. 


\section{Family YUNGII) E.}

Genus Yunx.

261. Yunx torquilla, Linn. Wryneck.

Hab. Europe.

a. Purchased, July 16,1874 .

\section{Order COCCYGES.}

Family ALCEDINID AE.

Genus Halcyon.

269 a. Halcyon vagans (Less.). New-Zealand Kingfisher. Hab. New Zealand.

a-c. Received in exchange, Oct. 2, 1872.

Family BUCEROTID $\mathrm{E}$.

Genus Buceros.

271 a. Buceros lunatus, Temm. Lunated Hornbill. Hab. Java.

a. Purchased, Feb. 14, 1872.

276 a. Buceros malayanus, Raffles. Malayan Hornbill. Hab. Malacca.

a. Purchased, Feb. 17, 1874. Sec P.Z. S. 1874, p. 151.

Family CUCULIDÆ.

Genus Centropus.

293 a. Centropus phasianus (Lath.). Pheasant Coucal. Hab. New South Wales.

1.. Purchased, March 5, 1872. Sce P.Z. S. 1872, p. 603. 


\section{Family RAMPHASTIDA.}

Genus Ramphastos.

$296 a$. Ramphastos vitellinus, Licht. Sulphur-and-whitebreasted Toucan.

Hab. South America.

a. Purchased, March 7, 1872. See P.Z. S. 1872, p. 862.

\section{Genus Pteroglossus.}

300 a. Pteroglossus wiedi, Sturm. Maximilian's Aracari. Hab. Brazil.

a. Received in exchange, Nor. 22, 1872. See P.Z.S. 1872, p. 862 .

\section{Order PSI'TTACI.}

Family PSI'TTACID E.

Genus Cacatua.

315 a. Cacatua goffini, Finsch. Goffin's Cockatoo.

$H a b$. Queensland.

a. Purchased, July 31, 1862.

b. Presented by C. Turner, Esq., June 28, 1871.

c. Presented by F. J. Dean, Esq., F.L.S., Oct. 17, 1874. See P.Z. S. 1875 , p. 61, pl. x.

Genus ArA.

328 a. Ara hahni, Souancé. Hahn's Macaw. $H a b$. Guiana.

$a, b$. Purchased, Jan. 9, 1872. 


\section{Genus Conurus.}

333 a. Conurus uagleri (Gray). Wagler's Conure.

Hab. Venczuela.

a. Purchased, July 7, 1873.

351 a. Conurus cruentatus (Max.). Red-eared Conure.

Hab. South America.

a, b. Purchased, Sept. 26, 1872.

Genus Brotogerys.

356 a. Brotogerys tovi (Gm.). Tovi Parrakeet.

Hab. Columbia.

a. Purchased, June 28, 1872.

Genus Paleornis.

365 a. Palcornis erythrogenys (Blyth). Red-checked Parrakect.

$H a b$. Nicobar Islands.

a. Purchased, March 13, 1873.

Genus Euphema.

372 a. Euphema chrysogastra (Lath.). Orange-bellied GrassParrakeet.

Hab. South Australia.

$a-d$. Purchased, Feb. 11, 1873.

Genus Pyrriulopsis.

388 a. Pyrrhulopsis tabuensis (Lath.). Tabuan Parrakeet. Hab. Fiji Islands.

a. Purchased, July 7, 1873. See P.Z. S. 1873, p. 683. 


\section{Genus Cyanorhamphus.}

397 a. Cyanorhamphus alpinus (Buller). Alpine Parrakeet. Hab. New Zealand.

a, b. Purchased, Sept. 23, 1872. See P.Z. S. 1872, p. 795.

\section{Genus Pronus.}

415 a. Pionus sordidus (Linn.). Blue-throated Parrot. Hab. Venezuela. a, b. Purchased, Nov. 24, 1873.

\section{Genus Chrysotis.}

427 a. Chrysotis albifrons (Sparrm.). White-browed Amazon. Hab. Honduras.

a. Purchased, Jan. 27, 1873.

428 a. Chrysotis finschi, Sclater. Finsch's Amazon. Hab. W. Mexico.

a. Presented by Mrs. C. Chivers, March 9, 1874. See P. Z.S. 1874, p. 206, pl. xxxiv.

428 b. Chrysotis agilis (Linn.). Active Amazon. Hab. Jamaica.

a. Purchased, Nov. 24, 1873.

432 a. Chrysotis guildingi, Vigors. Guilding's Amazon. Hab. St. Vincent, West Indies.

a. Purchased, May 29, 1874. See P.Z. S. 1874, p. 324.

b. Purchased, Sept. 2, 1874 . See P. Z. S. 1875, p. 324 .

432 b. Chrysotis bouqueti, Bechst. Blue-faced Green Amazon. Hab. Honduras.

a. Purchased, May 6, 1874. See P.Z. S. 1874, p. 323, and 1875 , p. $61, \mathrm{pl}$. xI. 
436 a. Chrysotis amazonica (Linn.)*. Orange-winged Amazon. Hab. South America.

a. Purchased, April 29, 1873. From Cartagena.

\section{Genus Loriculus.}

416a. Loriculus asiaticus (Latham). C'eylonese Hanging Parrakeet.

Hab. Ceylon.

a, b. Purchased, Nov. 22, 1872.

Genus Lorius.

418 a. Lorius domicella (Linn.). Purple-capped Lory.

Hab. Moluccas.

a. Purchased, Jan. 24, 1872.

Genus Eos.

453 a. Eos tricolor (Steph.). Blue-tailed Lory.

Hab. Moluccas.

a, b. Purchased, Feb. 28, 1874.

453 b. Eos rubra (Gm.). Red Lory.

Hab. Moluccas.

a. Purchased, March 3, 1874.

453 c. Eos riciniata (Bechst.). Violet-necked Lory. Hab. Moluccas.

a. Purchased, Sept. 26, 1873. See P. Z. S. 1873, p. 684.

\section{Genus Chalcopsitta.}

453 d. Chalcopsitta scintillata (Temm.). Red-fronted Lory. Hab. New Guinea.

a. Purchased, Nov. 22, 1872. See P. Z. S. 1872 , p. 862.

* N.B. The species termed C. amazonica in the 'Revised List,' p. 200, should stand as C. astiva (Linn.). 


\section{Genus Trichoglossus.}

457 a. Trichoglossus cyanogrammus (Wagl.). Grcen-naped Lory.

Hab. Moluccas.

a. Purchased, Jan. 22, 1874.

457 b. Trichoglossus ornatus (Linn.). Ornamental Lory. Hab. Moluccas.

a. Purchased, May 16, 1873.

Genus Nestor.

461 a. Nestor notabilis, Gould. Keá, or Mountain-Parrot. Hab. New Zealand.

a. Presented by the Acclimatization Society of Canterbury, N. Z., Aug. 31, 1872. See P.Z.S. 1872, p. 795.

\section{Order ACCIPITRES.}

Family FALCONIDÆ.

Subfamily POLYBORINA.

Genus Milvago.

481 a. Milvago chimachima (Vieill.). Chimachima Milvago. Hab. Brazil.

a. Purchased, Jan. 20, 1874.

Subfamily AqUILINA.

Genus Buteo.

494 a. Buteo erythronotus (King). Red-backed Buzzard. Hab. South America.

a. Purchased, July 20, 1874. 


\section{Genus Unubitinga.}

494. 6. Urubilinga anthracina, Nitzsch. Ash-colourcd Buzzard. Hab. St. Vincent, West Indies.

a. Purchased, Nov. 26, 1874.

\section{Genus SPIZAËtus.}

$516 a$. Spizaëtus caligatus (Raffl.). Malayan Hawk Eagle. Hab. Burmali.

a. Presented by H. Fielden, Esq., Nov. 21, 1873.

\section{Genus Circä̈tus.}

519 a. Circaëtus gallicus (Gm.). Short-toed Eagle. Hab. Morocco.

(. Presented by Capt. P. Perry, March 18, 1873. From Saffi, Morocco.

\section{Genus Herpetotheres.}

519b. Herpetotheres cachinnans, Limn. Laughing Falcon. Hab. Brazil.

a. Purchased, June $30,1874$.

Subfamily FALCONIN

Genus Tinnunculus.

$543 a$. Tinnunculus sparverius (Linn.). American Kestrel. Hab. South America.

a. Purchased, Aug. 29, 1874.

\section{Subfamily ACCIPITRIN XE.}

Genus Accipiter.

549a. Accipiter melanoleucus, Smith. Black-and-white Sparrow-Hawk.

Hab. Africa.

a. Presented by Capt. Griffiths, Sept. 5, 1874. Captured at sea off Cape Verd. 


\section{Order STEGANOPODES.}

Family PLOTIDE.

Genus Plotus.

596 a. Plotus anhinga, Linn. American Darter.

Hab. Brazil.

a. Purchased, Dec. 28, 1872. From Maranham. See P.Z.S. 1873, p. 3.

\section{Order HERODIONES.}

Family CICONIIDA.

Genus Ciconia.

619 a. Ciconia boyciana, Swinhoe. Boyce's Stork.

Hab. Japan.

a, b. Presented by R. H. Boyce, Esq., Dec. 31, 1873. See P.Z.S. 1874 , p. 2 , pl. I.

Genus Leptoptilus.

626 a. Leptoptilus argala, Linn. Indian Adjutant. $H a b$. India.

$a, b$. Purchased, Jan. 26, 1874.

Family PLATALEID E.

Genus IвIs.

634a. Ibis nippon, Temm. Nippon Ibis. Hab. China.

a. Presented by R. Swinhoe, Esq., F.Z.S., Nor. 9, 1872. See P.Z. S. 1872 , p. 862 . 


\section{Order ANSERES.}

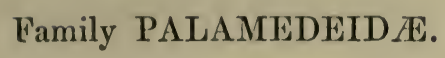

\section{Genus Chauna.}

645 a. Chauna chavaria (Linn.). Crested Screamer.

Hab. Buenos Ayres.

a. Presented by A. C. Maxwell, Esq., March 22, 1872. See P.Z. S. 1372 , p. 603.

b. Presented by Higford Burr, Esq., F.Z.S., March 25, 1872. See P.Z.S. 1872 , p. 603.

c. Presented by Mrs. Wilson, Feb. 28, 1873.

Family ANATIDAE.

Genus Rhodonessa.

706 a. Rhodonessa caryophyllacea (Latham). Pink-headed Duck.

Hab. India.

a. Male; b. Female. Purchased, Jan. 12, 1874. See P.Z.S. 1874, p. 110.

Genus Querquedula.

709 a. Querquedula falcata (Pallas). Falcated Teal. Hab. China.

a, b. Males. Purchased, Feb. 27, 1874. See P.Z. S. 1874, p. 152.

$c, d$. Males; e. Female. Deposited May 29, 1874.

$f$. Male; $g, h$. Females. Purchased May 29, 1874.

Genus Fuligula.

715 a. Fuligula mariloides (Vigors). Chinese Scaup Duck. Hab. China.

$a-c$. Females. From Ningpo. Presented by R.Swinhoe, Esq., F.Z.S., Feb. 22, 1873. See P.Z.S. 1873, p. 312. 
716 b. Fuligula rufina (Pallas). Red-crested Whistling Duck. $H a b$. India.

a. Male. Presented by E. Buck, Esq., June 3, 1874.

\section{Order COLUMB玉.}

Family CARPOPHAGID王.

Genus Carpophiga.

727 a. Carpophaga sylvatica, Tickell. Green Fruit-Pigeon. $H a b$. India.

$a, b$. Received in exchange, Sept. 9, 1874 .

Family COLUMBID 2.

Genus Ianthenas.

$756 a$. Ianthæenas leucolama, Gray. White-throated Violct Pigeon.

Hab. Moluccas.

a. Purchased, March 6, 1874. See P. Z.S. 1875, p. 367.

Genus Macropygia.

766 a. Macropygia phasianella (Temm.). Pheasant-tailed Pigeon.

Hab. New South Wales.

$a, b$. Purchased, Jan. 26, 1874.

Genus Turtur.

767 a. Turtur suratensis, Gm. Spotted Turtledove. $H a b$. India. $a, b$. Received in exchange, March 5, 1874. 


\section{Genus Peristera.}

777 a. Peristera geoffroii ('Temm.). Geoffroy's Dove. Hab. Brazil.

a, b. Purchased, Oct. 19, 1874. From the Island of Fernando de Noronha.

\section{Genus Zenaida.}

778 a. Zenaida martinicana (Bр.). Martinican Dove.

Hab. West Indies.

a-f. Presented by J. H. Hawtayne, Esq., July 9, 1873. From Union Island, one of the Grenadines.

テr8 $b$. Zenaida leucoptera (Linn.). White-winged Zenaida Dove.

Hab. West Indies.

$a, b$. Receired in exchange, June 15, 1874.

\section{Order GALLIN $\nRightarrow$.}

Family TETRAONIDE.

\section{Genus Tetrao.}

811 a. Tetrao phasianellus, Linn. Sharp-tailed Grouse.

Hab. North America.

a. Received in exchange, May 9, 1874.

\section{Family PHASIANIDÆ.}

Genus Coturnix.

836 a. Coturnix chinensis (Linn.). Chinese Quail. $H a b$. China.

a, b. Purchased, April 25, 1873.

Genus Odontophorus.

812 a. Odontophorus dentatus (Temm.). Capocira Partridge. Hab. Brazil.

a. Purchased, March 20, 1873. 


\section{Genus Callipepla.}

847 a. Callipepla gambelli (Nutt.). Gambel's Partridge. $H a b$. California.

$a, b$. Received in exchange, Aug. 29, 1872. See P.Z.S. 1872, p. 795 .

\section{Genus Caccabis.}

850 a. Caccabis petrosa, Gm. Barbary Partridge. Hab. North Africa.

a. Male; $b$, $c$. Females. Presented by Saville G. Reed, Esq., F.L.S., June 7, 1874. From Spain.

\section{Genus Pucrasia.}

868 a. Pucrasia darwini, Swinh. Darwin's Pucras.

Hab. China.

a, b. Males. Deposited, Jan. 1, 1874.

c. Male. Deposited, April 1, 1874.

\section{Genus Gallus.}

882 a. Gallus stanleyi (Gray). Ceylonese Jungle-fowl.

Hab. Ceylon.

a. Male; b. Female. Presented by H. Bayley, Esq., Sept. 10, 1873. See P. Z. S. 1873, p. 683.

$c, d$. Hatched in the Gardens, May 19, 1874 .

$e$. Hatched in the Gardens, June 5, 1874.

$f-h$. Hatched in the Gardens, July 3, 1874.

\section{Family CRACIDÆ.}

Genus Pipile.

919 a. Pipile cujubi (Pelz.). Amazonian Guan.

$H a b$. Lower Amazons.

a-c. Purchased, May 25, 1873. See P. Z. S. 1873, p. 518.

\section{Genus Ortalida.}

$921 a$. Ortalida albiventris (Wagl.). White-bellied Guan.

Hab. Brazil.

a. Purchased, Dec. 18, 1872. 
921 b. Ortalida vetula (Wagl.). Mexican Guan.

Hab. Mexico.

a. Purchased, May 15, 1873.

921 c. Ortalida garrula (Humb.). Chattcring Guan.

Hab. U. S. of Columbia.

a. Presented by George Dawson Rowley, Esq., F.L.S., Aug. 26, 1874. From Cartagena.

\section{Fanily TURNICID $\approx$.}

\section{Genus Turnix.}

928a. Turnix lepurana, Smith. Lepurana Hemipode.

$H a b$. West Africa.

$a, b, c$. Purchased, Feb. 19, 1873.

d. Purchased, Sept. 26, 1873.

\section{Order ALEC'TORIDES.}

Family OTID在.

Genus Eupodotis.

934a. Eupodotis denhami (Children). Denham's Bustard. $H a b$. Wesern Africa.

a. Presented by C. D. O'Connor, Esq., March 19, 1872. See P.Z. S. 1872, p. 603.

b. Presented by H.E. Governor Ussher, March 19, 1872. See P. Z.S. 1872 , p. 603.

\section{Family PSOPHIID瓜.}

\section{Grenus Psophia.}

940a. Psophia leucoptera, Spix. White-backed Trumpeter. Hab. Amazons. a. Deposited, Oct. 6, 187 t. 


\section{Family GRUIDÆ.}

Genus Grus.

947 a. Grus leucauchen, Temm. White-necked Crane. Hab. Japan. a, b. Purchased, April 17, 1873. See P.Z. S. 1873, p. 434.

Family RALLID 2 .

\section{Genus Porphyrio.}

970 a. Porphyrio indicus (Horsf.). Indian Porphyrio. Hab. Eastern Asia.

a. Presented by the Rev. J. S. Whitmee, C.M.Z.S., May 28, 1873. From the Navigators' Islands. See P.Z.S. 1873 , p. 518.

\section{Order GRALLA.}

\section{Family CHARADRIIDA.}

Genus Egialitis.

992 a. Agialitis hiaticula (Linn.). Ringed Plover.

Hab. British Islands.

a. Purchased, July 14, 1873.

\section{Family SCOLOPACID出。}

\section{Genus Numenius.}

1000 a. Numenius femoralis, Peale. Pacific Curlew. Hab. Pacific Islands.

$a, b$. Presented by the Rev. J. S. Whitmee, C.M.Z.S., May 27, 1874. From Quiros Island, S. Pacific. See P.Z.S. 1874 , p. 323. 


\section{Genus Himantopus.}

1007 a. Himantopus nigricollis, Vicill. Black-necked Stilt. Hab. S. America.

a. Purchased, Dec. 28, 1872. From Maranham. See P.Z.S. 1873, p. 2 .

\section{Genus Recurvirostra.}

1007 b. Recurvirostra avocetta, Linn. European Avocet.

$H a b$. Europe.

$a-d$. Purchased, Aug. 21, 1874.

\section{Order GAVIæ.}

Family LARID E.

Genus Lestris.

1008a. Lestris pomatorhinus, Temm. Pomatorhine Skua. Hab. Europe.

1. Purchased, Nor. 20, 1874.

\section{Genus Lakus.}

1010a. Larus leucopterus (Faber). Iceland Gull. $H a b$. Northern Europe.

a. Presented by B. L. Smith, Esq., Oct. 7, 1873.

1014a. Larus canus (Linn.). Common Gull.

$H a b$. British Isles.

a. Presented by Dr. Stewart, Aug. 16, 1872.

b. Presented by Mr. W. K. Stanley, Feb. 18, 18 74 .

c. Presented by M. F. W. Martin, Esq., May, 28, 1874.

1014b. Larus audouini, Payr. Audouin's Gull.

$H a b$. Sardinia.

$a, b$. Presented by Lord Lilford, F.L.S., June 29, 1874. See P.Z.S. 1874, p. 494. 


\section{Genus Sterna.}

1019a. Sterna cantiaca (Gm.). Sandwich Tern.

Hab. Europe.

a-d. Purchased, Aug. 21, 1874.

\section{Family PROCELLARIID E.}

Genus Purfinus.

1022 a. Puffinus anglorum (Boic). Manx Shearwater.

Hab. British Isles.

a. Presented by Dr. Bree, F.Z.S., Sept. 10, 1873.

b. Deposited, Sept. 19, 1873.

\section{Genus Daption.}

1022b. Daption capensis (Linn.). Cape-Petrel. Hab. South Atlantic.

a. Purchased, Oct. 15, 1873. From Manilla.

\section{Order PYGOPODES.}

Family ALCID E.

\section{Genus Uria.}

1027 a. Uria troile, Linn. Common Guillemot.

Hab. British Isles.

$a, b$. Presented by W. H. Scratten, Esq., F.Z.S., Sept. 25, 1872.

c-e. Purchased, Aug. 21, 1873.

$f-k$. Presented by Sir H. Dalrymple, Bart., July 22, 1874.

\section{Order IMPENNES.}

Family SPHENISCID $\mathbb{E}$.

Genus Pygosceles.

1028 a. Pygosceles taniatus (Peale). Gentoo Penguin. $H a b$. Falkland Islands.

d. Purchased, Gct. 22, 1874. See P. Z. S. 1874, p. 605. 
Genus Eunypes.

1030 a. Eudyptes chrysocome (Forst.). Rock-hopper Penguin. Hab. Falkland Islands.

a. Presented by J. M. Dean, Esq., July 1, 1873. See P.Z. S. 1873 , p. 683.

\section{Order CRYP'IURI.}

Family TINAMID E.

Genus Tinamus.

1030 b. Tinamus solitarius (Vieill.). Solitary 'Tinamou. Hab. Brazil.

a, b. Received in exchange, Oct. 13, 1873.

c. Purchased, Oct. 15, 1874.

c. Received in exchange, Dec. 2, 1874.

Genus Crypturus.

103: a. Crypturus noctivagus, Max. Banded Tinamou. Hab. Brazil. (l-e. Purchased, Aug. 29, 1872. f-h. Purchased, Oct. 21, 1873.

103: b. Crypturus obsoletus (Temm.). Obsolcte Tinamou. Hab. Brazil.

a, b. Received in exchange, Oct. 21, 1873.

\section{Genus Nothura.}

103: c. Nothura maculosa, Temm. Spotted Tinamou. Hab. Bucnos Ayres.

$a, b$. lieceired in exchange, Aug. 8, 1873.

I, e. Purchased, Oet. 21, 1873. 
Genus Rhynchotus.

1033 b. Rhynchotus perdicarius (Kittl.). Chilian Tinamou. Hab. Chili.

a. Purchased, Jan. 10, 1873.

b, c. Purchased, Oct. 21, 1873.

\section{Order STRUTHIONES.}

Family CASUARIIDE.

Genus Casuarius.

1039 a. Casuarius uniappendiculatus, Blyth. One-wattled Cassowary.

$H a b$. New Guinea.

a. Presented by Capt. Moresby, of H.M.S. ' Basilisk,' Aug. 25, 1874. See P.Z.S. 1874, p. 495, et 1875, pl. xx.

1040 a. Casuarius westermanni, Scl. Westerman's Cassowary.

Hab. New Guinea.

a. Received in exchange, Sept. 20,1871. Specimen figured as Casuarins kaupi, P.Z. S. 1872, p. 147, pl. Ix. See P.Z. S. 1875 , pl. xix.

1040 b. Casuarius picticollis, Scl. Painted-necked Cassowary. $H a b$. South of New Guinea.

a. Purchased, May 27, 1874. See P. Z. S. 1875, pl. xvirr.

\section{Family APTERYGID E.}

Genus Apteryx.

1042a. Apteryx australis, Shaw*. Kiwi.

$H a b$. South Island, New Zealand.

a. Receiped in exchange, Oct. 10, 1872. See P.Z, S. 1872, p. 861 .

* The species thus termed in the "Revised List" (p. 323) should stand as $A$. mantelli, Bartlett. 


\section{Class REP'TILIA.}

\section{Order 'TES'I'UDINA'I'A.}

Family TESTUDINIDA.

\section{Genus Testudo.}

3a. Testudo stellata, Schw. Starred Tortoise. $H a b$. India.

a, b. Presented by W. Vincent Legge, Esq., R.A., C.M.Z.S., Nov. 2, 1872. From Ceylon.

c. Presented by Capt. Willkinson, Dec. 11, 1872.

d, e. Purchased, June 16, 1872.

$f, g$. Presented by Capt. C. S. Sturt, C.M.Z.S., June 10, 1873.

h. Presented by Capt. C. S. Sturt, C.M.Z.S., July 29, 1873.

$i-l$. Presented by H. P. Le Mesurier, Esq., Sept. 23, 1873.

m. Presented by Capt. Smelley, Mar. 9, 1874.

3 b. Testudo mauritanica, D. \& B. Algerian 'Tortoise.

$H a b$. Southern Europe and North Africa.

a. Deposited, June 28, 1872.

b. Presented by W. Heslop, Esq., Sept. 17, 1872. From the Crimea.

c, c. Presented by E. Cavendish Taylor, Esq., F.Z.S., March 6, 1873. From Algeria.

$e, f$. Presented by Capt. Perry, March 18, 1873.

$y, h$. Presented by the Hon. E. Ellis, Nov. 29, 1873.

i-n. Presented by Sir John Drummond Hay, K.C.B., April 4, 1873. From Barbary.

4a. Testudo semiserrata, Smith. Semiserrated Tortoise. Hab. S. Africa.

(1-c. Presented by Dr. George Grey, C.M.Z.S., Jan. 25, 1872. See P. Z. S. 1872 , p. 184. 
4.b. Testudo elongata, Blyth. Burmese Tortoise. Hab. Burmah. $a-d$. Presented by Dr. John Anderson, F.Z.S., May 16, 1873.

\section{Genus Homopus.}

10 a. Homopus areolatus (Thunb.). Areolated Tortoise.

Hab. Cape colony.

a-d. Presented by Dr. George Grey, C.M.Z.S., Jan. 25, 1872.

From Cradock. See P.Z.S.1872, p. 184.

\section{Family EMYDIDÆ.}

Genus Exys.

15 a. Emys europaa (Schneider). European Terrapin.

$H a b$. Italy.

$a-d$. Purchased, April 26, 1873.

$e-h$. Received in exchange, May 2, 1873.

i-o. Deposited, July 27, 1873.

$p$-s. Deposited, May 9, 1874.

t. Deposited, Sept. 11, 1874.

\section{Genus Cuora.}

15 b. Cuora amboinensis (Daud.). Amboina Box Tortoise. Hab. India.

a. Presented by Dr. John Anderson, F.Z.S., May 16, 1873.

\section{Genus Clemmys.}

17 a. Clemmys floridana (Le Conte). Floridan Terrapin. $H a b$. North America.

a. Presented by the Smithsonian Institution, Washington, U.S. A., June 4, 1874. From St. John's River.

19a. Clemmys serrata (Daud.). Serrated Terrapin. Hab. North America.

$a-d$. Presented by the Smithsonian Institution, Washington, U.S. A., June 11, 1874. From North Carolina. See P.Z.S. 1874 , p. 494 . 
20 a. Clemmys decussata (Bell). Hicotec Terrapin.

Hab. Jamaica.

a, b. Purchased, Jan. 28, 1872.

20 b. Clemmys punctularia (Daud.). Rough Terrapin.

$H a b$. Trinidad.

a-c. Presented by F. Chittenden, Esq., C.M.Z.S., Oct. 26, 1873.

29 a. Clemmys trijuga, Schweig. Ceylonese Terrapin.

$H a b$. India.

a, b. Presented by Capt. Wilkinson, Dec. 11, 1872. From Ceylon.

296. Clemmys unicolor, Gray. Black-headed Terrapin.

Hab. China.

a-c. Presented by R. Swinhoe, Esq., F.Z.S., May 8, 1873. See P.Z.S. 1873, p. 517, pl. xurv.

29 c. Clemmys reevesi (Gray). Reeve's Terrapin.

Hab. China.

a-c. Presented by R. Swinhoe, Esq., F.Z.S., May 8, 1873. See P.Z.S. 1873 , p. 517.

\section{Family TRIONYCHID}

\section{Genus Trionyx.}

51 a. Trionyx ferox, Schneid. North-American Trionyx. Hab. North America.

a. Presented by the Smithsonian Institution, Washington, U.S. A., June 4, 1874. From Lake Michigan. See P.Z. S. 1874 , p. 494. 


\section{Order CROCODILIA.}

\section{Family CROCODILIDA.}

\section{Genus Jacare.}

57 a. Jacare latirostris (Daud.). Broad-snouted Caiman. Hab. South America.

a. Purchased, April 29, 1873. From Baranquilla.

b. Presented by Capt. Turner, Sept. 15, 1874 .

\section{Genus Crocodilus.}

59a. Crocodilus frontatus, Murray. Broad-fronted Crocodile. $H a b$. West Africa.

a. Presented by J. J. Monteiro, Esq., C.M.Z.S., Junc 26, 1873. From the river Bonny.

b. Purchased, July $17,1873$.

\section{Genus Gavialis.}

63a. Gavialis gangeticus (Gm.). Gavial. $H a b$. Ganges.

a. Presented by Dr. John Anderson, Dec. 11, 1872. Sce P.Z.S. 1873 , p. 1.

b. Deposited, Dec. 20, 1873.

\section{Order SAURIA.}

Family AGAMID E.

Genus Agama.

70 a. Agama colonorum (Daud.). Spinose Lizard.

Hab. North-west Africa.

a. Purchased, June 7, 1873.

70 b. Agama occipitalis, Gray. Occipital Lizard.

Hab. W. Africa.

a. Presented by Lady Cust, June 29, 1873. 
Genus Uromastix.

7la. Uromastix microlepis, Blanford. Small-scaled Mastigure.

Hab. Busreh, Persian Gulf.

a-c. Presented by Capt. Phillips, Aug. 4, 1874. See P.Z.S. 1874, pp. 495, 658, pl. Lxxx.

71 b. Uromastix hardwickii, Gray. Hardwicke's Mastigure. $H a b$. India.

a, b. Presented by Lieut.-Col. C. S. Sturt, C.M.Z.S., Dec. 23, 1874.

Genus LyriocephaLus.

71c. Lyriocephalus scutatus (Linn.). Knob-nosed Lizard. Hab. Ceylon.

$a-e$. Presented by H. M. Moseley, Esq., March 11, 1872. See P.Z. S. 1872, p. 603.

\section{Genus Am pнibolurus.}

72a. Amphibolurus angulifer (Gray). Lozenge-marked Lizard. Hab. New Holland.

a. Purchased, June 2, 1873.

b. Purchased, July $31,1874$.

\section{Family LACERTIDA.}

Genus Lacerta.

92 a. Lacerta muralis, Merr. Wall-Lizard. Hab. Europe.

a, b. Presented by F. Coleman, Esq., May 9, 1872.

93 a. Lacerta lilfordi, Günther. Lilford's Lizard. Hab. Minorca.

$a, b$. Presented by Lord Lilford, F.Z.S., June 29, 1874. See P. Z. S. 1874, p. 494 . 


\section{Genus Psammodromus.}

94a. Psammodromus hispanicus, Fitz. Spanish Sand-Lizard. Hab. Spain.

$a, b$. Presented by E. Cavendish Taylor, Esq., F.Z.S., April 16, 1873. From Tangiers.

Family SCINCID $\approx$.

Genus Macroscincus.

97 a. Macroscincus cocteauii (Dum. et Bibr.). Cocteau's Skink.

$H a b$. Cape-Verd Islands.

a, b. Presented by Prof. Barboza du Bocage, F.M.Z.S., Oct. 14, 1874. See P.Z.S. 1873 , p. 703 , et 1874 , p. 495.

Genus Hrnulia.

102 a. Hinulia whitei, Gray. White's Skink.

$H a b$. Australia.

a-c. Purchased, July 31, 1874.

\section{Family SEPID $\mathbb{E}$.}

Genus SEPS.

106 a. Seps tridactylus, Laur. Three-toed Seps.

$H a b$. S. Europe.

a-c. Presented by W. C. Tait, Esy., July 30, 1872. From Portugal.

\section{Order OPHIDIA. \\ Family COLUBRID瓜.}

Genus Coronella.

125 a. Coronella phocarum, Günth. Robben-Island Snake.

$H a b$. Robben Island, South Africa.

a. Presented by the Rev. G. H. R. Fisk, Sept. 24, 1872. See Giinther, P. Z.S. 1872 , p. 795 et p. 836. 
b, c. Presented by the Rev. (G. II. R. Fisk, Aug. 29, 1873. See P.Z.S. 1873, p. 68:3.

d-f. Presented by the Rev. G. H. R. Fisk, Nov. 4, 1873.

\section{Genus Liopirs.}

129 a. Liophis merremii (Max.). Merrem's Snake. Hab. Monte Video.

a. Purchased, Jan. 17, 1874.

\section{Genus Zamenis.}

132 a. Zamenis caudilineatus, Günth. Lineated Snake. Hab. Asia Minor.

a. Purchased, June 10, 1872.

\section{Genus Coryphodon.}

133 a. Coryphodon testaceus (Say). Testaceous Snake. $H a b$. California.

a, b. Presented by A. Forrer, Esq., Aug. 20, 1873.

\section{Genus Tropidonotus.}

138 a. Tropidonotus leberis (Linn.). Seven-banded Snake. Hab. North America.

$a, b$. Purchased, Aug. 6, 1872.

$c-i$. Bred in the Gardens, Aug. 27, 1872.

\section{Genus Pituophis.}

141 a. Pituophis catenifer (Blainv.). Chained Snake. Hab. California.

๙. Presented hy A. Forrer, Esq., Aug. 20, 1873. 


\section{Family PSAMMOPHIIDE.}

\section{Genus Psammophis.}

150 a. Psammophis elegans, Shaw. Slender Sand-Snake. $H a b$. West Coast of Africa. $a-e$. Presented by C. B. Mosse, Esq., C.M.Z.S., July 4, 1872.

d. Purchased, June 21, 1873.

\section{Family CROTALID $\approx$.}

\section{Genus Crotalus.}

177 a. Crotalus miliarius (Linn.). Smaller Rattlesnake. Hab. Canada.

a. Presented by J. W. James, Esq., Nov. 30, 1873. 


\section{Class BATRACHIA.}

\section{Order ANURA.}

\section{Family BUFONIDA.}

Genus Buro.

5 a. Bufo agua, Latr. Giant Toad.

Hab. Brazil.

a. Presented by Mr. D'Arcy, Nov. 19, 1874.

\section{Family BOMBINATORIDÆ.}

Genus Bombinator.

12 a. Bombinator pachypus, Bp. Italian Fire-bellied Toad. $H a b$. Italy.

$a, b$. Presented by Prof. H. Giglioli, C.M.Z.S., May 22, 1872.

\section{Family HYLID $A$.}

Genus Hyla.

15 a. Hyla versicolor (Leconte). Changeable Tree-Frog. Hab. North America.

a. Presented by Prof. Rolleston, Nov. 10, 1873. 


\section{Class PISCES.}

Family SQUALID $\approx$.

Genus Scyllium.

2a. Scyllium stellare (Linn.). Large Spotted Dogfish. Hab. British Seas.

a, b. Purchased, July 28, 1873.

Genus Acanthias.

2b. Acanthias vulgaris, Risso. Picked Dogfish.

Hab. British Seas.

a-c. Purchased, July 18, 1872.

$d-i$. Received in exchange, Jan. 7, 1873.

Family TRACHINID E.

Genus Trachinus.

13 a. Trachinus vipera, C. \& V. Lesser Weever.

$H a b$. British Isles.

- $a-c$. Purchased, April 12, 1872.

Family GADID $\approx$.

Genus Gadus.

32 a. Gadus merlangus, Linn. Whiting.

Hab. British Seas. 
3:2 ४. Gaclus pollachins, Linn. 'The Pollack.

Hab. British Seas.

\section{Family PLEURONICTID As.}

Genus Rномвus.

35 a. Rhombus lavis (Limn.). Brill.

Hab. British Seas.

Family CYPRINIDA.

Genus Barbus.

51 a.. Burbus vulgaris, Fleming. Barbel.

- Hab. British fresh waters.

Genus Leuciscus.

55 a. Leuciscus cephalus (Linn.). Chub. Hab. British fresh waters.

Genus Aвкамrs.

5̌6a. Abramis brama, Linn. Bream.

Hab. British fresh waters.

Family PETROMYZONTID A.

Genus Petromyzon.

68 u. Petromyzon marinus, Linn. Sea-Lamprey. Hab. British Seas.

a. Purchased, April 12, 1872. 


\section{Family BRANCHIOSTOMATIDA.}

\section{Genus Branchiostoma.}

69. Branchiostoma lanceolatum (Pallas). Lancelet. $H a b$. Coast of Italy.

$a-r$. Presented by the Director of the Zoological Station, Naples. 


\section{N I) E X.}

Abramis brama $\quad$ Page 49 Acanthias vulgaris ... 48 Accipiter melanoleucus 27 Acridotheres mahrat-

tensis. 19

Adjutant, Indian..... 28 Egialitis hiaticula ... 34 Agama colonorum ... 42 - occipitalis ..... 42 Agouti, Mexican ...... 8 West-Indian ... 8 Alauda cristata ...... 17 - gulgula ......... 17

Amadina bicolor ...... 5

Amazon, Active ...... 24

_-, Blue-faced green 24 , Finsch's ......... 24

—, Guilding's ...... 24

- Orange-winged 25

- White-browed . 24

Amphibolurus angulifer.................... 43

Antelope, Beatrix ... 9

- Beisa ............ 9

Aphobus chopi ......... 19

Apteryx australis...... 38

Ara hahni.............. 22

Aracari, Maximilian's 22

Armadillo,Broad-ban-

ded

Avocet ................. 35

Badger, Sharp-nosed 5

Barbel ................. 49

Barbus vulgaris ...... 49

Bat, Naked-bellied Tomb-............ 4

- Natterer's ...... 3

Bombinator pachypus 47

Branchiostoma tanceolatum ............ 50

Bream ............... 49

Brill .................... 49

Brocket, Red ........ 10
Brotogerys tovi........ 23

Bufo agua............. 47

Bulbul,MalabarGreen 14 Red-vented ... 14

Bunting, American... 16

—, Crested black... 17

$\longrightarrow$, Red-backed ... 16

—, Yellow-breasted 16

- Yellow-browed. 16

Buceros lunatus ..... 21

- malayanus ...... 21

Bustard, Denham's ... 33

Buteo erythronotus ... 26

Buzzard, Ash-colour-

ed ................... 27

$\longrightarrow$, Red-backed ... 26

Cacatua goffini......... 22

Caccabis petrosa ...... 32

Caiman, broad-snout-

ed .................... 42

Callipepla gambelli... 31

Calliste brasiliensis ... 14

- melanonota ...... 15

Canis fulvicaudus ... 5

- fulvipes ......... 5

Caprimulgus europe-

us .................... 20

Cardinal, Yellow-bill-

ed .................... 15

Carpophaga sylvatica 30

Cassicus homorrhous . 18

Cassowary, One-wat-

tled ................. 38

$\longrightarrow$, Painted-necked 38

—, Westerman's ... 38

Casuarius picticollis.. 38

- uniappendicula.

tus.................... 38

- westermanni ... 38

Cat, Servaline ........ 4

$\longrightarrow$, Red Tiger ...... 4

—, Rusty Tiger ... 4

Centropus phasianus . 21
Page

Cercopithecus camp-

belli ................... 1

Cervulus micrurus ... 10

- muntjac ......... 10

- sclateri .......... 10

Cervus campestris ... 10

Crufus ............ 10

virginianus...... 9

Chalcopsitta scintilla-

ta ..................... 25

Chauna chavaria ...... 29

Chrysotis agilis ...... 24

albifrons .......... 24

- amazonica ...... 25

- bouqueti .......... 24

finschi ............. 24

- guildingi......... 24

Chub................... 49

Ciconia boyciana...... 28

Circaëtus gallicus ... 27

Civet, Schlegel's ...... 5

Clemmys decussata ... 41 floridana ......... 40

- punctularia....... 41

reevesi ............ 41

- serrata............ 40

— trijuga ......... 41

Z unicolor ............ 41

Cockatoo, Goffin's ... 22

Colobus bicolor......... 1

-, White-thighed. 1

Conure, Red-eared ... 23

-, Wagler's......... 23

Conurus cruentatus... 23

-wagleri............ 23

Coronella phocarum .. 44

Corvus culminatus ... 19

Coryphoden testaceus . 45

Coturnix chinensis ... 31

Coucal Pheasant ...... 21

Crane, White-necked. 34

Crocodile, broad-fron-

ted ................. 42

Crocodilus frontatus. 42 
Crossbill Parrot ...... 16 Crotalus miliarius ... 46 Crow, large-billed ... 19 Crypturus noctivagus 37 obsoletus ......... 37 Cuora amboinensis ... 40 Curlew, Pacific ...... 34 Cyanocorax incas..... 19 Cyanopolius cyaneus 19 Cyanorhamphus alpinus 24

Daption capensis ..... 36 Darter, American ... 28 Dasyprocta antillensis 8 - mexicana........ 8 Deer, Chinese Water. 10 —, Pampas ......... 10 —, Virginian ...... 9 Dendrocitta sinensis.. 20 Dog, Raccoon-like ... 5 Dogfish, large spotted 48 , Picked ........ 48 Dolichonyx oryzivora 18 Dorcopsis luctuosa ... 11 Dove, Greoffroy's ...... 30 _-, Martinican ... 31 - White-winged

Zenaida ............ 31

Dromicia nana......... 11

Duck, Chinese Scaup 29

—, Pink-headed ... 29

- Red-crested

Whistling........... 30

Eagle,MalayanHawk- 27 $\longrightarrow$, Short-toed...... 27 Emberiza chrysophrys 16 Emys europaca ......... 40 Eos riciniata ......... 25

- mbra ............ 25

- tricolor............. 25

Erinaceus collaris ... 4

Eudyptes chrysocome . 37 Euphema chrysogastra 23 Euphonia crassirostris 14 Eupodotis denhami ... 33 Euspiza americana ... 16 - aureola ......... 16 - rutila .............. 16

Falcon, Laughing ... 27 Felis aurata........... 4 - planiceps........ 4 - servalina......... 4 Finch, Cape Palmas . 15 $\longrightarrow$, Grey-necked Serin................... 16 $\longrightarrow$, Red-eyed

Ground. 17
Page

Finch, Yellowish...... 16

Fox, Red-footed ...... 5 , Red-tailed ...... 5

Frog,Changeable Tree 47 Fruit-bat, Formosan . 3 Fuligula mariloides... 29 - rufina ............ 30 Furnarius rufus ...... 20

Gadus merlangus ... 48

- pollachius ...... 48

Gallus stanleyi ...... 32 Garrulax perspicilla.

tus.................... 13

- picticollis......... 13

Gazella arabica ...... 9

- muscatensis...... 9

Gazelle, Arabian ...... 9

- Muscat ......... 9

Gavial ................. 42

Gavialis gangeticus... 42

Grouse, Sharp-tailed. 31

Grus leucauchen ...... 34

Guan, Amazonian ... 32

—, Chattering...... 33

—, Mexican ......... 33

—, White-bellied.. 32

Guillemot, Common . 36

Gull, Audouin's ..... 35

-, Common ...... 35

-, Iceland ......... 35

Halcyon vagans ...... 21

Hapale melanura..... 3

Hangnest, Common 18

-, Orchard......... 19

- Red-rumped ... 18

Hawk, Black-and-

white Sparrow ...- 27

Hedgehog, Collared... 4

Helictis, Orange-bel-

lied ................. 5

Helictis subaurantiaca 5

Hemipode, Lepurana 33

Herpetotheres cachin-

nans ............... 27

Himantopus nigricol-

lis .................... 35

Hinulea whitei......... 44

Homopus areolatus ... 40

Hornbill, Lunated ... 21

-, Malayan......... 21

Howler, Brown ...... 2

Hydropotes inermis... 10

Hyla versicolor......... 47

Ianthonas leucolema . 30

Ibis, Nippon ........ 28

Icterus spurius........ 19

- vulgaris .......... 18
Page

Jacare latirostris...... 42

Jay, Peruvian Blue... 19

Jungle-fowl, Ceylon-

ese................... 32

Kangaroo, Mourning 11

Kestrel, American ... 27

Kingfisher, New-Zea-

land.............. .21

Kiwi..................... 38

Lacerta lilfordi ...... 43

muralis ......... 43

Lamprey, Sea ......... 49

Lancelet ............... 50

Lark, Crested ......... 19

—, Indian Sky ... 17

—, Madras Bush... 18 , White - headed

Bullfinch ........... 18

Larus audouini ...... 35

- canus ............ 35

- leucopterus ....... 35

Lemur, Black-fronted 3 nigrifrons ...... 3

Leptoptilus argala ... 28

Lestris pomatorhinus . 35

Leuciscus cephalus ... 49

Liophis merremii..... 45

Lizard, Knob-nosed.. 43

—, Lilford's......... 43

- Lozenge-mark-

ed ................... 43

, Occipital ..... 42

—, Spanish Sand... 44

—, Spinose ........ 42

$\longrightarrow$, Wall ........... 43

Loriculus asiaticus ... 25

Lorius domicella ...... 25

Lory, Blue-tailed...... 25

—, Green-naped ... 26

- Ornamental ... 26

- Purple-capped. 25

$\longrightarrow$, Red............. 25

— Red-fronted ... 25

—, Violet-necked... 25

Loxia pityopsittacus . 16

Lyriocephalus scuta-

tus................... 43

Macacus brunneus ... 2

—rheso-similis ... 2

- rufescens ......... 2

Macaque, Reddish .... 2

Maccaw, Hahn's ...... 22

Macropygia phasia-

nella ................ 30

Macroscincus cocteauii 44

Magpie, Blue ......... 19

Marmoset,Black-tailed 3 


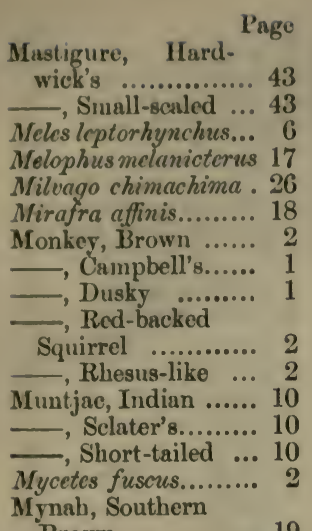

Nestor notabilis ..... 26 Nightjar, Common ... 20 Nothura maculosa ... 37 Numenius femoralis. . 34 Nuthatch, White-bellied

Nyctereutes procynides................... 12 5

Odontophorus dentatus.................... 31 Ortalida albiventris... 32 — garrula ......... 33 - vetula ............. 33

Oryx beatrix ........ 9

beisa ............ 9

Oven-bird, Red ...... 20

Palcornis erythrogenys ................ 23

$\begin{array}{llll}\text { Paroaria capitata ... } 15 & 15\end{array}$

Parrakeet, Alpine .... 24

$\longrightarrow$, CeyloneseHang-

ing ................25

_, Orange-bellied

Grass .............. 23

- Red-cheeked ... 23

- Tabuan ........ 23

- Tovi ............ 23

Parrot, Blue-throated 24

, Mountrin, or

Kea ............... 26

Partridge, Barbary ... 32

—, Capoeira ...... 31

- Gambel's ...... 31

Pusser alario ......... 15

- petronella ....... 15

Parus ater ............ 13

- coruleus .......... 12

- cyanus............ 13
Parus major........... 13

- palustris .......... 13

Penguin, Gentoo...... 36

_, Roek-hopper... 37

Peristera geoffroii ... 30

Petrel, Cape........... 36

Petromyzon marinus . 49

Phalanger, Dormouso 11

Phyllornis aurifrons 14

Picus minor........... 20

Pionus sordidus ..... 24

Pipilo erythrophthal. mus ................. 17

Pigeon, Green Fruit- 30 , Pheasant-tailed 30 , White-throated

Violet .............. 30

Pipile cujubi............. 32

Pituophis catenifer ... 45

Plotus anhinga ..... 28

Plover, Ringed ...... 34

Porphyrio, Indian ... 34

Porphyrio indicus ... 34

Psammodromus hispanicus................. 44

Psammophis elegans... 46

Psophia leucoptera ... 33

Pteroglossus wiedi ... 22

Ptcromys leucogenys . 7

- magnificus ...... 7

Pteropus formosus ... 3

Pueras, Darwin's..... 32

Pucrasia darwini...... 32

Puffinus anglorum ... 36

Pycnonotus hemor-

rhous................. 14

Pygosceles teniatus ... 36

Pyrrhulauda verticalis 18

Pyrrhulopsis tabuensis 23

Pytelia phoenicoptera. 15

Quail, Chinese......... 31

Querquedula falcata . 29

Ramphastos vitellinus 22

Rattlesnake, Smaller . 46

Recurvirostra avocetta 35

Rhinoceros, Hairy-

eared.................. 8

$\longrightarrow$, Javan............. 8

—, Sumatran ...... 8

Rhinoceros lasiotis ... 8

sondaicus......... 8

- sumatrensis...... 8

Rhodonessa caryophyl-

lacea .................. 29

Rhombus lavis......... 49

Rhynchotus perdica-

rius ................. 38

Rice-bird, American . 18
Page

Saimaris arstedi ...... 2

Sciuropterus fimbria-

tus.................. 7

Sciurus erythropus ... 7

- griseopectus...... 6

- macruroides ... 6

- macrurus......... 6

- tennenti .......... 6

- tristriatus ....... 6

Screamer, Crested .... 29

Scyllium stellare ...... 48

Sea-Lamprey ........ 49

Semnopithecus obscu-

rus ................ 1

Seps, Three-tood...... 44

Seps tridactylus ...... 44

Serinus canicollis ... 16

Shearwater, Manx ... 36

Sitta europea ......... 12

Skink, Cocteau's ..... 44

—, White's ......... 44

Skua, Pomatorhine... 35

Snake, Chained ..... 45

-, Lineated ...... 45

—, Merrem's ...... 45

- Robben-Island . 44

—, Seven-banded .. 45

-, Slender Sand- . 46

_. Testaceous ...... 45

Souslik, Mongolian... 7

Sparrow, Alaric ...... 15

-, Pileated Song . 17

, White-throated

Song................. 17

- Yellow-throated 15

Spermophilus mongo-

licus ................. 7

Spizaëtus caligatus ... 27

Squirrel, Black Hill . 6

-, Chipping ..... 7

—, Grey-breasted . 6

—, Grey Flying ... 7

$\longrightarrow$ Grizzled Hill... 6

_, Red-bellied Fly-

ing ................ 7

$\longrightarrow$ Tennent's ........ 6

—, Three-striped.. 6

-, White-banded . 7

-, White-cheeked

Flying .............. 7

Starling, Chopi ..... 19

Sterna cantiaca ...... 36

Stork, Boyee's ......... 28

Struthidea cinerea ... 13

Struthidea, Grey ...... 13

Stilt, Black-necked ... 35

Sycalis luteola ........ 16

Sylvia atricapilla ... 12 


\begin{tabular}{|c|c|c|}
\hline Page & Page & Page \\
\hline Tamias striatus ...... 7 & Tit, Blue .............. i2 & Uria troile ........ \\
\hline Tanager, Black-shoul- & $\longrightarrow$, Cole ............ 13 & Uromastix hardwickii 43 \\
\hline , Blue-and-black 14 & $\begin{array}{l}- \text {, Marsh ............ } 13 \\
\end{array}$ & Urubitinga anthraci- \\
\hline Thick-billed & Titmouse, Azure ...... 13 & $n a \ldots \ldots \ldots \ldots \ldots \ldots . . . .27$ \\
\hline $\begin{array}{cr}\text { Violet .............. } 14 \\
\text { Taphozous nudiventer }\end{array}$ & Toad, Giant............ 47 & \\
\hline $\begin{array}{l}\text { Taphozous nudiventer } 4 \\
\text { Teal, Falcated ......... } 29\end{array}$ & Italian Fire- & Vespertilio nattereri. 3 \\
\hline $\begin{array}{l}\text { Teal, Falcated ......... } \\
\text { Term, Sandwich ...... } 39\end{array}$ & bellied. .............. 47 & Viverricula schlegeli. \\
\hline $\begin{array}{l}\text { Tern, Sandwich ...... } 36 \\
\text { Terrapin, Black-head- }\end{array}$ & $\begin{array}{c}\text { Tortoise, Algerian ... } 39 \\
\\
\end{array}$ & \\
\hline ed .................. & , Areolated ...... 40 & Waxbill, Crimson- \\
\hline 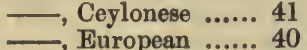 & —, Burmese........ 40 & winged .............. \\
\hline $\begin{array}{l}\text { European } \ldots . . .440 \\
\text { Floridan ..... } 40\end{array}$ & -, semiserrated ... 39 & Weever, Lesse \\
\hline $\begin{array}{l}\text { Floridan } . . . . . \\
\text { Hicotee } . . . . . . .\end{array}$ & ..... 39 & White-eye, Lateral ... \\
\hline $\begin{array}{l}\text { Hicotee .......... } \\
\text { Reeve's ......... }\end{array}$ & oucan, Sulphu & 10n \\
\hline Rough .......... 41 & Trachinus vipera...... 48 & 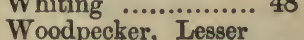 \\
\hline -, Serrated......... 40 & Tree-Pie, Chinese ... 20 & Spotted................ \\
\hline 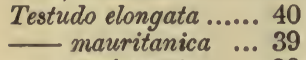 & $\begin{array}{l}\text { Trichoglossus cyano- } \\
\text { grammus ............. } 26\end{array}$ & Wryneck .... \\
\hline $\begin{array}{l}\text { - semiserrata ...... } 39 \\
\\
\text { stellata }\end{array}$ & 7 ornatus ........ 26 & Xanthosomus flavus... \\
\hline $\begin{array}{l}\text { stellata .......... } \\
\text { to phasianellus... }\end{array}$ & Triony $x$ ferox ........ 41 & Xenus unicinctus...... \\
\hline sh, Collared Jay 13 & Trionyx, North-Ame- & \\
\hline 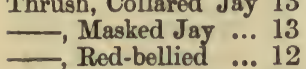 & $\begin{array}{l}\text { rican } \\
\text { Tropidonotus le......... } 41\end{array}$ & Yunx torquilla. \\
\hline $\begin{array}{ccc}- \text {, Red-bellied ... } & 12 \\
\text { namou, Banded } & . . & 37\end{array}$ & Trumpeter, White- & Zamenis caudilinea- \\
\hline Chilian ......... 3 & & \\
\hline Obsolete........... 37 & $\begin{array}{l}\text { Trupial, Yellow } \\
\text { Turdus rufiventris .... } 18\end{array}$ & $\begin{array}{l}\text { Zenaida leucoptera ... } \\
\text { L martinicana }\end{array}$ \\
\hline Solitar & Turnix lepura & Zonotrichia albicollis 1 \\
\hline & atted... 30 & \\
\hline Tinamus solitarius ... 37 & Turtur suratensis ... 30 & Zosterops lateralis ... \\
\hline
\end{tabular}

THE END.

Printed by Taylor and Fraxcis, Red Lion Court, Fleet Street. 



\title{
L I S T
}

or

\section{THE VERTEBRATED ANIMALS}

NOW OR LATELY LIVING

\section{IN THE GARDENS}

OF THE

\section{Z00LOGICAL SOCIETY OF LONDON.}

\author{
FIRST SUPPLEM N T, \\ CONTAINING ADDITIONS RECEIVED IN \\ 1879.
}

PRINTED FOR THE SOCIETY, AND SOLD AT THEIR HOUSE IN HANOVER SQUARE. L ONDON : MESSRS. LONGMANS, GREEN, READER, AND DYER, PATERNOSTER ROW. 


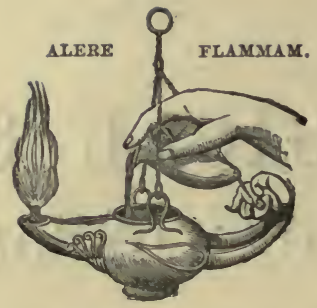

PRINTED BY TAYLOR AND FRANCIS, RED LION COURT, FLEET STREET. 


\section{PREFACE.}

The present Supplement contains the names of the Vertebrated Animals received by the Society in 1879, arranged according to the last edition of the List of Animals (1879), together with the date of the arrival and mode of acquisition of each specimen.

The numbers are the same as those attached to each species in the List.

The additional species are indicated by an asterisk, and their places in the series indicated by the number followed by a letter $(8 a \& c$.$) .$

The total number of additions to the Society's Menagerie in 1879 was 1268, belonging to 491 species. Of these species the following 50 were new to the collection :-

\section{MAMNALS.}

Semnopithecus mitratus.

Brachyurus rubicundus.

Felis caffra.

Canis rudis.

Icticyon renaticus.

Martes foina.
Lutra nair.

Gerbillus indicus.

Lepus brasiliensis.

Capricornus crispus.

Capreolus pygargus.

Cariacus macrotis. 


\section{BIRDS.}

Accentor collaris.

Phyllornis hardwicki.

Tachyphonus coronatus.

Pyranga saira.

Pytelia wieneri.

Emberiza citrinella.

Eremophila chrysolæma.

Garrulus lanceolatus.

Cyanocorax cyanomelas.

Corythaix porphyreolophus.

Selenidera maculirostris.

Calyptorhynchus funereus.

Nymphicus cornutus.
Conurus auricapillus.

Chrysotis bodini.

Chrysotis erythrura.

Chrysotis coligena.

Brotogerys tuipara.

Ardea russata.

Ardetta involucris.

P'hlogœnas stairi.

Numida ellioti.

Rallus maculatus.

Rallus rhythirhynchus.

Rallina pociloptera.

Colymbus septentrionalis.

\section{REPTILES.}

Amphisbæna darwini. Phyllodactylus europæus.

Taraguira smithi.

Crotaphytus wislizeni. Acontias meleagris.
Tortrix scytale.

Rhinostoma cupreum.

Rhinechis scalaris.

Liophis cobella.

Geoptyas collaris.

\section{FISHES.}

Huro nigricans.

Fundulus majalis.

P. L. S.

11 Hanorer Square, W.,

1st March, 1880. 


\section{CONTENTS.}

MAMMLLIA $\ldots \ldots \ldots \ldots \ldots \ldots \ldots \ldots$ page 1

Quadrumana..page $1 \mid$ Hyraces .......... 14

Lemures ...... $6 \quad$ Ungulata .......... 14

Chiroptera ..... 7 Edentata ......... 18

Carnirora ....... 7 Marsupialia ....... 19

Rodentia ....... 12

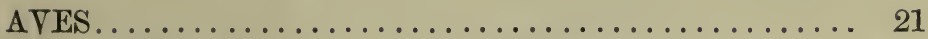

Passeres ...... $21 \mid$ Columbæ ......... 45

Cypseli........ 28 Gallinæ.......... 47

Pici ........ $28 \quad$ Fulicariæ ......... 50

Coccyges ...... $28 \quad$ Alectorides ....... 51

Psittaci........ $29 \quad$ Limicolæ ......... 52

Striges ....... 34 Gaviæ ......... 54

Accipitres...... 35 Pygopodes ....... 55

Steganopodes .... 38 Impennes ......... 55

Herodiones ..... 39 Crypturi ......... 55

Anseres ...... 40 Struthiones ....... 55

REPTILIA ......................... 56

Testudinata .... $56 \quad$ Sauria .......... 57

Crocodilia...... $56 \quad$ Ophidia ........ 59

AMPHIBIA $\ldots \ldots \ldots \ldots \ldots \ldots \ldots \ldots \ldots \ldots \ldots \ldots \ldots \ldots \ldots$

Batrachia ......6 63 | Urodela ........ 63

PISCES ......................... 64 


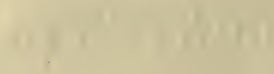

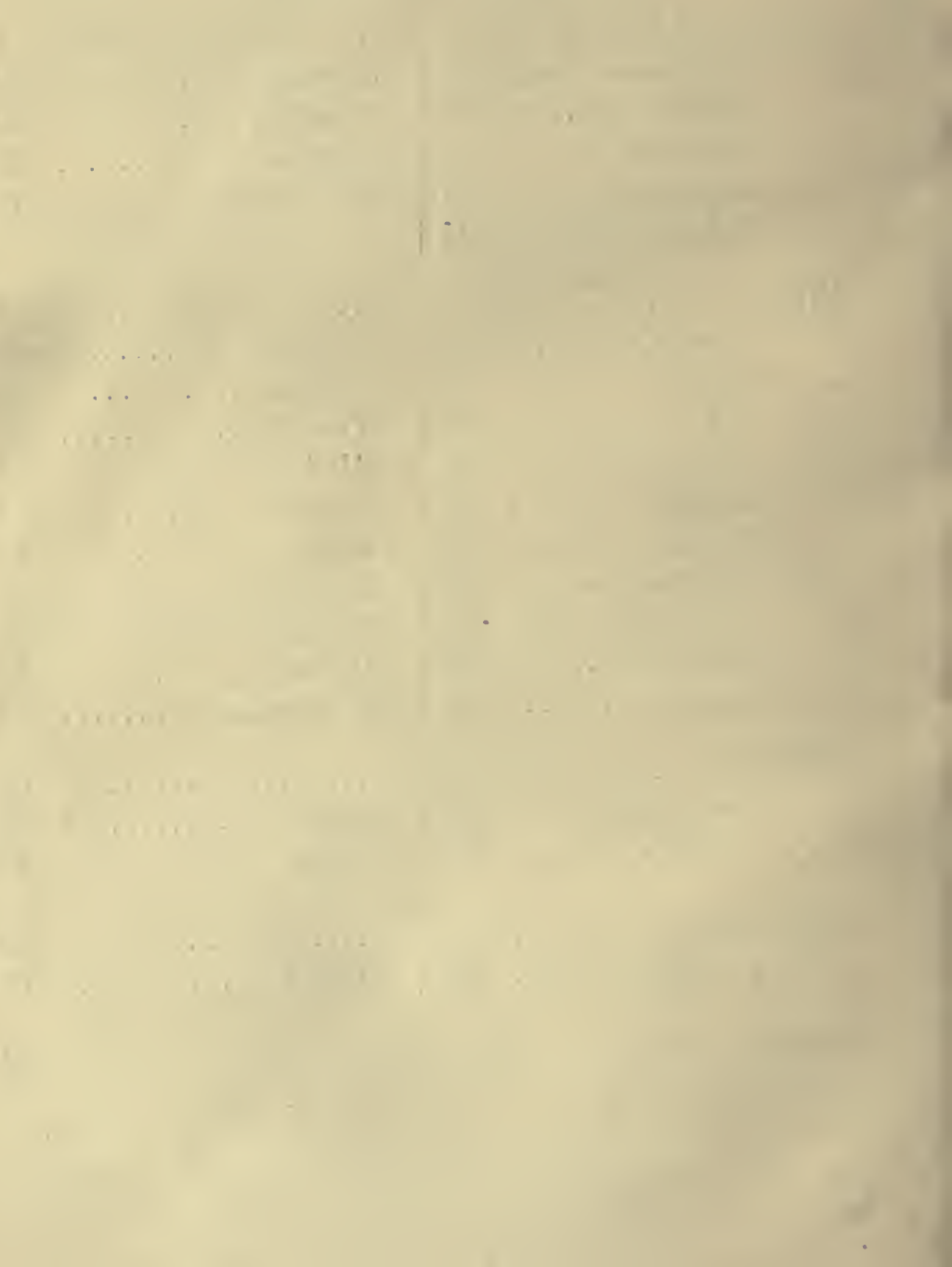




\section{LIST OF ANIMALS}

RECEIVED IN 1879.

\section{Class MAMMALIA. \\ Order QUADRUMANA.}

8. Semnopithecus entellus (Dufresne). Lntellus Monkey. $H a b$. India.

n. Presented by J. Mills, Esq., R.H.A., Jan. 23, 1879.

o. Female. Received in exchange, April 12, 1879.

*8a. Semnopithecus mitratus, Eschsch. Mitred Monkey. Hab. Java.

a. Received in exchange, March 12, 1879.

14. Colobus bicolor (Wesmael). White-thighed Colobus. $H a b$. West Africa.

d. Presented by Dr. W. H. Hart, May 27, 1879.

16. Cercopithecus cynosurus (Scop.). Malbrouck Monkey. Hab. West Africa.

s. Female. Presented by Miss Agnes Barker, Aug. 15, 1879.

t. Male. Purchased, Sept. 23, 1879.

u. Female. Deposited, Nov. 7, 1879.

v. Male. Presented by the Rev. J. L. Sabunjie, Dec. 17, 1879.

17. Cercopithecus lalandii, Is. Geoffr. Vervet Monkey. $H a b$. South Africa.

$p^{2}$. Presented by W. T. Millar, Esq., Aug. 25, 1879.

$q^{2}$. Presented by E. Meyerstein, Esq., Sept. 25, 1879. 
$r^{2}$. Presented by Sir Arthur Scott, Bart., Oct. 4, 1879.

$s^{2}$. Male. Deposited, Nov. 4, 1879.

18. Cercopithecus griseo-viridis, Desm. Grivet Monkey. Hab. North-east Africa.

u. Presented by W. B. Greenfield, Esq., April 8, 1879.

v. Male. Presented by R. M. Courage, Esq., July 1, 1879.

19. Cercopithecus callitrichus, Is. Geoffr. Green Monkey. Hab. West Africa.

$h^{2}$. Female. Presented by Mr. Carroll, Jan. 10, 1879.

$i^{2}$. Presented by J. Douglas Murray, Esq., Feb. 21, 1879.

$j^{2}$. Presented by Miss E. A. B. Payton, March 4, 1879.

$k^{2}$. Male. Presented by J. N. T. Marthege, Esq., March 28, 1879.

$l^{2}$. Male. Presented by C. F. S. Day, Esq., Aug. 15, 1879.

24. Cercopithecus mona (Schreb.). Mona Monkey. $H a b$. West Africa.

r. Female. Presented by Miss Sandford, March 22, 1879.

s. Female. Deposited, Nov. 7, 1879.

25. Cercopithecus diana (Linn.). Diana Monkey. Hab. West Africa.

n. Female. Received in exchange, May 8, 1879.

$0, p$. Female. Presented by F. J. Crocker, Esq., Aug. 14, 1878.

30. Cercopithecus cephus (Linn.). Moustache Monkey. Hab. West Africa.

$b^{1}, c^{1}$. Purchased, Nov. 6, 1879.

32. Cercopithecus petaurista (Schreb.). Lesser White-nosed Monkey.

Hab. West Africa.

m. Male. Presented by R. F. Clothier, Esq., July 15, 1879.

37. Cercocebus albigena (Gray). Grey-cheeked Monkey. Hab. West Africa.

j. Female. Presented by Robert Surrey, Esq., May 20, 1879. 
38. Macacus radiatus (Shaw). Bonnet-Monkey. Hab. Continental India.

9. Male. Presented by Geo. Eggar, Esq., March 18, 1879.

$h^{5}$. Male. Presented by Mr. E. Brett, May 1, 1879.

$i^{5}$. Female. Presented by Mrs. J. Raves, Sept. 4, 1879.

$j^{5}$. Female. Presented by Mrs. Bonamy Dobree, Oct. 18, 1879.

$k^{5}$. Presented by S. E. Phillips, Esq., Oct. 20, 1879.

7. Male. Presented by J. N. Medley, Esq., Oct. 23, 1879. $m^{5}$. Female. Presented by H. L. Ruegg, Esq., Nov. 14, 1879. $n^{5}$. Male. Presented by the Rev. E. C. Ince, Dec. 12, 1879. $0^{5}$. Presented by R. M. Drury, Esq., Dec. 17, 1879.

-40. Macacus cynomolgus (Linn.). Macaque Monkey. $H a b$. India.

$d^{7}$. Female. Presented by Mr. W. Trent, Jan. 9, 1879.

$e^{7}$. Female. Presented by D. D'Orpen, Esq., Jan. 14, 1879.

$f^{7}$. Male. Presented by E. E. Barclay, Esq., Jan. 27, 1879.

$g^{7}$. Female. Presented by Mrs. Eardley Holt, Jan. 30, 1879.

$h^{7}$. Male. Presented by C. A. Thomson, Esq., May 5, 1879.

$i^{7}$. Female. Presented by F. V. Goddard, Esq., May 5, 1879.

$7^{7}$. Female. Presented by P. Leman, Esq., May 10, 1879.

$k^{7}$. Male. Presented by Mr. G. T. Close, June 19, 1879.

$l^{7}$. Presented by Miss E. Catlin, June 20, 1879.

$\mathrm{m}^{7}$. Male. Presented by Mrs. James Raves, Sept. 4, 1879.

$n^{7}$. liemale. Presented by W. Leckie, Esq., Oct. 7, 1879.

$o^{7}$. Presented by T. Hobbs, Esq., Oct. 14, 1879.

$p^{7}$. Male. Presented by Mrs. Franklin, Oct. 21, 1879.

$q^{7}$. Male. Deposited, Nov. 25, 1879.

$r^{7}$. Female. Presented by Mrs. L. C. Piggott, Dec. 30, 1879.

41. Macacus nemestrinus (Linn.). Pig-tailed Monkey. Hab. Java.

z. Female. Presented by Mrs. J. E. Fenton, March 7, 1879. $a^{1}$. Female. Presented by E. M. Clissold, Esq., May 2, 1879.

$b^{1}$. Female. Presented by Capt. A. W. Shean, May 10, 1879.

$c^{1}$. Presented by Mrs. Walter Crane, Oct. 2, 1879.

44. Macacus erythraus (Schreb.). Rhesus Monkey. $H a b$. Continental India.

$q^{3}$. Presented by Mr. J. Roberts, April 8, 1879.

$r^{3}$. Presented by H. Winsor, Esq., May 12, 1879.

$s^{3}$. Presented by J. Beech, Esq., June 3, 1879.

$t^{3}$. Female. Deposited, June 11, 1879.

$u^{3}$. Female. Presented by Jas. Bartle, Esq., July 4, 1879.

$v^{3}$. Female. Presented by Dr. Douglas, Oct. 17, 1879. 
$w^{3}$. Female. Presented by R. C. Bonsfield, Esq., Oct. 17, 1879.

$x^{3}$. Presented by T. G. Anderson, Nov. 8, 1879.

$y^{3}$. Female. Presented by F. J. Lightfoot, Esq., Dec. 11, 1879.

60. Cynocephalus porcarius (Bodd.). Chacma Baboon.

$H a b$. South Africa.

s. Deposited, Aug. 18, 1879.

t. Deposited, Sept. 18, 1879.

u. Male. Deposited, Oct. 7, 1879.

62. Cynocephalus babouin, Desm. Yellow Baboon. Hab. West Africa.

l. Deposited, Sept. 18, 1879.

m. Female. Presented by Cecil B. Hankey, Esq., Nov. 20, 1879.

63. Cynocephalus sphinx (Linn.). Guinea Baboon. Hab. West Africa.

o. Presented by P. Lambery, Esq., Aug. 19, 1879.

p. Presented by F. Naylor, Esq., Sept. 12, 1879.

68. Ateles ater, F. Cuv. Black-faced Spider Monkey. Hab. Eastern Peru.

$u^{1}$. Presented by the Earl Brownlow, F.Z.S., Jan. 15, 1879.

$v^{1}$. Received in exchange, March 12, 1879.

$w^{1}$. Male. Presented by Capt. H. King, May 2, 1879.

$x^{1}$. Deposited, June 30, 1879.

$y^{1}$. Presented by Capt. H. King, Sept. 8, 1879.

74. Ateles geoffroyi (Kuhl). Black-handed Spider Monkey. $H a b$. Central America.

$h^{1}$. Received in exchange, March 12, 1879.

$i^{1}$. Presented by D. R. Comyn, Esq., April 24, 1879.

80. Cebus lunatus (F. Cuv.). White-cheeked Capuchin. Hab. Brazil.

j. Male. Presented by Adrian Hope, Esq., Oct. 4, 1879. 
81. Cebus capucinus, Geoffr. Weeper Capuchin. Hab. Brazil.

$r$. Presented by W. Fridrick, Esq., Jan. 29, 1879.

s. Male. Presented by Capt. R. Bond, July 28, 1879.

$t$. Female. Presented by A. Sargent, Esq., Oct. 28, 1879.

82. Cebus albifrons, Geoffr. White-fronted Capuchin Monkey. Hab. South America.

v. Presented by Major H. L. Gleig, Sept. 14, 1879.

83. Cebus hypoleucus (Humb.). White-throated Capuchin. Hab. Central America.

$n^{1}, n^{1}$. Presented by James Backhouse, Esq., Jan. 21, 1879.

88. Chrysothrix sciurea (Linn.). Squirrel Monkey.

Hab. Guiana.

$c^{1}, d^{1}$. Males. Purchased, May 17, 1879.

*95a. Brachyurus rubicundus (Geoffr. et Dev.). Red-faced Saki.

Hab. Upper Amazons.

a. Purchased, May 24, 1879. See P.Z. S. 1879, p. 551.

96. Hapale jacchus (Linn.). Common Marmoset.

$H a b$. South-east Brazil.

$t^{2}$. Presented by Mrs. Currey, Jan. 10, 1879.

97. Hapale penicillata (Geoffr.). Black-eared Marmoset.

Hab. South-east Brazil.

$l^{1}$. Purchased, June 14, 1879.

102. Midas ursulus (Hoffm.). Negro Tamarin.

Hab. Guiana.

h. Purchased, June 14, 1879.

104. Midas geoffroii, Puch. Geoffroy's Marmoset. Hab. Panama.

h. Purchased, July 22, 1879. 
105. Midas rosalia (Linn.). Silky Marmoset. Hab. South-east Brazil.

$b^{1}, c^{1}$. Presented by Mrs. Hector, May 26, 1879

(7). Male. Deposited, Sept. 22, 1879.

\section{Order LEMURES.}

113. Lemur rufifrons. Red-fronted Lemur. Hab. Madagascar.

e. Male. Received in exchange, Aug. 4, 1879.

115. Lemur catta, Linn* Ring-tailed Lemur.

Hab. Madagascar.

i. Male. Preșented by Thomas G. Mann, Esq., Feb. 11, 1879.

j. Male; k. Female. Presented by Hugh McCubbin, Esq., July 15, 1879.

l. Female. Presented by F. E. Colenso, Esq., Dec. 12, 1879.

119. Nycticebus tardigradus (Linn.). Slow Loris. Hab. Malay countries, Sumatra and Borneo. $u, v$. Purchased, Aug. 27, 1879.

$x$. Purchased, Dec. 1, 1879.

121. Loris gracilis, Geoffr. - Slender Loris.

Hab. Ceylon.

d, e. Presented by Leith Bonhôte, Esq., Feb. 5, 1879.

122. Perodicticus potto (Gm.). Bosman's Potto.

$H a b$. West Africa.

i. Purchased, Oct. 14, 1879.

j. Purchased, Oct. 28, 1879.

123. Galago crassicaudata (Geoffr.). Grand Galago. Hab. East Africa.

$f, g$. Purchased, Feb. 22, 1879.

h. Presented by Mr. W. Jenkins, June 30, 1879. 
125. Galago garnetti (Ogilby). Garnett's Galago.

$H a b$. Eastern Africa.

k. Purchased, Feb. 12, 1879.

l. Presented by F. W. Barff, Esq., June 21, 1879.

m. Purchased, Oct. 24, 1879.

\section{Order CHIROPTERA.}

130. Pteropus medius, Temm. Indian Fruit-Bat. $H a b$. India.

i. Presented by Capt. F. P. Millett, March 10, 1879 .

134. Cynonycteris collaris (Illiger). Collared Fruit-Bat. $H a b$. South Africa.

u. Born in the Menagerie, June 21, 1879.

v. Purchased, July 4, 1879. Captured on the Red Sea.

137. Plecotus auritus (Linn.). Long-eared Bat.

Hab: Europe.

b. Presented by Mr. J. Ward, A pril 24, 1879.

\section{Order CARNIVORA.}

145. Felis tigris, Linn. Tiger.

Hab. Eastern Asia.

$n^{1}$. Female. Presented by H. E. the Right Hon. Lord Lytton, G.C.B., C.M.S.I., Sept. 4, 1879.

146. Felis pardus, Linn. Leopard.

$H a b$. Southern Asia and Africa.

$z^{1}, a^{2}$. Presented by H. E. the Right Hon. Lord Lytton, G.C.B., C.M.S.I., Sept. $4, \cdot 1879$.

148. Felis jubata, Schreb. Cheetah.

Hab. Africa and South-west Asia.

i. Female. Purchased, May 24, 1879. 
*160a. Felis caffra (Desm.). Caffer Wild Cat. Hab. S. Africa.

a. Presented by R. Southey, Esq., Oct. 31, 1879.

163. Felis caracal, Schreb. Caracal.

Hab. South Asia and Africa.

g. Presented by Dr. Holub, Sept. 18, 1879.

164. Felis concolor, Linn. Puma.

$\mathrm{Hab}$. America.

n. Male. Purchased, May 14, 1879.

o. Male. Presented by Lord Lilford, F.Z.S., June 30, 1879.

168. Felis pardalis, Linn. Ocelot.

Hab. America.

$t^{1}$. Presented by B. H. Jones, Esq., May 1, 1879.

$u^{1}$. Presented by P. Leckie, Esq., May 6, 1879.

$v^{1}$. Purchased, July 15, 1879.

$w^{1}$. Male. Purchased, Dec. 31, 1879.

169. Felis geoffroii, D'Orb. et Gerv. Geoffroy's Cat. Hab. Paraguay and La Plata.

d, e. Deposited by Lord Lilford, Nor. 21, 1879.

175. Hyana brunnea, Thunb. Brown Hyæna.

$H a b$. South Africa.

c. Purchased, May 30, 1879.

176. Hyæna striata, Zimm. Striped Hyæna.

$H a b$. India.

j. Purchased, July 12, 1879.

178. Viverra civetta (Schreb.). African Civet Cat. Hab. Africa.

$m, n$. Presented by M. B. Salmon, Esq., June 20, 1879.

185. Paradoxurus typus, F. Cuv. Common Paradoxure. Hab. India. $x, y$. Presented by G. K. Lloyd, Esq., June 11, 1879. 
192. Paradoxurus leucomystax, Gray. White-whiskered Paradoxure.

$H a b$. East India.

o. Presented by Mr. Carl Bock, June 10, 1879.

201. Herpestes griseus (Geoffr.). Grey Ichneumon. Hab. India.

$e^{2}$. Presented by B. Baverstock, Esq., Jan. 27, 1879.

204. Herpestes auropunctatus, Hodgs. Spotted Ichneumon. Hab. Nepal.

n. Presented by Miss H. Boteler, March 16, 1879.

205. Herpestes fasciatus, Desm. Banded Ichneumon. Hab. West Africa.

o. Purchased, Uct. 24, 1879.

p. Presented by H. L. Cocksedge, Esq., Nov. 15, 1879.

214. Canis rulpes, Linn. Common Fox. $H a b$. British Islands.

$f^{1}$. Presented by James Wheatley, Esq., Sept. 19, 1879.

220. Canis aureus, Linn. Common Jackal.

$H a b$. India.

o. Presented by T. Thursfield, Esq., Sept. 8, 1879.

222. Canis mesomelas, Schreb. Black-backed Jackal. Hab. South Africa.

q. Presented by E. J. Redman, Esq., April 1, 1879.

230. Canis fulvus, Desm. Red Fox.

Hab. North America.

q. Presented by Lord Hobart, Oct. 4, 1879. From Labrador.

*233a. Canis rudis, Günther. Rude Fox.

$H a b$. Demerara.

a. Deposited, April 6, 1879. 
b. Presented by G. H. Hawtayne, Esq., C.M.Z.S., Aug. 5, 1879. See Ann. \& Mag. Nat. Hist. ser. 5, vol. i. p. 316 (1879).

*240a. Icticyon venaticus, Lund. Bush-Dog.

$H a b$. British Guiana.

a. Presented by J. Ernest Tinné, Esq., Aug. 20, $1879 . \quad$ See P.Z. S. 1879 , p. 664.

245a. Martes foina (Erxl.). Beech-Marten.

Hab. Russia.

a. Purchased, June 17, 1879.

249. Mustela vulgaris (Gm.). Weasel.

Hab. British Isles.

b. Purchased, Nov. 3, 1879.

251. Galictis barbara (Linn.). Tayra.

Hab. South America.

j. Purchased, Feb. 17, 1879.

258. Meles taxus (Schreb.). Common Badger.

$H a b$. British Islands.

$r-t$. Born in the Menagerie, March 10, 1879.

263. Conepatus mapurito (Gmel.). Chilian Skunk. Hob. Chili.

d, e. Deposited, March 6, 1879.

*264a. Lutra nair, F. Cuv. Indian Otter.

$H a b$. India.

a. Purchased, Aug. 27, 1879. From Rangoon. See P.Z. S. 1879, p. 664 .

266. Procyon cancrivorus (Cuv.): Crab-eating Raccoon. $H a b$. South America.

j. Presented by R. Bridgett, Esq., April 30, 1879. 
268. Nasua rufa (Desm.). Ring-tailed Coati.

$H a b$. South America.

$u^{1}$. Purchased Jan. 24, 1879. From Buenos Ayres.

$v^{2}$. Purchased, March 7, 1879.

$w^{1}$. Presented by C. S. Barnes, Esq., Sept. 17, 1879. Paraguay.

$x^{\mathrm{t}}$. Presented by Percy Brewis, Esq., Sept. 19, 1879.

269. Cercoleptes caudivolvulus (Pall.). Kinkajou.

Hab. Demerara.

$j^{2}$. Presented by M. B. Salmon, Esq., June 20, 1879.

272. Ursus arctos, Linn. Brown Bear.

Hab. Northern Europe and Asia.

v. Presented by J. R. Boyce, Esq., Aug. 2, 1879.

w. Male. Received in exchange, Sept. 22, 1879.

274. Ursus syriacus, Ehrenb. Syrian Bear.

Hab. Western Asia.

d. Presented by Dr. J. Huntley, March 3, 1879.

279. Ursus americanus, Pall. Black Bear.

$H a b$. North America.

q. Male. Received in exchange, June 12, 1879.

283. Otaria jubata (Gm.). Patagonian Sea-Lion.

$H a b$. Antarctic America.

c. Male. Presented by F. E. Cobb, Esq., May 20, 1879. See P.Z. S. 1879, p. 551.

286. Phoca vitulina, Linn. Common Seal.

Hab. British seas.

$f^{1}$. Presented by the Earl of Hopetoun, Jan. 15, 1879.

$g^{1}$. Presented by Capt. Charles Rawle, April 8, 1879.

$h^{1}$. Purchased, June 7, 1879. 


\section{Order RODENTIA.}

299. Sciurus plantani, Ljungh. Plantain-Squirrel. Hab. Java.

h. Male; $i$. Female. Purchased, May 17, 1879.

j. Presented by Miss Lizzie Casey, Sept. 10, 1879.

308. Sciurus vulpinus, Gm. Vulpine Squirrel. Hab. North America.

e. Purchased, Sept. 8, 1879. Var. capistrata.

324. Sciuropterus fimbriatus, Gray. Grey Flying Squirrel. Hab. Northern India.

b. Presented by Mrs. Louisa Edwards, Aug. 8, 1879.

329. Arctomys monax (Linn.). Quebec Marmot. Hab. North America.

n. Purchased, Nov. 8, 1879.

330. Arctomys caudatus, Jacq. Long-tailed Marmot. Hab. Bhootan.

c. Presented by the Hon. Sir Ashley Eden, K.C.S.I., Apr. 10, 1879.

d. Presented by Capt. Greenstreet, R.E., May 2, 1879.

331. Cynomys ludovicianus (Ord). Prairie-Marmot. Hab. North America.

n, o. Presented by Miss Agneta B. Dykes, Jan. 23, 1879.

$p, q$. Received in exchange, Feb. 18, 1879.

r, s. Presented by W. G. Marshall, Esq., April 23, 1879.

332. Castor canadensis, Kuhl. Canadian Beaver.

Hab. North America.

$d^{1}$. Born in the Menagerie, June 8, 1876.

333. Myoxus glis (Linn.). Fat Dormouse.

Hab. Europe.

$j-n$. Presented by Edwin Liot, Esq., Oct. 5, 1879. From Serrach, near Esslingen, Würtemberg. 
336a. Gerbillus indicus (Hardwicke). Indian Jerboa Rat. $H a b$. India.

$a-h$. Purchased, March 31, 1879.

337. Mus rattus, Linn. Black Rat.

Hab. British Islands.

$w, y$. Born in the Menagerie, June 23, 1879.

z. Presented by R. M. Middleton, Esq., Sept. 26, 1879.

347. Myodes lemmus (Linn.). Norwegian Lemming.

Hab. Norway.

h. Presented by James Shuter, Esq., Sept. 25, 1879.

356. Myopotamus coypus (Mol.). Coypu.

$\mathrm{Hab}$. South America.

$k, l$. Purchased, Jan. 24, 1879. From Buenos Ayres.

357. Octodon cumingi, Benn. Cuming's Octodon.

$\mathrm{Hab}$. Chili.

$a^{2}, b^{2}$. Born in the Menagerie, Jan. 13, 1879.

$c^{2}, d^{2}$. Deposited, Jan. 21, 1879 .

358. Hystrix cristata (Linn.). Crested Porcupine.

Hab. Asia and Africa.

$g^{1}, h^{1}$. Presented by Moses S. Boyle, Esq., July 9, 1879.

361. Atherura africana, Gray. African Brush-tailed Porcupine.

Hab. West Africa.

e. Purchased, Sept. 8, 1879.

367. Chinchilla lanigera, Benn. Chinchilla.

Hab. Chili.

x. Presented by Sir Charles Smith, March 18, 1879.

$y, z$. Born in the Menagerie, May 21, 1879.

370. Dasyprocta isthmica, Alston. Central-American Agouti. Hab. Central America.

$u^{1}$. Purchased, July 29, 1879. 
377. Dasyprocta prymnolopha, Wagl. Hairy-rumped Agouti. Hab. Guiana.

k, l. Born in the Gardens, Feb. 1, 1878.

378. Dasyprocta acouchy (Erxl.). Acouchy. Hab. British Guiana.

$e, f$. Purchased, March 7, 1879.

382. Cerodon mupestris (Max.). Rock-Cavy. Hab. Brazil.

o. Purchased, Sept. 3, 1879.

383. Hydrochœrus capybara, Erxl. Capybara. Hab. South America.

r. Presented by H. B. Whitmarsh, Esq., May 31, 1879.

385. Lepus europæus, Pallas. Common Hare. Hab. British Islands.

t. Presented by Mr. A. Withers, Feb. 17, 1879.

u. Presented by Mrs. Buckland, Mar. 1, 1879.

*386a. Lepus brasiliensis, Linn. Brazilian Hare. Hab. Brazil.

a. Purchased, March 29, 1879.

\section{Order HYRACES.}

391. Hyrax capensis, Schreb. Cape-Hyrax. $H a b$. South Africa.

p. Presented by Mr. A. H. Jamrach, Feb. 12, 1879.

\section{Order UNGULATA.}

395. Rhinoceros sumatrensis, Cuvier. Sumatran Rhinoceros. Hab. Malacca.

d. Male. Deposited, March 20, 1879. Died April 6, 1879. See P.Z.S. 1879 , p. 308. 
416. Sus leucomystax, Temm. White-whiskered Swine.

Hab. Japan.

d. Male. Presented by Theodore Hance, Esq., C.M.Z.S., Aug. 6, 1879. From China.

419. Potamochorus penicillatus (Schinz). Red River-Hog.

Hab. West Africa.

g, h. Females. Deposited, Dec. 4, 1879.

421. Dicotyles tajaçu (Linn.). Collared Peccary.

$H a b$. South America.

s. Presented by H. Sandbach, Esq., Nov. 22, 1879.

432. Camelus bactrianus, Linn. Bactrian Camel.

$H a b$. Central Asia.

c. Female ; d. Female (juv.). Deposited, May 19, 1879.

433. Bos indicus, Linn. Zebu.

Hab. Hindostan.

w. Male. Born in the Menagerie, May 16, 1879.

437. Poëphagus grunniens (Linn.). Yak.

Hab. Tibet.

j. Male. Presented by the Hon. Sir Ashley Eden, K.C.S.I., April 10, 1879.

441. Bubalus caffer (Sparrm.). Cape Buffalo.

$H a b$. South Africa.

i. Born in the Menagerie, Aug. 21, 1879.

444. Oreas canna (H. Smith). Eland.

$H a b$. South Africa.

$i^{\prime}$. Male. Born in the Menagerie, June 3, 1879.

455. Gazella dorcas (Linn.). Gazelle.

Hab. Egypt.

p. Male; $q$. Female. Presented by Commander J. Pratt, June $17,1879$. 
458. Gazella arabica (Hempr. et Ehr.). Arabian Gazelle. Hab. Arabia.

j, k. Females. Presented by Capt. W. Bowden Smith, R.N., Oct. $13,1879$.

461. Gazella subgutturosa (Güldenst.). Persian Gazelle. $H a b$. Persia.

r. Male. Presented by C. H. Watts, Esq., June 19, 1879.

463. Gazella sœmmerringi (Cretzschm.). Sœmmerring's Gazelle.

Hab. Abyssinia.

g. Female. Purchased, Sept. 5, 1879.

h. Male. Purchased, Oct. 20, 1879.

467. Antilope cervicapra (Linn.). Indian Antelope.

$H a b$. India.

l. Male. Presented by the Hon. A. Greville, June 13, 1879.

468. Antilocapra americana (Ord). Pronghorn Autelope. Hab. North America.

c. Male; d. Female. Purchased, Dec. 4, 1879.

469. Cervicapra isabellina (Afz.). Isabelline Antelope. $H a b$. South Africa.

c. Female. Deposited Sept. 18, 1879.

481. Cephalophus rufilatus, Gray. Coquetoon Antelope. $H a b$. West Africa.

c. Female. Purchased, July 24, 1879.

490. Catoblepas gnu (Gm.). White-tailed Gnu.

$H a b$. South Africa.

g. Male. Purchased, July 17, 1879.

*492a. Capricornus crispus (Temm.). Japanese Goat-Antelope. Hab. Japan.

a. Male. Presented by H. Pryer, Esq., C.M.Z.S., April 12, 1879. See P.Z.S. 1879, p. 384. 
UNGULATA.

499. Ovis aries, Linn. Domestic Sheep.

y, z. Males. Presented by R. B. N. Walker, Esq., C.M.Z.S., Sept. 15, 1879.

$a^{1}, b^{1}$. Females. Presented by H. Sandbach, Esq., Nov. 22, 1879.

504. Camelopardatis giraffa, Gm. Giraffe.

Hab. Africa.

d'. Male. Purchased, Jan. 27, 1879. See P.Z.S. 1879, p. 108.

507. Cervulus reevesi, Ogilby. Reeves's Muntjac.

Hab. China.

n. Female. Born in the Menagerie, April 20, 1879.

o. Female. Born in the Menagerie, April 29, 1879.

p. Male. Born in the Menagerie, Nov. 28, 1879.

508. Elaphodus michianus (Swinhoe). Michie's Tufted Deer. $H a b$. China.

c. Male. Deposited May 27, 1879.

509. Cervus elaphus, Linn. Red Deer.

Hab. Europe.

n. Male. Hybrid between this species and $C$. barbarus Born in the Menagerie, Aug. 6, 1879.

515. Cervus sika, Temm. Japanese Deer.

Hab. Japan.

$x$. Male; $y, z$. Females. Presented by the Viscount Powerscourt, F.Z.S., March 6, 1879.

$a^{1}$. Male. Born in the Menagerie, July 7, 1879.

521. Cervus aristotelis, Cuv. Sambur Deer.

Hab. India.

$x$. Male. Born in the Menagerie, Feb. 27, 1879.

533. Cervus axis, Erxl. Axis Deer.

$H a b$. India.

$e^{1}$. Male. Born in the Menagerie, May 30, 1879. 
$f^{1}$. Female. Born in the Menagerie, July 1, 1879.

$g^{1}$. Male. Purchased, Nov. 6, 1879.

537. Hydropotes inermis, Swinhoe. Chinese Water-Deer. $H a b$. China.

e. Male. Received in exchange, April 3, 1879.

*538a. Capreolus pygargus (Pallas). White-rumped Roe-Deer. Hab. Eastern Asia.

$a, b$. Females. Received in exchange, April 3, 1879. See P.Z. S. 1879 , p. 384.

540. Cariacus mexicanus, H. Smith. Mexican Deer. Hab. Mexico.

v. Male. Hybrid between this species and C.virginianus . Born in the Menagerie, June 19, 1879.

*54.0a. Cariacus macrotis (Say). Mule Deer.

Hab. North America.

a. Male. Presented by Judge Caton, March 12, 1879. See P.Z. S. 1879 , p. 308.

b. Male. Presented by H. H. Carter, Esq., July 18, 1879.

c. Female. Presented by E. N. Carter, Esq., July 18, 1879. Wyoming Territory, U.S.A. See P.Z.S. 1879 , p. 664 .

543. C'ariacus rufus, F. Cuv. Red Brocket. Hab. Brazil.

e. Male; $f, g$. Female and young. Presented by W. H. Lacy, Esq., April 18, 1879.

\section{Order EDENTATA.}

552. Tatusia peba (Desm.). Peba Armadillo. Hab. South America.

c. Purchased, March 29, 1879. 
558. Dasypus vellerosus, Gray. Long-haired Armadillo. Hab. Mendoza.

b. Purchased, March 29, 1879.

561. Tamandua tetradactyla (Linn.). Tamandua Ant-eater. $H a b$. South America.

k. Purchased, May 3, 1879.

l. Purchased, June 14, 1879. From Pernambuco.

m. Purchased, Aug. 4, 1879.

n. Purchased, Dec. 31, 1879.

565. Orycteropus capensis (Gm.). Cape Ant-bear.

$H a b$. South Africa.

d. Purchased, March 25, 1879.

\section{Order MARSUPIALIA.}

567. Didelphys cancrivora, Gm. Crab-eating Opossum.

Hab. Tropical America.

e. Purchased, Sept. 3, 1879.

581. Phalangista vulpina (Shaw). Vulpine Phalanger.

$H a b$. Australia.

$v^{2}$. Female. Presented by A. Elder, Esq., May 14, 1879.

$w^{2}$. Presented by W. J. Lackey, Esq., Dec.. 21, 1879.

$x^{2}$. Bred in the Gardens, Dec. 26, 1879.

585. Belideus sciureus (Shaw). Squirrel-like Phalanger.

Hab. Australia.

c. Born in the Menagerie, Aug. 26, 1879.

590. Macropus rufus (Desm.). Red Kangaroo.

Hab. Australia.

t. Male. Born in the Menagerie, June 2, 1879.

593. Macropus giganteus, Shaw. Great Kangaroo.

$H a b$. New South Wales.

$i^{1}$. Male. Born in the Menagerie, June 2, 1879. 
594. Macropus melanops, Gould. Black-faced Kangaroo. $H a b$. South Australia.

h. Male. Purchased, April 19, 1879.

595. Petrogale xanthopus, Gray. Yellow-footed Rock-Kangaroo.

$\mathrm{Hab}$.

$e^{1}$. Born in the Menagerie, Feb. 12, 1879.

$f^{1}$. Male. Purchased, July 8, 1879.

597. Halmaturus bennettii, Waterh. Bennett's Wallaby. $H a b$. Tasmania.

$d^{1}$. Presented by W. E. Windus, Esq., Feb. 21, 1879.

$e^{1}$. Female. Born in the Menagerie, June 2, 1879.

605. Halmaturus brachyurus, Gould. Short-tailed Wallaby. Hab. Western Australia.

e. Presented by G. Bowen, Esq., Feb. 1, 1879.

609. Hypsiprymnus gaimardi (Desm.). Gaimard's Rat-Kangaroo.

Hab. Australia.

$x, y$. Presented by E. E. Harrold, Esq., March 12, 1879.

z. Born in the Menagerie, Nov. 17, 1879. 


\section{Class AVES.}

\section{Order PASSERES.}

1. Turdus musicus, Linn. Song-Thrush. Hab. British Islands.

f, g. Presented by F. J. Barry, Esq., Oct. 17, 1879.

5. Turdus migratorius, Linn. American Robin. Hab. North America.

$k$. Deposited, June 18, 1879.

8. Turdus albiventris, Spix. White-bellied Thrush. Hab. South America.

c. Purchased, Jan. 24, 1879. From Bahia.

*19a. Accentor collaris (Scopoli). Alpine Accentor. Hab. Europe.

a. Received in exchange, April 30, 1879. See P.Z.S. 1879, p. 384.

35. Cinclus aquaticus, Bechst. Water-Ouzel.

$H a b$. British Islands.

$t-x$. Presented by F. Swabey, Esq., April 28, 1879.

39. Motacilla yarrellii, Gould. Pied Wagtail.

$H a b$. British Islands.

s. Purchased, March 22, 1879.

$t, u$. Presented by A. F. Wiener, Esq., F.Z.S., June 18, 1879. $v-y$. Purchased, Oct. 28, 1879.

z. Purchased, Nov. 17, 1879.

44. Sitta casia, Meyer. Common Nuthatch.

Hab. British Islands.

h. Purchased, Feb. 25, 1879. 
53. Ampelis garrula (Linn.). Waxwing. Hab. Europe.

g. Presented by A. F. Wiener, Esq., F.Z.S., Sept 29, 1879.

*76a. Phyllornis hardwickii (Jard. et Selby). Blue-winged Green Bulbul.

\section{Hab. India.}

a. Presented by Mr. A. H. Jamrach, May 23, 1879.

84. Coreba cyanea (Linn.). Yellow-winged Sugar-bird. $H a b$. South America.

u. Purchased, Jan. 16, 1879.

89. Euphonia violacea (Linu.). Violet Tanager.

$H a b$. Brazil.

$t-v$. Purchased, June 14, 1879.

90. Euphonia crassirostris, Sclater. Thick-billed Tanager. Hab. Central America.

c. Purchased, Sept. 3, 1879.

95. Calliste fastuosa (Less.). Superb Tanager.

Hab. Pernambuco.

m. Purchased, Jan. 16, 1879.

n. Purchased, Sept. 3, 1879.

98. Calliste melanonota (Vieill.). Black-shouldered Tanager. $H a b$. Brazil.

b. Purchased, Sept. 3, 1879.

102. Tanagra palmarum (Max.). Palm-Tanager.

Hab. South America.

c. Purchased, Sept. 3, 1879.

*104a. Tachyphonus coronatus (Vieill.). Crowned Tanager. Hab. Brazil.

a. Male. Purchased, Sept. 3, 1879. 
*104b. Pyranga saira (Spix). Saira Tanager.

$H a b$. South America.

a. Male ; b. Female. Purchased, Jan. 24, 1879.

105. Ramploccelus brasilius (Linn.). Brazilian Tanager. Hab. Brazil.

d. Purchased, June 19, 1879.

116. Estrelda cinerea (Vieill.). Common Waxbill. $H a b$. West Africa.

m. Presented by J. C. Thorowgood, Esq., Oct. 13, 1879.

*128a. Pytelia wieneri, Finsch. Wiener's Finch. Hab. Africa.

a. Presented by A. F. Wiener, Esq., F. Z. S., June 18, 1879.

148. Vidua principalis (Linn.). Pin-tailed Whydah-bird. $H a b$. Africa.

c, d. Presented by Capt. J. H. Bowyer, Dec. 2, 1879.

172. Phonipara canora $(\mathrm{Gm}$.$) . Melodious Finch.$ Hab. Cuba.

d-i. Purchased, June 9, 1879.

177. Gubernatrix cristatella (Vieill.). Black-crested Cardinal. $H a b$. South America.

n, o. Purchased, July 23, 1879.

178. Diuca grisea (Less.). Diuca Finch. $H a b$. Chili.'

h. Purchased, Jan. 24, 1879. From Buenos Ayres.

184. Guiraca cyanea (Linn.). Brazilian Blue Grosbeak. Hab. Brazil.

e. Purchased, Jan. 24, 1879. From Mexico.

f. Purchased, Sept. 3, 1879. 
188. Cardinalis virginianus (Briss.). Cardinal Grosbeak. Hab. North America. $i^{1}, j^{1}$. Purchased, Feb. 18, 1879.

192. Coryphospingus pileatus (Max.). Pileated Finch. $H a b$. Brazil.

f. Male. Purchased, June 14, 1879.

195. Spermophila carulescens (Vieill.). Bluish Finch. Hab. Brazil. $j, k$. Purchased, June 14, 1879.

210. Ligurinus chloris (Linn.). Common Greenfinch. $H a b$. British Islands.

e,f. Presented by F. J. Barry, Esq., Oct. 17, 1879.

212. Fringilla coelebs, Linn. Common Chaffinch. $H a b$. British Islands. g-i. Presented by F. J. Barry, Esq., Oct. 17, 1879.

214. Fringilla montifringilla, Linn. Mountain-Finch. $\mathrm{Hab}$. British Islands. $k$, l. Purchased, March 8, 1879.

215. Carduelis elegans, Steph. Goldfinch. $H a b$. British Islands. $l, m$. Presented by H. A. Macpherson, Esq., Jan. 27, 1879. n, o. Presented by F. J. Barry, Esq., Oct. 17, 1879.

216. Chrysomitris spinus (Linn.). Common Siskin. $H a b$. British Islands. $l, m$. Purchased, Nov. 17, 1879.

220. Sycalis flaveola (Linn.). Saffron Finch. Hab. Brazil. o, p. Purchased, June 14, 1879. $q, r$. Bred in the Gardens, Oct. 7, 1879. 
229. Linota cannabina (Linn.). Common Linnct.

Hab. British Islands.

k. Presented by F. J. Barry, Esq., Oct. 17, 1879.

1, m. Purchased, Oct. 20, 1879.

231. Linota rufescens (Vieill.). Lesser Redpole.

Hab. British Islands.

$i, j$. Presented by Dr. Bree, F.Z.S., June 23, 1879.

$k, l$. Presented by F. J. Barry, Esq., Oct. 17, 1879

235. Pyrrhula europaa, Vieill. Common Bullfinch. Hab. Europe.

l. Male. Purchased, Feb. 21, 1879 (Russian variety).

237. Loxia curvirostra, Linn. Common Crossbill. Hab. British Islands.

m, n. Presented by H. A. Macpherson, Esq., July 31, 1879.

244. Emberiza miliaria, Linn. Common Bunting. Hab. British Islands.

$g, h$. Presented by J. Young, Esq., Sept. 27, 1879.

*244a. Emberiza citrinella (Linn.). Yellow Bunting.

$H a b$. British Islands.

a. Presented by F. J. Barry, Esq., Oct. 17, 1879.

$b, c$. Purchased, Oct. 20, 1879.

246. Emberiza schœeniclus, Linn. Reed-Bunting.

Hab. British Islands.

b. Purchased, March 22, 1879.

c. Purchased, March 31, 1879.

d. Purchased, Nov. 17, 1879.

$e, f$. Purchased, Dec. 29, 1879.

257. Zonotrichia pileata (Bodd.). Pileated Song-Sparrow.

Hab. North America.

e. Purchased, June 14, 1879. 
261. Alauda arvensis, Linn. Sky-Lark. Hab. British Islands.

b. Presented by F. Buckland, Esq., Aug. 25, 1879.

c. Presented by F. J. Barry, Esq., Oct. 17, 1879.

*272a. Eremophila chrysolama (Wagl.). White-bellied ShoreLark.

$H a b$. Cuba.

a-c. Purchased, June 9, 1879.

293. Pseudoleistes virescens (Vieill.). Dark Green Maizeeater.

Hab. South America.

b, c. Purchased, Jan. 24, 1879. From Buenos Ayres.

304. Sturnus vulgaris, Linn. Common Starling.

Hab. British Islands.

c, d. Presented by F. J. Barry, Esq., Oct. 17, 1879.

306. Acridotheres cristatellus (Linn.). Chinese Mynah. $H a b$. China.

h. Presented by A. F. Wiener, Esq., F.Z.S., Sept. 29, 1879.

311. Sturnia malabarica (Gm.). Malabar Mynah.

$H a b$. Hindostan.

$f, g$. Presented by A. F. Wiener, Esq., F.Z.S., Sept. 29, 1879.

316. Gracula religiosa, Linn. Small Hill-Mynah. $H a b$. Southern India.

i. Presented by J. W. Woodler, Esq., April 30, 1879.

317. Ptilonorhynchus violaceus, Vieill. Silky Bower-bird. Hab. New South Wales. $p-w$. Received in exchange, Nov. 11, 1879.

332. Pica rustica (Scopoli). Common Magpie. Hab. British Islands. m. Presented by J. L. Baldwin, Esq., June 11, 1879. 
339a. Garrulus lanceolatus, Vigors. Lanceolated Jay.

Hab. Himalayas.

$a, b$. Purchased, March 6, 1879.

$c$, d. Received in exchange, April 1, 1879. See P.Z.S. 1879, p. 384 .

340. Cyanocitta cristata (Linn.). Blue Jay.

$H a b$. North America.

l. Purchased, May 17, 1879.

341. Cyanocorax cyanopogon (Max.). Blue-bearded Jay. Hab. Para.

342. Cyanocorax pileatus (Temm.). Pileated Jay. Hab. La Plata.

e,f. Purchased, Jan. 24, 1879. Buenos Ayres.

g. Purchased, Aug. 7, 1879.

*344a. Cyanocorax cyanomelas (Vieill.). Black-headed Jay. $H a b$. South America.

Purchased, Dec. 11, 1879.

352. Dendrocitta sinensis (Lath.). Chinese Tree-pie. $H a b$. China.

d, e. Presented by Mr. Charles Rice, Oct. 14, 1879.

361. Gymnorhina leuconota, Gould. White-backed Piping Crow.

$H a b$. South Australia.

v. Presented by Mrs. Buchanan, Sept. 24, 1879 .

368. Chasmorhynchus nudicollis (Vieill.). Naked-throated Bellbird.

Hab. Brazil.

n, o. Purchased, Dec. 31, 1879.

370. Rupicola crocea, Vieill. Demeraran Cock-of-the-rock. Hab. Demerara.

h. Male. Presented by R. S. Fraser, Esq., Sept. 10, 1879. 


\section{Order CYPSELI.}

373. Podargus cuvieri, Vig. \& Horsf. Cuvier's Podargus. Hab. Australia.

h. Presented by R. S. C. Baber, Esq., April 25, 1879.

\section{Order PICI.}

375. Picus major, Linn. Greater Spotted Woodpecker. $H a b$. British Islands.

l. Presented by H. Laver, Esq., March 20, 1879.

\section{Order COCCYGES.}

385. Alcedo ispida, Linn. Kingfisher.

$H a b$. British Islands.

$d^{2}, e^{2}$. Presented by W. W. Cobb, Esq., June 5, 1879.

386. Dacelo gigantea (Lath.). Laughing Kingfisher. Hab. Australia.

71. Presented by F. Belcher, Esq., March 30, 1879. $m^{1}$. Presented by Mr. E. Hawkins, June 13, 1879.

399. Buceros subcylindricus, Sclater. Subcylindrical Hornbill. $H a b$. West Africa.

b. Received in exchange, May 8, 1879. See P.Z.S. 1879, p. 550.

401. Buceros atratus (Temm.). Black Hornbill.

Hab. West Africa.

d. Purchased, June 9, 1879.

e. Received in exchange, Aug. 4, 1879.

*413a. Corythaix porphyreolophus, Vigors. Purple - crested Touracou.

Hab. East Africa.

a. Presented by the Rev. J. A. Gould, F.Z.S., Feb. 4, 1879. See P.Z. S. 1879, p. 218. 


\section{Order PSI'TTACI.}

414. Cuculus canorus, Linn. Common Cuckoo.

Hab. British Islands.

s. Presented by Miss C. Bealey, Aug. 1, 1879.

t. Presented by Mr. J. Shapland, Aug. 18, 1879.

u. Presented by Mrs. Bolton, Sept. 15, 1879 .

v. Presented by Miss C. Bealey, Sept. 16, 1879.

423. Ramphastos carinatus, Sw. Sulphur-breasted Toucan. Hab. Mexico.

m. Purchased, Aug. 27, 1879.

427. Ramphastos ariel, Vig. Ariel Toucan.

Hab. Brazil.

g. Purchased, Jan. 24, 1879. From Bahia.

h. Purchased, May 17, 1879.

$i-l$. Purchased, Oct. 2, 1879.

*431a. Selenidera maculirostris (Licht.). Spotted-billed Toucanet.

$H a b$. Brazil.

a. Purchased, June 14, 1879. From Rio de Janeiro. See P.Z.S. 1879 , p. 663.

435. Cacatua moluccensis (Gm.). Rose-crested Cockatoo. Hab. Moluccas.

u. Presented by Miss Foster, Aug. 15, 1879.

436. Cacatua ophthalmica, Sclater. Blue-eyed Cockatoo. $H a b$. Solomon Islands.

e. Presented by Lt.-Col. Arbuthnot, July 4, 1876.

438. Cacalua galerita (Lath.). Greater Sulphur-crested Cockatoo.

Hab. Australia.

$k^{1}$. Deposited, April 3, 1879.

l1. Presented by J. W. Taylor, Esq., June 18, 1879. 
440. Cacatua sulphurea (Gm.). Lesser Sulphur-crested Cockatoo.

Hab. Moluccas.

o. Presented by Miss Langley, July 18, 1879.

447. Cacatua roseicapilla, Vicill. Roseate Cockatoo.

Hab. Australia.

n. Presented by Mr. Head, May 23, 1879.

448. Licmetis tenuirostris (Wagl.). Slender-billed Cockatoo. IIab. South Australia.

j. Presented by J. Wood, Esq., June 19, 1879.

452. Calyptorhynchus banksii (Lath.). Banksian Cockatoo. Hab. New South Wales.

c. Presented by Lady Ellesmere, Feb. 1, 1878.

*452a. Calyptorhynchus funereus (Shaw). Funereal Cockatoo. Hab. New South Wales.

a. Purchased, July 16, 1879. See P.Z.S. 1879, p. 663.

457. Lorius garrulus (Linn.). Chattering Lory. Hab. Moluccas.

f. Purchased, May 20, 1879.

g. Deposited, June 19, 1879.

474. Palcornis torquatus (Bodd.). Ring-necked Parrakeet. $H a b$. India.

$r^{1}$. Presented by F. S. Prince, Esq., May 14, 1879.

$s^{2}$. Presented by E. F. Carey, Esq., June 17, 1879.

$t^{1}$. Presented by Mrs. Watson, Aug. 22, 1879.

475. Palcoornis docilis (Vieill.). Rose-ringed Parrakeet. Hab. West Africa. i-l. Purchased, July 8, 1879.

479. Palcornis javanicus (Osb.). Javan Parrakeet. Hab. Java. $f, g$. Purchased, May 22, 1870. 
489. Polytelis melanura (Vig.). Black-tailed Parrakeet.

Hab. Australia.

f. Deposited, Aug: 30, 1879.

g. Purchased, Dec. 30, 1879.

491. Aprosmictus scapulatus (Kuhl). King Parrakeet.

$H a b$. New South Wales.

$c^{1}$. Male. Presented by Geo. Wood, Esq., Sept. 10, 1879.

$d^{i}$. Female. Presented by General Blake, Sept. 22, 1879.

500. Euphema pulchella (Shaw). Turquoisine Grass-Parrakeet.

- Hab. New South Wales.

l. Female. Presented by J. Fraser, Esq., Sept. 16, 1879.

$m$. Female. Presented by A. Battescombe, Esq., Nor. 26, 1879.

501. Euphema elegans, Gould. Elegant Grass-Parrakeet. $H a b$. South Australia.

$f-h$. Hybrids between a male of this species and a female Euphema pulchella. Bred in the Gardens, June 4, 1879.

$i$. Male ; $j$. Female. Purchased, Sept. 5, 1879.

505. Melopsittacus undulatus (Shaw). Undulated GrassParrakeet.

Hab. Australia.

$s^{2}$. Preseuted by Miss Balls, May 2, 1879.

$t^{2}, u^{2}$. Purchased, June 10, 1879 .

*๊10a. Nymphicus cornutus (Gm.) Horned Parrakeet.

$H a b$. New Caledonia.

a, b. Purchased, May 8, 1879. See P.Z.S. 1879, p. 550, pl. xurv.

512. Platycercus adelaida, Gould. Adelaide Broadtail. $\mathrm{Hab}$. South Australia.

b. Purchased, Aug. 23, 1879.

515. Platycercus eximius (Shaw). Rose-Hill Broadtail. $H a b$. New South Wales.

w. Presented by A. Stirling, Esq., Aug. 30, 1879. 
518. Platycercus flaviventris (Temm.). Yellow-bellied Broadtail.

$H a b$. Tasmania.

c, d. Purchased, July 17, 1879.

522. Cyanorhamphus nova-zealandia (Gm.). New-Zealand Parrakeet.

$H a b$. New Zealand.

e,f. Purchased, March 31, 1879.

528. Psephotus pulcherrimus, Gould. Beautiful Parrakeet. . Hab. Australia.

$l, m$. Purchased, June 24, 1879.

530. Pyrrhulopsis tabuensis (Lath.). Tabuan Parrakeet. Hab. Fiji Islands.

c. Deposited, March 17, 1879.

532. Pyrrhulopsis personata (Gray). Masked Parrakeet. $H a b$. Fiji Islands.

e. Purchased, April 10, 1879.

551. Ara macao (Linn.). Red-and-blue Macaw. $H a b$. Central America.

n. Presented by Chas. Fricke, Esq., July 23, 1879.

552. Ara chloroptera, Gray. Red-and-yellow Macaw. $\mathrm{Hab}$. South America.

$e^{1}$. Presented by Mrs. Kelly, April 1, 1879.

$f^{1}$. Presented by Miss C. Cattlin, June 5, 1879.

$g^{1}$. Purchased, June 12, 1879.

$h^{1}$. Deposited, July 18, 1879.

$i^{1}, j^{1}$. Presented by Chas. Fricke, Esq., July 23, 1879.

$k^{1}$. Deposited, Sept. 11, 1879.

553. Ara ararauna (Linn.). Blue-and-yellow Macaw.

$H a b$. South America.

$u$. Presented by F. G. J. Lillington, Esq., R.N., Jan. 24, 1879. 
v. Presented by Chas. Fricke, Esq., July 23, 1879.

w. Deposited, Sept. 11, 1879 .

*567a. Conurus auricapillus (Licht.). Golden-headed Conure. Hab. South America.

a, b. Purchased, May 22, 1879.

582. Bolborhynchus monachus (Bodd.). Grey-breasted Parrakeet.

Hab. Monte Video.

z. Presented by Miss Hilhouse, March 27, 1879.

*583a. Chrysotis bodini, Finsch. Bodinus's Amazon.

$H a b$. Venezuela.

a. Purchased, Dec. 18, 1879. See P.Z. S. 1880, p. 23.

*591a. Chrysotis erythrura (Kuhl). Red-tailed Amazon.

$H a b$. Brazil.

a. Purchased, Dec. 18, 1879. See P.Z.S. 1880, p. 23, pl. II.

595. Chrysotis dufresniana (Kuhl). Dufresne's Amazon.

Hab. South-east Brazil.

c. Presented by Mrs. T. Smith, Jan. 16, 1879.

d, e. Purchased, Oct. 20, 1879.

f. Received in exchange, Dec. 18, 1879.

*595a. Chrysotis cœligena. Blue-cheeked Amazon.

Hab. Guiana.

a. Purchased, Feb. 19, 1879. See P.Z. S. March 2, 1880.

597. Chrysotis guildingi, Vigors. Guilding's Amazon. $H a b$. St. Vincent, West Indies.

e. Presented by G. Dundas, Esq., April 26, 1879.

598. Chrysotis bouqueti, Bechst. Blue-faced Amazon. Hab. St. Lucia, West Indies.

d. Presented by Neville Holland, Esq., April 14, 1879. 
601. Chrysotis auripalliata (Less.). Golden-naped Amazon. $H a b$. Central America.

e. Presented by Mrs. H. A. Hopkins, Jan. 28, 1879.

606. Chrysotis ochrocephala (Gm.). Yellow-fronted Amazon. Hab. Guiana.

o. Presented by Mrs. T. Smith, Jan. 16, 1879.

$p, q$. Presented by Mrs. Kelly, A pril 1, 1879.

$r$ Presented by Neville Holland, Esq., April 14, 1879.

616. Brotogerys tiriacula (Bodd.). All-green Parrakeet. $H a b$. South America.

$i, j$. Presented by Dr. A. Stradling, Sẻpt. 5, 1879.

*620a. Brotogerys tuipara (Gm.). Golden-fronted Parrakeet. Hab. South America.

a. Received in exchange, Jan. 28, 1879.

622. Brotogerys tovi (Gm.). Tovi Parrakeet. Hab. Columbia.

b, c. Presented by Capt. H. King, May 2, 1879.

\section{Order STRIGES.}

623. Strix flammea, Linn. Common Barn-Owl. $H a b$. Europe, Asia, Africa, and America. $n^{1}, o^{1}$. Presented by R. A. Baldwin, Esq., July 8, 1879. $p^{1}$. Presented by Mr. H. Norris, Aug. 20, 1879. $q^{1}$. Presented by F. Bagnall, Esq., Nov. 4, 1879.

632. Syrnium aluco (Linn.). Wood-Owl. Hab. Europe.

$f^{1}$. Presented by Mrs. George Blagden, Feb. 13, 1879.

$g^{1}$. Presented by Capt. F. Lloyd, Aug. 6, 1879.

$h^{1}$. Presented by J. Smith, Esq., Nov. 25, 1879.

637. Bubo maximus, Fleming. Great Eagle-Ow]. Hab. Europe and North Asia.

t. Deposited, Jan. 7, 1879. 
642. Bubo maculosus (Vieill.). Spotted Eagle-Owl. Hab. Africa.

g. Purchased, Feb. 19, 1879.

648. Ketupa javanensis (Less.). Javan Fish-Owl. Hab. Java.

c. Purchased, May 19, 1879.

650. Scops yiu (Scop.). European Scops Owl.

Hab. South Europe.

b. Purchased, Oct. 11, 1879.

653. Pulsatrix torquata (Daud.). Downy Owl. Hab. South America.

g. Presented by Dr. A. Stradling, Nov. 15, 1879.

\section{Order ACCIPITRES.}

667. Buteo vulgaris, Leach. Common Buzzard. Hab. Europe.

z. Deposited, Aug. 16, 1879.

673. Buteo borealis (Gm.). Northern Buzzard. Hab. North America. $b, c$. Received in exchange, Jan. 28, 1879.

676. Archibuteo lagopus (Gm.). Rough-legged Buzzard. Hab. Europe.

o. Presented by Lord Hobart, Oct. 4, 1879. From Labrador.

689. Geranoaetus melanoleucus (Vieill.). Chilian Sea-Eagle.

Hab. South America.

n. Purchased, Jan. 23, 1879. From Buenos Ayres.

692. Thrasactus harpyia (Linn.). Harpy Eagle.

$H a b$. South America.

g. Purchased, Dec. 31, 1879. 
693. Helotarsus ecaudatus (Daud.). Bateleur Eagle. $H a b$. Africa.

j. Presented by Alex. Sinclair, Esq., Aug. 1, 1879.

696. Haliaetus leucoryphus (Pall.). Mace's Sea-Eagle. $H a b$. India.

b. Purchased, May 25, 1879.

c. Presented by Captain Butler, Nov. 14, 1879.

698. Haliaetus vocifer (Daud.). Vociferous Sea-Eagle. $\mathrm{Hab}$. Africa.

$i, j$. Presented by Dr. J. Kirk, C.M.Z.S., Aug. 6, 1879.

699. Aquila chrysaetos (Linn.). Golden Eagle.

Hab. Europe and North America.

$a^{1}$. Presented by the Earl of Dunmore, F.Z.S., June 4, 1879. From the Western Hebrides.

$b^{1}, c^{1}$. Presented by Mrs. A. H. Browne, July 22, 1879. From Scotland.

701. Aquila adalberti, Brehm. Spanish Imperial Eagle.

e. Presented by the Marquis de la Granja de San Saturnino, Aug. 2, 1879.

702. Aquila navioides, Cuv. Tawny Eagle.

Hab. Africa.

$f, g$. Presented by Dr. Holub, Sept. 18, 1879. From South Africa.

706. Nisaetus fasciatus Vieill.). Bonelli's Eagle. Hab. Europe.

i. Presented by Captain W. P. Forwood, Sept. 25, 1879.

707. Nisaetus pennatus (Gm.). Booted Eagle.

Hab. Europe.

d. Purchased, Oct. 5, 1879.

719. Melierax polyzonus, Rüpp. Many-zoned Hawk. $H a b$. East Africa.

f. Purchased, Feb. 20, 1879. 
723. Astur approximans, Vig. Allied Goshawk. Hab. Australia.

d. Purchased, June 17, 1879.

725. Falco peregrinus, Tunst. Peregrine Falcon. Hab. Europe.

$n-r$. Presented by Sir Matthew W. Ridley, Bart., Oct. 6, 1879. Scotland.

727. Falco barbarus, Linn. Barbary Falcon.

$H a b$. North Africa.

b. Deposited, Nov. 27, 1879.

728. Falco lanarius, Schleg. Lanner Falcon.

$H a b$. Eastern Europe.

i. Deposited, Aug. 4, 1879.

$j, k$. Deposited, Nov. 18, 1879.

742. Tinnunculus alaudarius (Gm.). Common Kestrel. $H a b$. British Islands.

$l^{1}-n^{1}$. Presented by the Rev. J. E. Campbell Colquhoun, Aug. 5,1879 .

$o^{1}$. Presented by Mr. R. Moon, Aug. 26, 1879.

745. Tinnunculus sparverius (Linn.). American Kestrel. Hab. America.

c. Purchased, July 14, 1879. From San Domingo.

747. Milus agyptius (Gm.). Egyptian Kite.

$H a b$. Africa.

$j, k$. Presented by A. Bells, Esq., June 19, 1879.

749. Milvus govinda, Sykes. Indian Kite. Hab. Eastern Asia.

c. Presented by Captain Murray, May 6, 1879. 
753. Herpetotheres cachinnans, Linn. Laughing Falcon. $H a b$. Brazil.

a. Purchased, June 30, 1879.

b. Purchased, Dec. 1, 1879.

759. Vultur monachus, Linn. Cinereous Vulture.

Hab. Europe.

f. Deposited, Nov. 13, 1879.

761. Vultur auricularis, Daud. Sociable Vulture. Hab. Africa.

g. Deposited, Sept. 18, 1879. From South Africa.

768. Gypaetus barbatus (Linn.). European Bearded Vulture. Hab. Europe.

c. Presented by Lord Lilford, F.Z.S., July 14, 1879.

d. Deposited, Sept. 3, 1879.

770. Serpentarius reptilivorus, Daud. Secretary Vulture. $H a b$. South Africa.

w. Presented by Dr. Holub, Sept. 18, 1879. Cradock, Cape Colony.

\section{Order STEGANOPODES.}

777. Pelecanus mitratus, Licht. Mitred Pelican. $H a b$. India.

c. Presented by Dr. Holub, Sept. 18, 1879. From Orange Free State, South Africa.

782. Fregata aquila (Linn.). Great Frigate-bird. $H a b$. America.

d. Purchased, Jan. 24, 1879. From Pernambuco.

783. Sula bassana (Linu.). Gannet.

Hab. British Islands.

o. Presented by E. F. Adams, Esq., Feb. 2, 1879. 


\section{Order HERODIONES.}

797. Ardea egretta, Gm. Great American Egret.

Hab. America.

g. Purchased, May 3, 1879.

h. Purchased, June 14, 1879.

*800a. Ardea russata, Wagler. Buff-backed Egret.

Hab. Europe.

a. Presented by Captain Burke, S.S. 'Arcot,' July 12, 1879.

*805a. Ardetta involucris (Vieill.). Variegated Bittern.

Hab. South America.

$a, b$. Purchased, November 6, 1879 .

806. Nycticorax griseus (Linn.). Common Night-Heron.

Hab. Europe.

g. Purchased, Nov. 6, 1879.

810. Nycticorax violaceus (Linn.). Violaceous Night-Heron. Hab. South America.

$h, i$. Purchased, Jan. 24, 1879. From Bahia.

811. Cancroma cochlearia, Linn. Boatbill.

Hab. South America.

g, h. Purchased, Nov. 6, 1879.

815. Ciconia nigra, Bechst. Black Stork.

Hab. Europe.

$h, i$. Purchased, Aug. 8, 1879.

j. Presented by Prof. J. Reinhardt, F.M.Z.S., Aug. 13, 1879.

816. Ciconia alba, Linn. White Stork.

Hab. Europe.

$q-t$. Purchased, June 27, 1879.

u-v. Purchased, July 1, 1879. 
830. Tantalus loculator, Linn. American Tantalus. Hab. America.

c. Received in exchange, Jan. 28, 1879.

831. Platalea leucorodia, Linn. Common Spoonbill. Hab. Europe.

$f$-i. Purchased, Aug. 22, 1879.

840. Theristicus caudatus (Bodd.). Black-faced Ibis. $H a b$. South America.

g. Presented by C. H. Whaley, Esq., Aug 8, 1879.

842. Eudocimus ruber (Linn.). Scarlet Ibis. Hab. Para.

$d^{3}$. Purchased, May 2, 1879.

843. Eudocimus albus (Linn.). White Ibis.

Hab. South America.

g. Purchased, May 2, 1879.

846. Phonicopterus ignipalliatus, Geoffr. et d'Orb. SouthAmerican Flamingo.

Hab. South America.

c. Purchased, May 2, 1879.

\section{Order ANSERES.}

847. Chauna chavaria (Linn.). Crested Screamer. Hab. Buenos Ayres.

e. Purchased, June 14, 1879. Rio de Janeiro.

853. Chenalopex agyptiaca (Linn.). Egyptian Goose. Hab. Africa.

$u^{1}-w^{1}$. Bred in the Gardens, June 4, 1879. 
854. Chenalopex jubata (Spix). Orinoco Goose.

Hab. South America.

$k, l$. Purchased, June 9, 1879.

856. Anser ferus, Linn. Wild or Grey-lag Goose.

Hab. British Islands.

e. Presented by R. J. Balston, Esq., F.Z.S., $\Lambda$ pril 4, 1879.

858. Anser segetum, Linn. Bean-Goose.

Hab. Europe.

d. Presented by R. J. Balston, Esq., F.Z.S., April 4, 1879.

863. Bernicla leucopsis, Bechst. Bernicle Goose.

Hab. Europe.

g, h. Presented by R. J. Balston, Esq., F.Z.S., April 4, 1879.

864. Bernicla canadensis (Linn.). Canada Goose.

Hab. British Islands.

Zn. Presented by W. Bonorton, Esq., Jan. 30, 1879.

o-r. Presented by R. J. Balston, Esq., F.Z.S., April 4, 1879.

867. Bernicla brenta, Steph. Brent Goose.

Hab. Europe.

w. Presented by H. A. Dombrain, Esq., March 11, 1879.

$x, y$. Presented by R. J. Balston, Esq., F.Z.S., April 4, 1879.

$z$, $a^{1}$. Purchased, Dec. 11, 1879.

868. Bernicla jubata (Lath.). Maned Goose.

Hab. Australia.

n. Male; $0, p$. Females. Purchased, June 5, 1879.

870. Bernicla magellanica (Gm.). Upland Goose.

$H a b$. Falkland Islands.

j. Male. Purchased, April 18, 1879 . Patagonia (var. $B$. dispar).

k. Female. Purchased, Sept. 5, 1879. 
871. Bernicla poliocephala, Sclater. Ashy-headed Goose. Hab. South America.

n. Purchased, Sept. 5, 1879.

879. Cygnus nigricollis (Gm.). Black-necked Swan.

Hab. Antarctic America.

w. Bred in the Gardens, May 23, 1879.

880. Cygnus atratus, Lath. Black Swan.

Hab. Australia.

$b^{1}$. Male ; $c^{1}$. Female. Received in exchange, May 19, 1879. $d^{2}-g^{1}$. Purchased, July 12, 1879.

882. Dendrocygna viduata (Linn.). White-faced Tree-Duck. $H a b$. Brazil.

$x$. Purchased, Jan. 24, 1879. From Rio de Janeiro. $y, z$. Purchased, July 29, 1879. From Rio Magdalena.

883. Dendrocygna autumnalis (Linn.). Red-billed Tree-Duck. $H a b$. America.

$d^{1}-f^{1}$. Purchased, May 2, 1879.

$g^{1}-i^{1}$. Purchased, June 30, 1879.

$k^{1}$. Purchased, July 29, 1879. Rio Magdalena.

$l^{1}$. Purchased, Sept. 23, 1879.

891. Tadorna vulpanser, Flem. Common Sheldrake. Hab. Europe. $r, s$. Males; $t$, $u$. Females. Purchased, May 25, 1879.

892. Tadorna rutila (Pall.). Ruddy Sheldrake. Hab. Europe. $i^{2}$. Male; $j^{2}$. Female. Presented by R. J. Balston, Esq.á F.Z.S., April 4, 1879.

896. Anas boschas, Linn. Common Wild Duck. Hab. Europe. r-t. Presented by R. J: Balston, Esq., F.Z.S., April 4, 1879. 
898. Anas superciliosa, Gm. Australian Wild Duck.

$H a b$. Australia.

$e^{3}-g^{3}$. Purchased, Feb. 17, 1879.

$h^{3}-9^{3}$. Purchased, April 4, 1879.

$k^{3}-n^{3}$. Presented by Messrs. A. H. Jamrach and C. Rice, June 17, 1879.

$o^{3}-q^{3}$. Bred in the Gardens, June 23, 1879.

$r^{3}-t^{3}$. Bred in the Gardens, July 11, 1879.

$u^{s}$. Bred in the Gardens, Aug. 7, 1879.

899. Anas pœcilorhyncha, Peun. Spotted-billed Duck.

$H a b$. India.

$d^{2}-f^{2}$. Bred in the Gardens, June 23, 1879.

900. Anas xanthorhyncha, Forst. Yellow-billed Duck.

$H a b$. South Africa.

s. Bred in the Gardens, July 11, 1879.

901. Anas castanea, Eyton. Chestnut-breasted Duck.

$H a b$. Australia.

९-v. Purchased, Aug. 22, 1879.

902. Anas strepera, Linn. Gadwall Duck.

Hab. Europe.

f. Male; g. Female. Presented by R. J. Balston, Esq., F.Z.S., April 4, 1879.

904. Querquedula crecca (Linn.). Common Teal.

$H a b$. Europe.

$d^{1}$. Male ; $e^{1}$. Female. Presented by R. J. Balston, Esq., F.Z.S., April 4, 1879.

$f^{1}-k^{1}$. Males; $l^{1}-q^{1}$. Females. Purchased, April 4, 1879.

905. Querquedula brasiliensis (Gm.). Brazilian Teal.

Hab. South America.

$u-a^{1}$. Bred in the Gardens, Aug. 7, 1879.

508. Querquedula creccoides (King). Chilian Teal.

Hab. Antarctic America.

i. Presented by Mr. W. Petty, Sept. 5, 1879. 
909. Querquedula circia (Linn.). Garganey Teal. $H a b$. Europe.

q. Male; r. Female. Presented by R. J. Balston, Esq., F.Z.S., April 4, 1879.

$s-x$. Males ; $y-d^{2}$. Females. Purchased, May 7, 1879.

910. Dafila acuta (Linn.). Common Pintail. $H a b$. Europe.

v. Male ; $w$. Female. Presented by R. J. Balston, Esq., F.Z.S., April 4, 1879.

$x$. Male; $y$. Female. Purchased, May 7, 1879.

911. Dafila spinicauda (Vieill.). Chilian Pintail.

$H a b$. Antarctic America.

$j^{3}$. Male; $k^{3}$. Female. Presented by R. J. Balston, Esq., F.Z.S., April 4, 1879.

$l^{3}-n^{3}$. Bred in the Gardens, June 23, 1879.

$0^{3}-q^{3}$. Bred in the Gardens, Aug. 7, 1879.

$r^{3}$. Presented by Mr. W. Petty, Sept. 5, 1879.

914. Mareca penelope (Linn.). Wigeon.

Hab. Europe.

$k^{1}$. Presented by F. Cresswell, Esq., Jan. 23, 1879.

$l^{1}$. Male; $m^{1}$. Female. Presented by R. J. Balston, Esq., F.Z.S., April 4, 1879.

$n^{1}$. Male; $o^{1}$. Female. Purchased, May 7, 1879.

$p^{1}-u^{1}$. Males; $v^{1}-a^{2}$. Females. Purchased, May 24, 1879.

915. Mareca chiloensis (King). Chiloe Wigeon.

Hab. Chili.

$y^{2}, z^{2}$. Males; $a^{3}, b^{3}$. Females. Presented by R. J. Balston, Esq., F.Z.S., A pril 4, 1879.

$c^{3}, d^{3}$. Bred in the Gardens, June 15, 1879.

916. Spatula clypeata (Linn.). Shoveller.

Hab. Europe."

$y, z$. Males; $a^{1}, b^{1}$. Females. Purchased, May 7, 1879.

917. Aix sponsa (Linn.). Summer-Duck.

Hab. North America.

$m^{4}$. Male. Presented by R. J. Balston, Esq., F.Z.S., April 4, 1879. 
918. Aix galericulata (Iinn.). Mandarin Duck.

Hab. China.

$w^{2}$. Male; $x^{2}$. Temale. Presented by R. J. Balston, Esq., F.Z.S., April 4, 1879.

919. Metopiana peposaca (Vieill.). Rosy-billed Duck.

Hab. South America.

$z-e^{1}$. Bred in the Gardens, July 11, 1879.

920. Fuligula cristata (Leach). Tufted Duck.

Hab. Europe.

$r-t$. Males; $u-w$. Females. Purchased, May 7, 1879.

923. Fuligula ferina (Linn.). Red-headed Pochard.

Hab. Europe.

$e-i$. Males; $k-p$. Females. Purchased, May 7, 1879.

\section{Order COLUMB王.}

957. Columba guinea, Linn. Triangular-spotted Pigeon. $H a b$. West Africa.

i. Presented by Col. F. C. Hassard, C.B., Jan. 12, 1879.

961. Columba speciosa, Gm. Specious Pigeon.

Hab. South America.

$h-k$. Received in exchange, Aug. 4, 1879.

974. Geopelia striata (Linn.). Barred Dove. $H a b$. India.

o, p. Presented by Capt. H. Braddick, Feb. 17, 1879 .

987. Turtur chinensis (Scop.). Chinese Turtledove. $H a b$. India.

i. Presented by Capt. H. Braddick, Feb. 17, 1879. 
995. Peristera geoffroii (Temm.). Geoffroy's Dove. Hab. Brazil.

w. Bred in the Gardens, Feb. 3, 1879.

$x, y$. Bred in the Gardens, May 13, 1879.

$z$. Bred in the Gardens, May 26, 1879.

$a^{1}, b^{1}$. Bred in the Gardens, June 11, 1879.

$c^{1}, d^{1}$. Bred in the Gardens, June 30, 1879.

$e^{1}, f^{1}$. Bred in the Gardens, July 24, 1879.

$g^{1}, h^{1}$. Bred in the Gardens, Aug. 14, 1879.

$i^{1}$. Bred in the Gardens, Dec. 22, 1879.

997. Zenaida martinicana (Bp.). Martinican Dove. Hab. West Indies.

g, h. Presented by Capt. H. King, Sept. 8, 1879.

1002. Chamapelia passerina (Linn.). Passerine Ground-Dove. Hab. America.

g, h. Presented by Capt. H. King, May 2, 1879.

1013. Ocyphaps lophotes (Temm.). Crested Pigeon. Hab. Australia.

$u^{1}$. Presented by the Rev. A. H. Glennie, June 23, 1879.

$v^{1}, w^{1}$. Bred in the Gardens, Aug. 14, 1879.

*1020a. Phlogoenas stairi (G. R. Gray). Stair's Ground-Dove. Hab. Fiji Islands.

a. Purchased, May 16, 1879. (Deposited, March 17, 1879.)

1021. Caloenas nicobarica (Linn.). Nicobar Pigeon. Hab. Indian archipelago.

s. Purchased, May 22, 1879.

1023. Goura coronata (Linn.). Common Crowned Pigeon. $H a b$. New Guinea.

o. Purchased, June 9, 1879.

$p$-s. Purchased, July 17, 1879. 
1024. Goura victoria, Fraser. Victoria Crowned Pigeon. IIab. Island of Jobie.

f. Purchased, May 25, 1879.

\section{Order GALLIN Æ.}

1067. Odontophorus guianensis (Gm.). Guiana Partridge. Hab. Guiana.

j. Presented by E. L. Marshall, Esq., May 14, 1879.

1071. Eupsychortyx cristatus (Linn.). Crested Colin. Hab. Mexico.

c. Male; d. Female. Purchased, Oct. 14, 1879.

1073. Callipepla californica (Shaw). Californian Quail. $H a b$. California.

k, l. Presented by Mr. W. Turquand, Jan. 1, 1879.

1081. Ammoperdix heyi (Temm.). Hey's Partridge. $H a b$. Arabia.

m. Presented by Capt. Burke, July 12, 1879 .

1083. Lophophorus impeyanus (Lath.). Himalayan Monaul. Hab. Himalayan Mountains.

$c^{2}$. Female. Received in exchange, Feb. 25, 1879.

1092. Phasianus wallichii, Hardw. Cheer Pheasant. $H a b$. North India.

$v^{1}$. Male; $w^{1}$. Female. Received in exchange, Feb. 25, 1879.

1093. Thaumalea picta (Linn.). Gold Pheasant. Hab. China.

$j^{2}$. Male. Presented by J. E. Liardet, Esq., July 12, 1879. 
1094. Thaumalea amherstia (Leadb.). Amherst's Pheasant. $H a b$. Szechuen, China.

$u^{3}-x^{3}$. Bred in the Gardens, June 5, 1879 .

$y^{3}$. Bred in the Gardens, Aug. 7, 1879.

1096. Pucrasia darwini, Swinh. Darwin's Pucras.

Hab. China.

v. Male. Purchased, July 8, 1879.

1098. Euplocamus pralatus (Bonap.). Siamese Pheasant. Hab. Siam.

$t, u$. Females. Purchased, July 8, 1879.

1103. Euplocamus nyctemerus (Linn.). Silver Pheasant. Hab. China.

$a^{1}$. Male; $b^{1}$. Female. Presented by W. Soper, Esq., April 1, 1879.

$c^{2}$. Male. Presented by Mrs. E. J. Beagle,'A pril 29, 1879.

$d^{1}$. Male. Presented by Mr. R. Moon, Oct. 27, 1879.

1111. Gallus varius, Shaw. Fork-tailed Jungle-fowl. Hab. Java.

o-q. Bred in the Gardens, Aug. 7, 1879.

1117. Pavo cristatus, Linn. Common Peafowl. $H a b$. India.

$h^{1}$. Male. Presented by J. B. Hopkinson, Esq., March 28, 1879.

1118. Pavo nigripennis, Sclater. Black-winged Peafowl. Hab. Cochin China.

$m$. Presented by the Hon. A. S. G. Canning, F.Z.S., Feb. 1, 1879.

n. Female. Presented by the Hon. A. S. G. Canning, F.Z.S., July 8, 1879.

1119. Pavo spicifer, Horsf. Javan Peafowl. $H a b$. Burmah and Java.

$o, p$. Purchased, May 24, 1879.

$q-s$. Deposited, June 5, 1879.

$t$. Male. Received in exchange, Oct. 10, 1879. 
1120. Polyplectron chinquis, Temm. Peacock Pheasant. Hab. British Burmah. $y^{1}-a^{2}$. Males: $b^{2}, c^{2}$. Females. Purchased, May 10, 1879. d. Bred in the Gardens, July 23, 1879.

1122. Argus giganteus, Temm. Argus Pheasant. Hab. Malacca.

r. Male. Received in exehange, May 26, 1879.

1124. Meleagris gallo-pavo, Linn. North-American Turkey. $H a b$. North America.

$x^{1}$. Male; $y^{1}, z^{1}$. Females. Presented by R. Wynne Roberts, Esq., Aug. 5, 1879.

1126. Numida ptilorhyncha, Licht. Abyssinian Guinea-fowl. Hab. Abyssinia.

g-i. Purchased, May 22, 1879.

1127. Numida rendalli, Ogilby. Rendall's Guinea-fowl. $H a b$. West Africa. $c, d$. Purchased, Oct. 6, 1879.

1129. Numida mitrata, Pallas. Mitred Guinea-fowl. $H a b$. Madagascar. e-g. Deposited, Aug. 6, 1879, and purchased Oct. 2, 1879.

1130. Numida cristata, Pall. Crested Guinea-fowl. Hab. West Africa.

w. Presented by Vice-Admiral John Corbett, C.B., Oct. 3, 1879.

*1130a. Numida ellioti, Bartlett. Elliot's Guinea-fowl.

$H a b$. East Africa.

$a, b$. Deposited, July 12, 1879.

c $f$. Deposited, Aug. 6, 1879 . One died Aug. 6, on Aug. 9 , one Aug. 18 ; survivor purchased, Oct. 2, 1879. See P.Z. S. 1879 , p. 718.

$g, h$. Deposited, Dec. $7,1879$. 
1131. Numida vulturina, Hardw. Vulturine Guinea-fowl. Hab. Eastern Africa.

$x-a^{1}$. Deposited, July 12,1879 .

$b^{1}$. Deposited, Aug. 6, 1879, and purchased, Oct. 2, 1879.

$c^{1}$. Deposited, Aug. 7, 1879, died Aug. 25, 1879.

$d^{1}-f^{1}$. Presented by Vice-Admiral John Corbett, C.B., Oct. 3, 1879.

1132. Crax globicera, Linn. Globose Curassow. Hab. Central America.

y. Presented by the Rev. Ralph Cooper, March 28, 1879,

z. Purchased, June 12, 1879.

$a^{1}-c^{1}$. Presented by Major F. Hime, July 14, 1879.

1135. Crax sclateri, G. R. Gray. Sclater's Curassow. Hab. South America.

k. Purchased, May 17, 18 79.

\section{Order FULICARIÆ.}

1170. Rallus aquaticus, Linn. Water-Rail.

Hab. British Islands.

i. Purchased, Nov. 11, 1879.

*1172a. Rallus maculatus (Bodd.). White-spotted Rail. Hab. South America.

$a, b$. Purchased, Nov. 6, 1879.

*1172b. Rallus rhythirhynchus (Vieill.). Sooty Rail. $H a b$. South America.

a. Purchased, Nov. 6, 1879.

*1173a. Rallina pœciloptera (Hartl.). Bar-winged Rail. Hab. Fiji Islands.

a. Purchased, Jan. 6, 1879. See P.Z.S. 1879, p. 108. 
1186. Porphyrio madagascariensis (Gm.). Madagascar Porphyrio.

Hab. Africa.

g-i. Presented by A. Bells, Esq., Juno 19, 1879.

1188. Porphyrio alleni, Thomps. Allen's Porphyrio.

Hab. West Africa.

g. Presented by W. B. Brown, Esq., July 31, 1879. Captured at sea off Sierra Leone, $5^{\circ} \mathrm{N}$., $14^{\circ} \mathrm{W}$.

1189. Porphyrio martinicus (Linn.). Martinique Waterhen.

Hab. South America.

7. Purchased, June 17, 1879.

\section{Order ALECTORIDES.}

1201. Otis tarda, Linn. Great Bustard.

Hab. Europe.

k. Male; 7. Female. Presented by J. C. Forster, Esq., Sept. 2, 1879. From Andalusia.

m. Female. Presented by Geo. G. Sandeman, Esq., Oct. $16,1879$.

1208. Houbara undulata (Jacq.). Houbara Bustard. Hab. North Africa.

j. Purchased, Dec. 16, 1879.

1212. Psophia crepitans, Linn. Common Trumpeter. Hab. Demerara.

m. Presented by Chas. Fricke, Esq., July 23, 1879.

1213. Psophia viridis, Spix. Green-winged Trumpeter. Hab. Brazil.

f. Purchased, June 9, 1879.

1215. Cariama cristata (Linn.). Brazilian Cariama. Hab. South-east Brazil.

i. Purchased, June 14, 1879. Rio de Janeiro. 
1228. Tetrapteryx paradisea, Licht. Stanley Crane. $H a b$. South Africa.

n. Received in exchange, July 22, 1879.

o. Deposited, Sept. 18, 1879.

1229. Balearica pavonina (Linn.). Balearic Crowned Crane. Hab. West Africa.

e,f. Purchased, July 8, 1879.

1230. Balearica regulorum, Bennett. Cape Crowned Crane. $H a b$. South Africa.

h, i. Deposited, Sept. 18, 1879.

1231. Eurypyga helias (Pall.). Sun-Bittern.

Hab. South America.

$j^{1}$. Purchased, June 14, 1879.

$k^{1}$. Deposited, Oct. 14, 1879.

\section{Order LIMICOL屟.}

1239. Vanellus cayennensis (Gm.). Cayenne Lapwing. $H a b$. South America. $l, m$. Purchased, Aug. 27, 1879.

1243. Squatarola helvetica (Linn.). Grey Plover. Hab. British Islands. $e-h$. Presented by F. Cresswell, Esg., Jan. 23, 1879.

1246. Agialitis hiaticula (Linn.). Ringed Plover. $H a b$. British Islands.

c. Presented by E. A. T. Elliott, Esq., Sept. 19, 1879.

1249. Strepsilas interpres (Linn.). Turnstone. Hab. Europe.

e. Presented by E. A. T. El:iot, Esq., Sept. 19, 1879. 
1250. Chionis alba, Forst. Yellow-billed Sheathbill. Hab. Antarctic America. $e, f$. Purchased, Feb. 10, 1879.

1252. Numenius arquata, Linn. Common Curlew. Hab. Europe, Asia, Africa. j. Deposited, Nov. 20, 1879. k. Purchased, Dec. 1, 1879.

1253. Numenius phaopus (Linn.). Whimbrel. $H a b$. Europe.

c. Received in exchange, July 2, 1879.

1256. Limosa lapponica (Linn.). Bar-tailed Godwit. $H a b$. Europe.

d. Purchased, Dec. 1, 1879.

1257. Limosa melanura, Leisler. Black-tailed Godwit. Hab. Europe. $c, c$. Purchased, May 19, 1879. $e, f$. Purchased, June 24, 1879.

1258. Scolopax rusticula, Linn. Woodcock. $H a b$. Europe.

c. Presented by Mr. J. Pollard, Nov. 13, 1879.

1263. Tringa cinclus, Linn. Dunlin.

Hab. Europe.

$m^{1}$. Presented by F. Cresswell, Esq., Jan. 23, 1879.

$n^{1}, o^{1}$. Presented by A. E. T. Eilliot, Esq., Sept. 19, 1879.

1264. Tringa canutus, Linn. Knot.

Hab. Europe.

$z-b^{1}$. Presented by F. Cresswell, Esq., Jan. 23, 1879.

1265. Himantopus nigricollis, Vieill. Black-necked Stilt. Hab. South America. d. Purchased, March 16, 1879. e-g. Purchased, Aug. 27, 1879. 


\section{Order GAVIÆ.}

1270. Stercorarius pomatorhinus (Temm.). Pomatorhine Skua. Hab. Europe.

b. Presented by F. L. Smith, Esq., Nov. 13, 1879.

c, d. Purchased, Dec. 5, 1879.

1274. Larus argentatus, Gm. Herring-Gull. Hab. Europe.

$y^{1}$. Presented by Miss J. Windsor, May 10, 1879.

$z^{1}$. Presented by C. H. C. de Loecker, Esq., May 15, 1879.

1275. Larus cachinnans, Pallas. Yellow-legged Herring-Gull. Hab. North Africa.

$i, j$. Bred in the Gardens, May 17, 1879 .

$k, l$. Bred in the Gardens, June 9, 1879.

1276. Larus fuscus, Linn. Lesser Black-backed Gull. $H a b$. British Islands.

r. Presented by the Rer. F. H. Addams, F.Z.S., Oct. 23, 1879.

1277. Larus canus, Linn. Common Gull.

Hab. British Isles.

h, i. Presented by F. Cresswell, Esq., Jan. 23, 1879.

j. Presented by H. W. Preston, Esq., Feb. 14, 1879.

1281. Larus ridibundus, Linn. Black-headed Gull. Hab. Europe.

$v, w$. Presented by Harry W. Preston, Esq., Feb. 14, 1879.

1283. Larus nova-hollandia, Steph. Jameson's Gull.

5a. Hab. Australia.

$p, q$. Bred in the Gardens, June 19, 1879.

1289. Anous stolidus (Linn.). Noddy Tern. $H a b$. West Indies.

b. Presented by M. H. Smyth, Esq., R.N., Jan. 17, 1879. 


\section{Order PYGOPODES.}

1292. Podiceps minor, Lath. Little Grebe.

Hab. British Islands.

q. Presented by A. F. Buxton, Esq., Nov. 17, 1879.

*1294a. Colymbus septentrionalis, Lath. Red-throated Diver. Hab. Europe.

a. Presented by J. S. Thompson, Esq., April 8, 1879.

b. Deposited, Nov. 20, 1879.

\section{Order IMPENNES.}

1298. Aptenodytes pennanti, Gray. King Penguin. Hab. Falkland Islands.

e. Purchased, Nov. 14, 1879. See P.Z. S. 1879, p. 763. From Staten Island, Cape Horn.

1300. Spheniscus demersus (Linn.). Black-footed Penguin. Hab. South Africa.

$i, j$. Purchased, Aug. 14, 1879.

\section{Order CRYPTURI.}

1307. Crypturus noctivagus, Max. Banded Tinam Hab. Brazil.

k. Received in exchange, Aug. 4, 1879.

\section{Order STRUTHIONES.}

1314. Rhea americana, Vieill. Common Rhea. $H a b$. South America.

$a^{1}$. Presented by Major Venables, Feb. 7, 1879 . 


\section{Class REPTILIA. \\ Order TESTUDINATA.}

5. Testudo pardalis, Bell. Leopard Tortoise. $H a b$. South Africa.

g. Purchased, May 1, 1879.

7. Testudo radiata, Shaw. Radiated Tortoise. Hab. Madagascar.

l. Purchased, Aug. 27, 1879.

12. Testudo carbonaria, Spix. Black Tortoise. Hab. South America. $u, v$. Presented by Capt. H. King, Sept. 8, 1879.

15. Testudo elephantina, Günther. Elephantine Tortoise. $H a b$. Aldabra Island.

b. Deposited, Dec. 4, 1879.

36. Clemmys punctularia (Daudin). Rough Terrapin. $H a b$. Trinidad.

h. Received in exchange, Jan. 28, 1879.

i. Presented by Surgeon-Major C. J. Weir, May 16, 1879.

37. Clemmys ornata (Bell). Adorned Terrapin.

$H a b$. Central America.

m. Presented by Surgeon-Major C. J. Weir, May 16, 1879.

\section{Order CROCODILIA.}

82. Alligator mississippiensis (Daudin). Alligator. Hab. Mississippi.

$x, y$. Presented by Lord Francis Conyngham, M.P., F.Z.S., April 3, 1879.

z. Presented by Capt. J. H. Mortimer, Oct. 25, 1879.

$a^{1}$. Presented by W. G. Marshall, Esq., Dec. 6, 1879. 
83. Alligator latirostris (Daudin). Broad-snouted Cayman. $H a b$. South America.

c. Presented by Dr. A. Stradling, April 2, 1879.

\section{Order SAURIA.}

94. Sphenodon punctatus (Gray). Tuatera Lizard. Hab. New Zealand.

$c, d$. Purchased, June 24, 1879. From the island of " Karewa, Bay of Plenty, N. Z." See P. Z.S. 1879, p. 663.

95. Chamaleon vulgaris, Daudin. Common Chameleon. $H a b$. North Africa.

$y^{3}$. Presented by G. A. Dodd, Esq., May 6, 1879.

$z^{3}, a^{4}$. Presented by A. Ely, Esq., Sept.5, 1879. Cyprus.

$b^{4}, c^{4}$. Presented by Capt. Burke, Dec. 5, 1879.

*98a. Amphisbana darwini, Dum.et Bibr.Darwin's’Amphisbæna. Hab. South America.

a. Purchased, Jan. 16, 1879. From Monte Video. See P.Z.S. 1879 , p. 146.

*101a. Phyllodactylus europaus, Gené. European Gecko. Hab. Europe.

a-e. Presented by Prof.H.H. Giglioli, C.M.Z.S., March 25, 1879.

106. Uromastix microlepis, Blanford. Small-scaled Mastigure. Hab. Busreh, Persian Gulf. $f-k$. Presented by Capt. Burke, S.S. ‘Arcot,' July 12, 1879.

112. Iguana tuberculata, Laur. Tuberculated Iguana. $H a b$. West Indies.

v. Presented by Dr. A. Stradling, Jan. 16, 1879.

$w$. Presented by Dr. J. F. Chittenden, C.M.Z.S., April 2, 1879.

$x$. Presented by Dr. A. Stradling, June 14, 1879. 
*117a. Taraguira smithi, Gray. Taraguira Lizard.

Hab. Brazil.

a. Presented by Dr. A. Stradling, June 14, 1879. From Bahia.

117b. Crotaphytus wislizenii, Baird et Gir. Wizlezen's Lizard. Hab. New Mexico.

a. Presented by Lt.-Col. Ralph Vivian, F.Z.S., Feb. 18, 1879, See P.Z. S. 1879, p. 218.

118. Phrynosoma cornutum (Harlan). Horned Lizard. $H a b$. Texas.

$d^{1}, e^{1}$. Presented by E. Loder, Esq., F.Z.S., May 23, 1879.

$f^{1}-h^{1}$. Presented by E. E. Sabel, Esq., F.R.G.S., Aug. 31, 1879.

125. Psammosaurus caspicus (Eichw.). Caspian Ouaran.

Hab. Persia.

c. Presented by Commander J. Pratt, June 15, 1879.

126. Teius teguexin (Iinn.). Common Teguexin.

Hab. South America.

$m$. Purchased, May 4, 1879.

129. Lacerta viridis, Linn. Green Lizard.

$H a b$. Island of Jersey.

$a^{3}$. Presented by the Rer. S. R. Wilkinson, F.Z.S., Oct. 3, 1879.

149. Anguis fragilis, Linn. Slowworm.

$\mathrm{Hab}$. British Isles.

m. Presented by W. A. H. Bernhard Smith, Esq., Dec. 7, .1879.

*152a. Acontias meleagris, Cuv. Spotted Slowworm.

$\mathrm{Hab}$. South Africa.

a. Purchased, April 23, 1879. 


\section{Order OPHIDIA.}

153. Python seba (Gm.). West-African Python. $H a b$. West Africa.

t. Presented by G. H. Garrett, Esq., June 13, 1879.

u. Deposited, June 21, 1879.

154. Python regius (Shaw). Royal Python.

Hab. West Africa.

m-o. Received in exchange, Oct. 8, 1879.

155. Python molurus (Linn.). Indian Python. $H a b$. India.

j. Presented by Mr. George Billett, July 7, 1879.

157. Python reticulatus (Schn.). Reticulated Python. Hab. Malacca.

b. Purchased, June 30, 1879.

160. Boa constrictor, Linn. Common Boa.

Hab. South America.

$u^{2}, v^{2}$. Deposited, April 2, 1879.

$w^{2}$. Purchased, May 3, 1879.

$x^{2}$. Presented by Dr. A. Stradling, July 1, 1879.

$y^{2}-a^{3}$. Presented by W. Young, Esq., Nov. 1, 1879.

163. Eunectes murinus (Linn.). Anaconda.

Hab. South America.

j. Received in exchange, April 2, 1879.

k. Presented by G. H. Hawtayne, Esq., May 1, 1879.

l. Presented by Capt. E. Ball, Oct. 1, 1879 .

m. Purchased, Nov. 15, 1879.

164. Epicrates anyulifer, Dum. \& Bibr. Pale-headed TreeBoa.

Hab. Cuba.

$i, j$. Hybrids between a male of this species and a female Chilobothrus inornatus. Born in the Menagerie, Sept. 9, 1879. 
166. Pelophilus madagascariensis, Dum. \& Bibr. Madagascar Boa.

Hab. Madagascar.

b. Presented by Canon G. Ogilvy, May 4, 1879.

*167a. Tortrix scytale, Oppell. Coral Snake.

Hab. Demerara.

a. Presented by G. H. Hawtayne, Esq., C.M.Z.S., Aug. 5, 1879. From Demerara.

*171a. Rhinostoma cupreum, Günther. Coppery Snake. Hab. Africa.

a. Purchased, April 23, 1879.

*173a. Rhinechis scalaris, Schinz. Back-marked Snake.

$H a b$. Southern Europe.

a. Purchased, June 9, 1879.

178. Coronella lavis, Lacép. Smooth Snake. Hab. Europe.

t. Purchased, July 16, 1879. From Hampshire.

u. Presented by - Dart, Esq., Aug. 21, 1879.

$v$. Born in the Menagerie, Nov. 21, 1879.

183. Liophis merremi (Max.). Merrem's Snake. Hab. Monte Video. $c, d$, e. Purchased, Jan. 16, 1879.

183a. Liophis cobella (Linn.). Cobella Snake. Hab. South America.

a. Presented by G. H. Hawtayne, Esq., C.M.Z.S., Aug. 5, 1879. From Demerara.

193. Tropidonotus leberis (Linn.). Seven-banded Snake. Hab. North America. o-t. Bred in the Gardens, May 9, 1879. 
204. Spilotes variabilis (Max.). South-American Rat-Snake. $H a b$. South America.

g. Presented by Mr. George Billett, July 7, 1879.

h. Presented by Thomas Horrod, Esq., Nov. 20, 1879.

*212a. Geoptyas collaris, Steindachner. Neck-marked Snake. Hab. Brazil.

a. Purchased, April 5, 1879.

218. Psammophis crucifer (Merr.). Crossed Snake. $H a b$. South Africa.

b. Purchased, April 23, 1879.

221. Colopeltis lacertina (Geoffr.). Lacertine Snake. $H a b$. South Europe and North Africa.

o. Presented by Commander J. Pratt, June 15, 1879.

p. Purchased, Oct. 7, 1879.

226. Leptodira annulata (Linn.). Annulated Snake. Hab. Panama.

e. Presented by R. T. Daris, Esq., Aug. 26, 1879.

228. Oxyrrhopus doliatus, Dum. \& Bibr. Square-spotted Snake.

$H a b$. South America.

b. Presented by H. Colgate, Esq., Sept. 15, 1879.

231. Boodon lineatus (Dum. \& Bibr.). Lineated Boodon. $\mathrm{Hab}$. South Africa.

b. Purchased, April 23, 1879.

239. Elaps lemniscatus (Linn.). Chequered Elaps. Hab. South America.

c. Deposited, April 2, 1879.

d. Presented by Dr. A. Stradling, Aug. 2†, 1879.

246. Vipera rhinoceros, Schl. River- Jack Viper.

Hab. West Africa.

$k-n$. Received in exchange, Jan. 28, 1879. 
247. Vipera arietans, Merrem. Puff-Adder. Hab. Cape of Good Hope.

w. Purchased, April 21, 1879.

$x$. Presented by Surgeon F. Speer, May 26, 1879.

y. Presented by the Rev. G. H. R. Fisk, C.M.Z.S., Dec. 15, 1879.

255. Crotalus horridus, Linn. Horrid Rattlesnake.

$H a b$. Nicaragua.

$c, d$. Purchased, Sept. 3, 1879. (Young.) 


\section{Class AMPHIBIA.}

\section{Order BA'TRACHIA.}

2. Bufo calamita, Laur. Natterjack Toad. Hab. Cornwall.

n. Presented by H. A. Macpherson, Esq., Oct. 6, 1879.

14. Bombinator igneus (Laur.). Fire-bellied Toad. Hab. Europe.

7c. Presented by H. A. Macpherson, Esq., Oct. 6, 1879.

18. Hyla arborea (Linn.). European Tree-Frog. Hab. Europe.

$g^{3}, n^{3}$. Presented by the Rev. S. R. Wilkinson, F.Z.S., Oct. 3 , 1879.

$0^{3}-r^{3}$. Presented by H. A. Macpherson, Esq., Oct. 6, 1879.

$s^{3}-x^{3}$. Presented by A. Leipner, Esq., F.Z.S., Oct. 8, 1879.

25. Pelodryas carulea (White). White's Tree-Frog.

Hab. New South Wales,

h, i. Purchased, Feb. 19, 1879.

\section{Order URODELA.}

26. Salamandra maculosa (Laur.). Spotted Salamander. Hab. Europe.

$m^{2}-f^{3}$. Purchased, June 16, 1879.

$g^{3}-q^{3}$. Purchased, June 18, 1879.

$r^{3}-t^{3}$. Presented by the Rev. S. R. Wilkinson, F.Z.S., Oct. 3, 1879.

39. Amphiuma means, Cuv. Amphiuma.

Hab. North America.

d. Presented by A. C. Cole, Esq., A pril 24, 18 `9. 


\section{Class PISCES.}

*11a. Huro nigricans, Cuv. Black Bass.

$H a b$. Fresh waters of N. America.

27. Anabas scandens (Dald.). Climbing Anabas.

$H a b$. India.

$c-g$. Presented by Dr. G. E. Dobson, C.M.Z.S., June 10, 1879.

49. Malapterurus beninensis, Murray. Electric Silurus.

Hab. West Africa.

c-e. Purchased, Aug. 27, 1879.

*58a. Fundulus majalis, Art. Amcrican Mayfish.

$H a b$. North America. 


\section{APPENDIX.}

The following species were accidentally omitted in the last Edition of the list :-

115a. Estrelda formosa (Latham). Green Waxbill.

$H a b$. India.

$a-$ e. Purchased, Feb. 1, 1878.

136a. Munia rubro-nigra, Hodgson. Chestnut-bellied Finch. $H a b$. India.

a. Presented by Miss M. Jackson, July 6, 1878.

156a. Euplectes nigroventris, Cassin. Black-chested Weaverbird.

Hab. East Africa.

a-c. Presented by A. Brown, Esq., July 11, 1878.

The following correction should be made, p. 258, Aves, no. 424:-Ramphastos tocard, Vieill., should be Ramphastos ambiguus, Sw. 



\section{N D E X.}

Page

Accentor, Alpine...... 21 Accentor collaris ...... 21 Acontias melcagris ... 58 Acouchy .............. 14 Acridotheres cristatel-

lus. 26

Adder, Puff .......... 62 Egialitis hiaticula ... 52 Agouti, Central-

American ............ 13 -, Hairy-rumped. 14 Alauda arvensis ..... 26 Alcedo ispida ........ 28 Alligator ............. 56 Alligator latirostris... 57 - mississippiensis. 56 Amazon,Blue-cheeked 33 - Blue-faced ...... 33 —, Bodinus's ...... 33

—, Dufresne's ...... 33 —, Golden-naped . 34 —, Guilding's...... 33 —, Red-tailed ...... 33 - Yellow-fronted. 34 Ammoperdix heyi...... 47 Ampelis garrula ...... 22 Amphisbana darwini. 57 Amphiuma ........... 63 Amphiuma means ... 63 Inabas, Climbing ...6 64 Anabas scandens ...... 64 Inaconda .............. 59 Anas boschas.......... 42

- castanea ........ 43

- pecilorhyncha... 43

— strepera ........ 43

_ superciliosa ...... 43

- xanthorhyncha . 43

Anguis fragilis........ 58

Anous stolidus ......... 54

Anser ferus ........... 41

- segetum ......... 41

Ant-bear, Cape ..... 19
Page

Ant-eater, Tamandua 19

— Indian ........ 16

—, Isabelline ...... 16

- Pronghorn...... 16

Antilocapra ameri-

cana ................ 16

Antilope cervicapra ... 16

Aprosmictus scupula-

tus 31

Aptenodytes pennanti. 55 Aquila adalberti ...... 36

— chrysaetos ...... 36

- næevioides......... 36

Ara ararauna ......... 32

chloroptera ...... 32 macio ........... 32

Archibuteo lagopus ... 35 Arctomys caudatus ... 12

$-\operatorname{momax} . . . . . . . . . .12$

Ardea egretta ........ 39

- russata............ 39

Ardetta involucris ... 39

Argus giganterıs ...... 49

Armadillo, Long-

haired .............. 19

$\longrightarrow$, Peba ........... 18

Astur approximans ... 37

Ateles ater.............. 4

- geoffroyi ......... 4

Atherura africana ... 13

Axis galericulata...... 45

- sponsa .......... 44

Baboon, Chacma..... 4

-, Guinea ......... 4

$\longrightarrow$, Yellow ......... 4

Badger, Common ... 10

Balearica pavinina... 52

—_regulorum ...... 52

Barn-Owl, Common . 34

Jass, Black ........... 6t

Bat, Collared Fruit... 7 \begin{tabular}{lrr} 
Bat, Indian Fruit ... & 7 \\
, Long-eared...... & 7 \\
Bear, Black ............ & 11 \\
\hline , Brown ......... & 11 \\
\hline , Syrian............ & 11
\end{tabular}

Beaver, Canadian ... 12

Beech-ILarten ........ 10

Belideus sciureus ...... 19

Bell-bird, Naked-

throated ........... 27

Bernicla brenta .....41

- canadensis ..... 41

- jubata ........... 41

_ leucopsis .......... 41

- magellanica...... 41

_- poliocephala ... 42

Bittern, Variegated... 39

Black Bass ........... 64

Boa, Common ......... 59

-, Madagascar ... 66

- Pale-headed

Tree ................ 59

Boa constrictor......... 59

Boatbill ............. 39

Bolborhynchus mona-

chus ................ 33

Bombinator igneus ... 63

Boodon, Lineated ... 61

Boodon lineatus ...... 61

Bos indicus ........... 15

Bower-bird, Silky ... 26

Brachyurus rubicun-

dus ............... 5

Broadtail, Adelaide 31

-, Rose-Hill ...... 31

-, Yellow-bellied . 32

Brotogerys tiriacula... 34

- tori .............. 3t

Tuipara ......... 3t

Bubo maculosus ..... 35

- maximus ......... 34

Bubalus cafter ......... 15

Euccros atratus ...... $: 8$ 
Page
Buceros subcylindricus 28

Buffalo, Cape

Bufo calamita ........ 15

Bulbul, Blue-winged

Green .............. 22

Bullfinch, Common... 25

Bunting, Common ... 25

$\longrightarrow$, Reed ............ 25

$\longrightarrow$, Yellow ......... 25

Bush-Dog............. 10

Bustard, Great ..... 51

- Houbara ...... 51

Buteo borealis ......... 35

— vulgaris ........ 35

Buzzard, Common ... 35

-, Northern ..... 35

$\longrightarrow$, Rough-legged . 35

Cacatua galerita ...... 2y

- moluccensis ...... 29

- ophthalmica .... 29

— roseicapilla ...... 30

- sulphurea......... 30

Callipepla californica 47

Calliste fastuosa ..... 22

- melanonota ...... 22

Calonas nicobarica ... 46

Calyptorhynchus

banksii .............. 30

funereus .......... 30

Camel, Bactrian ...... 15

Camelopardalisgiraffa 17

Camelus bactrianus ... 15

Cancroma cochlearia . 39

Canis aureus ........ 9

- fulvus ............ 9

— mesomelas ...... 9

- rudis ............. 9

Capreolus pygargus ... 18

Capricornis crispus ... 16

Capuchin, Weeper ... 5

- White-cheeked. 4

- White-fronted . 5

- White-tliroated 5

Capybara ............. 14

Caracal................ 8

Cardinal, Black-

crested .............. 23

Cardinalis virginianus -24

Carduelis elegans ...... 24

Cariacus macrotis ... 18

- mexicanus ...... 18

- rufus ............ 18

Cariama, Brazilian ... 51

Cariama cristata ...... 51

Castor canadensis...... 12

Cat, African Civet ... 8 , Caffer Wild ... 8

—, Geoffroy's ...... 8
Page

Catoblepas gnu......... 16

Cavy, Rock ........... 14

Cayman, Broad-

snouted............... 57

Cebus albifrons......... 5

- - capucinus ...... 5

- hypoleucus ...... 5

Cephalophus rufilatus 16

Cercocebus albigena... 2

Cercoleptes caudivol-

vulus.................. 11

Cercopithecus callitrichus

- cephus ..............
- yynosurus ......
- diana ..........
griseo-viridis ...
- malandii .........
- petaurista........

Cerodon rupestris...... 14

Cervicapra isabellina. 16

Cervulus reevesi ...... 17

Cervus aristotelis ...... 17

- axis ............... 17

elaphus ......... 17

sika .............. 17

Chaffinch, Common . 24

Chamaleon vulgaris . 57

Chamcepelia passerina 46

Chameleon, Common 57

Chasmorhynchus nu-

dicollis .............. 27

Chauna chavaria ...... 40

Cheetah ............. 7

Chenalopex regyptiaca 40

jubata ........... 41

Chilobothrus inorna-

tus .................... 59

Chinchilla ........... 13

Chinchilla lanigera ... 13

Chionis alba ........... 53

Chrysomitris spinus... 24

Chrysothrix sciurea... 5

Chrysotis auripalliata 34

bodini ............ 33

- bouqueti ........... 33

— coligena ......... 33

_ dufresniana...... 33

_ erythrura........ 33

- guildingi.......... 33

__ ochrocephala ... 34

Ciconia alba........... 39

nigra ............ 39

Cinclus aquaticus...... 21

Clemmys ornata ...... 56

- punctularia ...... 56

Coati, Ring-tailed ... 11

Cockatoo, Banksian ... 30
Page

Cockatoo, Blue-eyed.. 29

, Funereal......... 30

- -, Greater Sul-

phur-crested ..... 29

- Lesser Sulphur-

crested

$\longrightarrow$, Roseate .......... 30

—. Rose-crested ... 29

, Slender-billed... 30

Cock-of-the-rock, $\mathrm{De}$ -

meraran ........... 27

Colopeltis lacertina ... 61

Coreba cyanea ........ 22

Colin, Crested......... 47

Colobus bicolor......... 1

Columba guinea ....... 45

speciosa ........ 45

Colymbus septentrio-

nalis ................. 55

Conepatus mapurito... 10

Conure, Golden-

headed ............... 33

Conurus auricapillus. . 33

Coronella lavis........ 60

Coryphospingus pilea-

tus .................... 24

Corythaix porphyreo-

lopus .................. 28

Coypu ............... 13

Crane, Balearic ...... 52

$\longrightarrow$, Cape Crowned. 52

-, Stanley ........ 52

Crax globicera ......... 50 sclateri............ 50

Crossbill, Common ... 25

Crotalus horridus...... 62

Crotaphytus wisli-

zenii ................. 58

Crow, White-backed

Piping ............. 27

Crypturus noctivagus. 55

Cuckoo, Common. ... 29

Cuculus canorus ...... 29

Curassow, Globose ... 50 , Sclater's ........ 50

Curlew, Common...... 53

Cyanocitta cristata ... 27

Cyanocorax cyanome-

las.................... 27

- cyanopogon ...... 27

- pileatus ........ 27

Cyanorhamphus nove-

zealandice ........... 32

Cygnus atratus......... 42

nigricollis........ 42

Cynocephalus babouin 4

porcarius ......... 4

- sphin $x \ldots . . . . . . .4$

Cynomys ludovicianus 12 
Page
Cynonycteris collaris . 7

Dacelo gigantea 28

Dafila acuta ............ 44 - spinicauda ...... 44 Dasyprocta acouchy... 14 _ isthmica ......... 13 $\begin{array}{llll}\text { prymnolopha } & \text {... } & 14\end{array}$

Dasypus vellerosus ... 19

Deer, Axis ........... 17

-, Chinese Water 18 , Japanese......... 17 , Mexican ......... 18 , Michie's Tufted 17

, Mule ........... 18

, Red.............. 17

, Red Brocket ... 18 Sambur ......... 17

, White-rumped

Red .................. 18

Dendrocitta sinensis... 27

Dendrocygna autumnalis

viduata.......... 42

Dicotyles tajaçu ...... 15

Didelphys cancrivora.. 19

Diuca grisea ............ 23

Diver, Red-throated .. 55

Dog, Bush .......... 10

Dormouse, Fat $\quad$...... 12

Dove, Barred ......... 45

—, Geoffroy's ....... 46

—, Martinican...... 46

—, Passerine ...... 46

—-, Stair's Ground. 46

Duck, Australian..... 43

- Chestnut-

breasted

—, Common ...... 42

—, Gadwall .......... 43

, Mandarin ...... 45

—, Red-billed Tree 42

—, Rosy.billed ... 45

—, Spotted-billed .. 43

_-, Summer ........ 44

— Tufted ......... 45

_ White - faced

Tree ................... 42

, Yellow-billed... 43

Dunlin ................. 53

Eagle, Bateleur ...... 36

—, Bonelli's......... 36

$\longrightarrow$ Booted ......... 36

—, Chilian Sea ... 35

—, Golden ......... 36

—, Harpy .......... 35

—, Mace's Sea ...... 36

_, Spanish Im-

perial 36
Prge

Wagle, Tawny ......... 36

-_, Vociferous ...... 36

Egret, Buff-backed ... 39

-, Great American 39

Eland

Elaphodus michianus. 17

Elaps, Chequered ...6 61

Elaps lemniscatus ... 61

Emberiza citrinella ... 25

- miliaria .......... 25

schoriclies ....... 25

Epicrates angulifer ... 59

Eremophila chryso-

lama ................ 26

Estrelda cinerea ...... 23

formosa ......... 6.5

Endocimus albus ...... 40

— ruber ............. 40

Eunectes murinus...... 59

Euphema elegans ...... 31

- pulchella ......... 31

Euphonia crassirostris 22

_ violacea ........ 22

Euplectes nigroventris .................6 65

Euplocamus nycteme-

rus.................... 48

- prcelatus ......... 48

Eupsychortyx cristatus ................. 47

Europyga helias ...... 52

Falco barbarus ........ 37

— lanarius ......... 37 - peregrinus ...... 37

Falcon, Barbary ...... 37

_- Lanner ......... 37

_—, Laughing ...... 38

- , Peregrine ...... 37

Felis caffra ............ 8

- caracal............ 8

- concolor ........... 8

— geoffroii ......... 8

— jubata ............. 7

- pardalis .......... 8

— pardus............. 7

—_igris ............ 7

Finch, Bluish ........ 24

, Chestnut-

bellied

65

_-, Diuca .............. 23

—, Melodious ...... 23

-, Mountain ....... 24

_-, Pileated .......... 24

—, Saffron ......... 24

, Wiener's........... 23

Flamingo, South-

American ............ 40

Fox, Common .......... 9

$\longrightarrow$ Red.............. 9
Fox, Rude ........... Page

Fregata aquila.......... 38

Frigate-bird, Great... 38

Fringilla coelebs ...... 21

—_ nontifringilla ... 24

Frog, European Tree 63

- White's Tree ... 6:3

Fuligula cristata ...... 4.5

- ferina ........... 4.5

Fundulus majalis..... 6t

Galago, Garnett's ... 7

$\longrightarrow$ Grand............. 6

Galago crassicaudata 6

—_ garnetti ........ 7

Galictis barbara ...... 10

Gallus varius ......... 48

Gannet ................. 38

Garrulus lanceolatus. 27

Gazella arabica ..... 16

- dorcas ............ 15

sœmmerringi ... 16

_ subgutturosa $\ldots . .16$

Gazello................ 15

$\longrightarrow$, Arabian ......... 16

, Persian ......... 16

—, Scmmerring'... 16

Gecko, European...... 57

Geopelia striata ..... 45

Geoptyas collaris ...... 61

Geranoaetus melano-

leucus ............... 35

Gerbillus indicus ...... 13

Giraffe ............... 17

Gnu, White-tailed ... 16

Goat-Antelope, Japa-

nese ................ 16

Godwit, Bar-tailed ... 53

- , Black-tailed ... 53

Goldfinch .............. 24

Goose, Ashy-headed .. 42

$\longrightarrow$, Bean ........... 41

—, Bernicle ........ 41

—, Brent........... 41

—, Canada ......... 41

—, Egyptian ..... 41

$\longrightarrow$, Maned ......... 41

—, Orinoco ......... 41

—, Upland .......... 41

, Wild or Grey-

lag.................. 41

Goshawk, Allied ...... 37

Goura coronata ...... 46

- victoria ......... 47

Gracula religiosa..... 26

Grass-Parrakeet, Ele-

gant ............... 31

—, Turquoisine ... 31 , Undulated ...... 31

Grebe, Little ........ 55 
Page

Greenfinch, Common 24 Grosbeak, Brazilian Blue ................ 23

—_, Cardinal........ 24

Gubernatrix cristaiella 23

Guinea-fowl, Abyssinian

—, Crested .......... 49

_., Elliot's ......... 49

—, Mitred .......... 49

__, Rendall's ..... 49

__, Vulturine ....... 50

Guiraca cyanea ...... 23

Gull, Black-headed... 54

—, Common ...... 54

_-, Herring ......... 5t

__, Jameson's ...... 54

__, Lesser Black-

backed .............. 54

__, Yellow-legged

Herring ........... 54

Gymnorhina leuconota 27

Gypaetus barbatus ... 38

Haliaetus leucoryphus 36 vocifer ............ 36

Halmaturus bennettii . 20

- brachyurus ..... 20

Hapale jacchus........ 5

- penicillata ...... 5

Hare, Brazilian ...... 14

-, Common ...... 14

Hawk, Many-zoned... 36

Helotarsus ecaudatus . 36

Herpestes auropunctatus ..................

- fasciatus .......... 9

—_ griseus ........... 9

Herpetotheres cachin. nans ................. 38

Himantopus nigricollis ................. 53

Hog, Red-River ...... 15

Hornbill, Black ...... 28

_-, Subcylindrical . 28

Houbara undulata ... 51

Huro nigricans ..... 64

Hyæna, Brown ..... 8

-, Striped ........ 8

Hycna brunnea ..... 8

- striata .......... 8

Hydrochorus capybara ................. 14

Hydropotes inermis ... 19

Hyla arborea ........6 63

Hypsiprymus gai-

nardi .............. 20

Hyrax, Cape........... 14

Hyrax capensis ..... 14
Hystrix cristata ..... 13

Ibis, Black-faced

40

- Scarlet

40

—, White............ 40

Ichneumon, Banded.. 9

—, Grey ............ 9

- , Spotted ......... 9

Icticyon venaticus ... 10

$\begin{array}{llll}\text { Iguana tuberculata } & . . . & 57\end{array}$

Iguana, Tuberculated 57

Jackal, Black-backed 9

—, Common ...... 9

Jay, Black-headed ... 27

_-, Blue ........... 27

__, Blue-bearded.... 27

, Pileated ......... 27

Jungle-fowl, Fork-

tailed

Kangaroo, Blackfaced ................. 20

$\longrightarrow$, Gainard's Rat.. 20

$\longrightarrow$ Great ............ 19

—

, Yellow-footed

Kestrel, American ... 37

$\longrightarrow$, Common......... 37

Ketupa javanensis ... 35

Kingfisher ........... 28

, Laughing ...... 28

Kinkajou ............... 11

Kite, Egyptian......... 37

$\longrightarrow$, Indian ......... 37

Knot................... 53

Lacerta viridis........ 58

Lapwing, Cayenne ... 52

Lark, Sky............. 26

—, White-bellied... 26

Larus argentatus ...... 54

- cachinnans ....... 54

- canus ............. 54

- fuscus .............. 54

__ nova-hollandia. 54

—_ ridibundus ...... 54

Lemming, Norwegian 13

Lemur cutta ............ 6

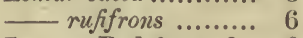

Leunur, Red-fronted .. 6 $\longrightarrow$, Ring-tailed ... 6 Leopard ............... 7 Leptodira anmulata ... 61 Lepus brasiliensis...... 14

- europaus......... 14

Licmetis tenuirostris . 30

Iigurinus chlorls ...... 24

Limosa lapponica...... 53
Page

Limosa melanura...... 53

Linnet, Comınon...... 25

Linota cannabina...... 25

- rufescens ......... 25

Liophis cobevla ......... 60

- merremi .........6 60

Lizard, Green ......... 58

-, Horned ......... 58

—

$\longrightarrow$, Tuatera .......... 57

—_, Wizlezen's ....... 58

Lophophorus impey-

anus ................. 47

Loris gracilis ......... 6

Loris, Slender ......... 6

$\longrightarrow$, Slow ........... 6

Lorius garrulus ...... 30

Lory, Chattering...... 30

Loxia curvirostra...... 25

Lutra nair ........... 10

Macacus cynomolgus.. 3

- erythrceus......... 3

— nemestrinus...... 3

- radiatus ......... 3

Macaw, Biue-and-

yellow ............. 32

$\longrightarrow$, Red-and-blue... 32

, Red-and-yellow 32

Macropus giganteus... 19

— melanops ......... 20

- rufus ........... 20

Magpie, Common ... 26

Maize-eater, Dark

Green .............. 26

Malapterurus beni-

nensis .............6 64

Mareca chiloensis...... 44

- penelope ......... 44

Marmoset,Black-eared 5

- , Conimon ...... 5

$\longrightarrow$, Geoffroy's ...... 5

$\longrightarrow$, Silky .......... 6

Marmot, Long-tailed. 12

$\longrightarrow$, Prairie ......... 12

$\longrightarrow$ Quebec .......... 12

Marten, Beech........ 10

Martes foina............ 10

Mastigure, Small-

scaled .............. 57

Mayfish, American ... 64

Meleagris gallo-paro.. 49

Meles taxus ........... 10

Melierax polyzonus ... 36

Melopsittacus undu-

latus ................. 31

Metopiana peposaca... 45

Midas geoffroii........ 5

— rosalia ............. 6

— ursulus............. 5 
Milvus agyptius ...... 37

-govinda ........ 37

Monnul, Himalayan .. 47

Monkey, Black-faced Spider

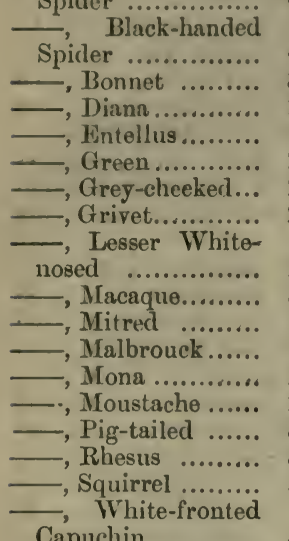

Capuchin ............

,White-thighed.. 1

, Vervet ....... 1

Motacilla yarrellii ... 21

Munia rubro-vigra ... 65

Muntjac, Reeves's ... 17

Mus rattus ............ 13

Mustela vulgaris ...... 10

Mynah, Chinese ...... 26

-, Malabar........ 26

$\longrightarrow$, Small Hill ...... 26

Myodes lemmus ...... 13

Myopotamus coypus... 13

Myoxus glis ............ 12

Nasua rufa ........... 11

Natterjack Toad ...... 63

Night-Heron, Common 39

- Violaceous...... 39

Nisaetus fasciatus...... 36

- pennatus ........ 36

Numenius arquata ... 53

- pheopus ......... 53

Numida oristata ..... 49

- ellioti ............ 49

- mitrata .......... 49

— ptilorhyncha ... 49

- rendalii ......... 49

- vulturina.......... 50

Nuthatch, Common... 21

Nycticebus tardigradus 6

Nycticorax griseus ... 39

- violaceus ........ 39

Nymphicus cornutus. . 31

Ocelot
Octodon, Cuming's ... 13 Octodon cumingi ...... 13 Ocyphaps lophotes ... 46 Ododontophorus guia-

nensis ............ 47

Opossum, Crab-eating 19

Oreas canna ............ 15

Orycteropus capensis.. 19

Otaria jubata ......... 11

Otis tarda.............. 51

Otter, Indian ......... 10

Ouaran, Caspian ...... 58

Ouzel, Water ......... 21

Ovis aries............. 17

Owl, Common Barn . 34

$\longrightarrow$, Downy ......... 35

- European Scops 35

—, Great Eagle ... 34

- Javan ........... 35

-, Spotted Eagle.. 35

-, Wood ............ 34

Oxyrrhopus doliatus. . 61

Palcornis docilis ...... 30

+ ja vanicus......... 30 torquatus.......... 30

Paradoxure, Common 8

- White-whiskered 9

Paradoxurus leuco-

mystax ............. 9

typus .......... 8

Parrakeet, All-green . 34

$\longrightarrow$ Beautiful ...... 32

—, Black-tailed .... 31

—, Elegant Grass.. 31 , Golden-fronted. 34

—, Grey-breasted. . 33

$\longrightarrow$, Horned ........ 31

-, Jaran ........... 30

—, King ............ 31

—, Masked ........ 32

-, New-Zealand... 32

—, Ring-necked ... 30

—, Rose-ringed ... 30

—, Tabuan ......... 32

$\longrightarrow$, Tovi ............ 34

—, Turqoisine...... 31

- Undulated

Grass ............. 31

Partridge, Guiana .... 47

$\longrightarrow$, Hey's ............ 47

Pavo cristatus ......... 48

- nigripennis ...... 48

- spicifer............ 48

Peafowl, Common .... 48

-, Black-winged... 48

—, Javan ............ 48

Peccary, Collared .... 15

Pelecanus mitratus ... 38

Pelican, Mitred ..... 38
Pago

Pelodryas carulea ... 63 Pelophilus madagasca-

riensis ..............6 60

Penguin, Black-footed 55

—, King ........... 55

Peristera geoffroii ... 4t

Perodicticus potto...... $\quad$ 6

Petrogale xanthopus... 20

Phalanger, Squirrel-

like ................. 19

- , Vulpine ........... 19

Phalangista vulpina . 19

Phasianus wallichii... 47

Pheasant, Amherst's . 48

$\longrightarrow$, Argus ............ 49

—, Cheer ............. 47

—, Gold ........... 47

—, Peacock ......... 49

—, Siamese ..........4 48

$\longrightarrow$, Silver ............. 48

Phlogæenas stairi ....... 46

Phoca vitulina......... 11

Phrnicopterus igni-

palliatus ............ 40

Phonipara canora ... 23

Phrynosoma cornutum 58

Phyllodactylus euro-

peeus ................ 57

Phyllornis hardwickii 22

Pica rustica ............ 26

Picus major ............. 28

Pigeon, Common

Crowned ............ 46

- , Crested .......... 46

- Nicobar ......... 46

$\longrightarrow$ Specious.......... 45

___, Triangular-

spotted ............. 45

$\longrightarrow$, Victoria Crowned 47

Pintail, Chilian ...... 44

$\longrightarrow$, Common ...... 44

Platalea lencorodia ... 40

Platycercus adelaidos. 31

- eximius ......... 31

- flaviventris ..... 32

Plecotus auritus ...... 7

Plover, Grey ........ 52

—, Ringed ........ 52

Pochard, Red-headed 45

Podargus, Cuvier's ... 28

Podargus cuvieri ...... 28

Podiceps minor......... 55

Poëphagus grunniens. 15

Polyplectron chinquis. 49

Polytelis melanura ... 31

Porcupine, African

Brush-triled........ 13

$\longrightarrow$, Crested ........... 13

Porphyrio, Allen's ... 51

-, Madagasear ... 51 


\section{Page}

Porphyrio alleni ...... 51 madagascariensis 51 martinicus ...... 51

Potamochœrus penicil-

latus ................. 15

Potto, Bosman's ...... 6

Procyon cancrivorus. . 10

Psammophis crucifer . 61

Psammosaurus caspicus 58

Psephotus pulcherrimus 32

Pseudoleistes virescens. 26

Psophia crepitans ... 51

- viridis ........... 51

Pteropus medius ...... 7

Ptilonorhynchus violaceus

Pucras, Darwin's...... 48

Pucrasia darwini..... 48

Puff-Adder ............ 62

Pulsatrix torquata ... 35

Puma ................. 8

Pyranga saira........ 23

Pyrrhula europaa ... 25

Pyrrhulopsis personata 32 tabuensis

Pytelia wieneri......... 23

Python, Indian ...... 59

- Reticulated ... 59

—, Royal............ 59

—, West-African... 59

Python molurus ...... 59

regius ............. 59

- reticulatus ...... 59

- seba.............. 59

Quail, Californian ... 47 Querquedula brasili-

ensis

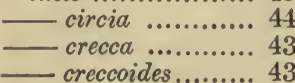

Raccoon, Crab-eating. 10

Rail, Bar-winged...... 50

$\longrightarrow$, Sooty ............ 50

-, Water............. 50

-, White-spotted . 50

Rallus aquaticus ...... 50

- maculatus ...... 50

- pciciloptera ....... 50

- rhythirhynchus, . 50

Ramphastos ariel ...... 29

- carinatus......... 29

Ramphocolus brasilius 23

Rat, Black ........... 13

- Indian Jerboa.. 13

Rattlesnake, Horrid. . 62

Redpole, Lesser ...... 25

Reed-Bunting ......... 25

Rhea americana ..... 55
Page

Rhea, Common ...... 55

Rhinechis scalaris ... 60

Rhinoceros, Sumatran 14

Rhinoceros sumatrensis 14

Rhinostoma cupreum . 60

Robin, American...... 21

Rock-Cavy ............ 14

Rupicola crocea ...... 27

Saki, Red-faced ...... 5

Salamander, Spotted . 63

Salamandra maculosa 63

Sciuropterus fimbriatus 12

Sciurus plantani ...... 12

— vulpinus ........ 12

- vulpinus, var.

capistrata ........... 12

Scolopax rusticula ... 53

Scops giu ............. 35

Screamer, Crested ... 40

Seal, Common ......... 11

Sea-Lion, Yatagonian 11

Selenidera maculiros-

tris

Semnopithecus entellus 1

- mitratus ......... 1

Serpentarius reptilivorus.

Sheathbill, Yellowbilled 38

58

Sheep, Domestic ...... 17

Sheldrake, Common. . 42

-, Ruddy ........ 42

Shoveller ............... 44

Silurus, Electric ...... 64

Siskin, Common ...... 24

Sitta casia ............ 21

Skua, Pomatorhine... 54

Skunk, Chilian......... 10

Sky-Lark ............. 26

Slowworm ........... 58

$\longrightarrow$, Spotted .......... 58

Snake, Annulated ... 61

—, Back-marked... 60

$\longrightarrow$, Cobella ......... 60

—, Coppery......... 60

- Coral ............ 60

- Crossed ......... 61

-

__, Merrem's ...... 60

—, Neck-marked ... 61

- Seven-banded... 60

—, Smooth ........ 60

-, South-American 61

_, Square-spotted. 61

Song-Sparrow,Pileated 25

Sparrow,Pileated Song 25

Spatula clypeata ..... 44

Spermophila carules-

cens ................. 2
Page

Spheniscus demersus.. 55

Sphenodon punctatus . 57

Spilotes variabilis ... 61

Spoonbill, Common... 40

Squatarola helvetica... 52

Squirrel, Grey Flying 12

$\longrightarrow$, Plantain......... 12

—, Vulpine ......... 12

Starling, Common ... 26

Stercorarius pomato-

rhinus .............. 54

Stilt, Black-necked ... 53

Stork, Black........... 39

—, White............ 39

Strepsilas interpres ... 52

Strix flammea ........ 34

Sturnia malabarica ... 26

Sturnus vulgaris ...... 26

Sugar-bird, Yellow-

winged ............. 22

Sula bassana ........ 38

Sun-Bittern ........... 52

Sus leucomystax ...... 15

Swan, Black .......... 42

—, Black-necked ... 42

Swine, White-whis-

kered................. 15

Sycalis flaveola ...... 24

Syrnium aluco ........ 34

Tachyphonuscoronatus 22

Tadorna rutila........ 42

- vulpanser.......... 42

Tamandua tetradactyla 19

Tamarin, Negro ...... 5

Tanager, Black-shoul-

dered .............. 22

$\longrightarrow$, Brazilian ...... 23

-, Crowned......... 22

—, Palm ............ 22

—, Saira ........... 23

- Superb .......... 22

— Thick-billed ... 22

, Violet........... 22

Tanagra palmarum ... 22

Tantalus, American. . 40

Tantalus loculator ... 40

Taraguira smithi...... 58

Tatusia peba............ 18

Tayra ................ 10

Teal, Brazilian......... 43

, Chilian ......... 43

—, Common ......4 43

—, Garganey ...... 44

Teguexin, Common... 58

Teius teguexin ......... 58

Tern, Noddy ........ 54

Terrapin, Adorned ... 56

-, Rough ........ 56

Testudo carbonaria ... 56 


\begin{tabular}{|c|c|}
\hline & Page \\
\hline estudo elephantina... 56 & Tringa canutus ... \\
\hline $\begin{array}{l}\text { - pardalis ......... } 56 \\
\text { radiata } \\
\end{array}$ & $\begin{array}{l}\text { cinclus........... } 53 \\
\text { Tropidonotus leberis... } 60\end{array}$ \\
\hline Tetrapteryx paradisea 52 & Trumpeter, Common 51 \\
\hline Thaumalia amherstice 48 & $\longrightarrow$, Green-winged .. 51 \\
\hline - picta ........... 47 & Turdus albiventris ... 21 \\
\hline Theristicus caudatus.. 40 & - migratorius...... 21 \\
\hline Thrasactus harpyia... 35 & - musicus .... \\
\hline $\begin{array}{r}\text { Thrush, Song } \ldots \ldots . . . .21 \\
\text { White-bellied... } 21\end{array}$ & $\begin{array}{l}\text { Turkey, North- } \\
\text { rican ........... }\end{array}$ \\
\hline iger................... 7 & Turnstone.... \\
\hline namou, Banded ... 55 & tledove, Chinese. . \\
\hline $\begin{array}{l}\text { Tinnunculus alauda- } \\
\quad \text { rius ................. } 37\end{array}$ & Turtur chinensis.. \\
\hline - sparverius ...... 37 & Uromastix microlepis \\
\hline $\begin{aligned} \text { Toad, Fire-bellied ... } & 63 \\
\text { Natterjack ...... } & 63\end{aligned}$ & us americanus ... \\
\hline Tortoise, Black ...... 56 & syriacus... \\
\hline $\begin{array}{l}\text { - Elephantine ... } 56 \\
\text {, Leopard ......... } 56\end{array}$ & yenennsis.. 52 \\
\hline _, Radiated........ 56 & Vidua principalis ... \\
\hline Tortrix scytale........ 60 & Viper, River Jack ... 61 \\
\hline Toucan, Ariel ... & Vipera arietans $\quad \ldots . .662$ \\
\hline -, Spotted .........29 29 & $\overline{\text { Viverra civetta.......... }}$ \\
\hline $\begin{array}{l}\text { Touracou, } \\
\text { crested }\end{array}$ & $\begin{array}{r}\text { Vultur auricularis ... } \\
- \text { monachus........ }\end{array}$ \\
\hline Po & ulture, Cinereous ... \\
\hline & \\
\hline
\end{tabular}

$\begin{array}{r}\text { Page } \\ \text { Vulture, Secretary } \ldots . .38 \\ \hline\end{array}$

Wagtail, Pied ........ 21

Wallaby, Bennett's... 20

-, Short-tailed ... 20

Waterhen, Martinique 51

Water-Ouzel ......... 21

Waxbill, Common ... 23

$\longrightarrow$, Green ............ 65

Waxwing ............. 23

Weasel .................. 10

Weaver-bird, Blackchested

Whimbrel ........... 53

Whydah-bird, Pintailed. ............... 23

Wigeon .............. 44

-, Chiloe........... 44

Woodcock ............ 53

Woodpecker, Greater Spotted............. 28

Yak ................ 15

Zebu ................... 15

Zenaida martinicana. 46 Zonotrichia pileata ... 25

THE END.

Printed by TAylor and Francis, Red Lion Court, Fleet Street. 





Z
Z

\section{3}

Zoological Society of

London

Revised list of the vertebrated arimals

Supplement, 187\%, 1873, 1874 and 1879.

PLEASE DO NOT REMOVE CARDS OR SLIPS FROM THIS POCKET

\section{UNIVERSITY OF TORONTO LIBRARY}


